\title{
GLOBAL EXISTENCE OF SOLUTIONS BY PATH DECOMPOSITION FOR A MODEL OF MULTIPHASE FLOW
}

\author{
BY \\ FUMIOKI ASAKURA (Department of Asset Management, Osaka Electro-Communication \\ University, Neyagawa, Osaka, Japan) \\ AND \\ ANDREA CORLI (Department of Mathematics, University of Ferrara, Ferrara, Italy)
}

\begin{abstract}
We consider a strictly hyperbolic system of three conservation laws, in one space dimension. The system is a simple model for a fluid flow undergoing liquid-vapor phase transitions. We prove, by a front-tracking algorithm, that weak solutions exist for all times under a condition on the (large) variation of the initial data. An original issue is the control of interactions by means of decompositions of shock waves into paths.
\end{abstract}

1. Introduction. We consider a strictly hyperbolic system of conservation laws arising in modeling phase transitions for fluids, in one spatial dimension. In Lagrangian coordinates it is written as

$$
\begin{cases}v_{t}-u_{x} & =0 \\ u_{t}+p(v, \lambda)_{x} & =0 \\ \lambda_{t} & =0 .\end{cases}
$$

Here $v$ is the specific volume, $u$ the velocity and $\lambda$ the mass density fraction of the vapor in the fluid; the model is closed by the equation of state $p=a^{2}(\lambda) / v$ for the pressure, where $a$ is a positive increasing function. In this paper we are concerned with the global existence of weak solutions to the initial-value problem for (1.1), in the case of initial data with large total variation.

System (1.1) is derived by a more general model proposed and studied in [10, which includes reaction terms, viscosity, diffusion of the species and relaxation. The simple model (1.1) has many interesting properties that make it similar to the Nishida isothermal system [18 and to both the systems of isentropic [19] and nonisentropic gas dynamics [15], 16. Analogous systems have been considered in [20] and [14, for a two-mode reacting gas; see also [12, 13, for a model with a $\gamma$-law for the pressure, with varying $\gamma>1$. A related $2 \times 2$ system was studied in [8]. The existence of weak solutions to system (1.1) has been considered in the framework of compensated compactness theory,

Received April 28, 2011.

2010 Mathematics Subject Classification. Primary 35L65; Secondary 35D30, 76T30. 
[6], [1], [17, but either for different equations of state or for different assumptions on the data.

The global existence of weak solutions to system (1.1) for initial data with large total variation was first proved in [2] with a front-tracking scheme inspired by [4]. Preliminary results on the Riemann problem and interactions of waves have been given in [1]; there, the conservative quantities were used throughout. We refer to [3] for related results on a system with reactive source term.

In this paper we are concerned with the same issues of [2], but a rather different viewpoint allows us to learn more about the interaction of the large amplitude waves. In the interaction of small amplitude waves, the amplitude of the generated wave propagating in the $i$-characteristic direction is the linear superposition of the $i$-incoming waves plus a quadratic part. On the other hand, the linear part of the cancelation caused by the interaction of a shock wave and a rarefaction wave of large amplitudes is not well approximated by the superposition (see [19]).

We exploit a new front-tracking algorithm introduced in [5] and inspired by [22]. The main idea of this algorithm consists in decomposing the shock waves appearing in the approximate solution into primary and secondary paths, which take into account the linear and quadratic part of the interactions, respectively. This path-decomposition method enables us to give a geometric representation of the constraints to the initial data and also leads to study the asymptotic stability of the solution with large initial data (see [5]). Thus, this paper and [2] offer two complementary points of view on the same problem. Both are motivated by the lack of results on the global existence of weak solutions to systems of conservation laws for large data, apart from the cases of $2 \times 2$ systems or systems in the Temple class; system (1.1) belongs to neither of these classes.

The main result concerns the Cauchy problem for the system (1.1) with initial data $(\bar{v}, \bar{u}, \bar{\lambda})$ and is stated by using the notions of weighted total wariation $\operatorname{WTV}(a(\bar{\lambda}))$ of $a(\bar{\lambda})$ and total variation $\operatorname{TV}(\bar{v}, \bar{u})$ of $(\bar{v}, \bar{u})$ (see Section 2 for definitions). Under the constraint (2.6) for $\operatorname{WTV}(a(\bar{\lambda}))$, we prove that a global-in-time (weak) solution exists provided the product $\operatorname{WTV}(a(\bar{\lambda})) \cdot \operatorname{TV}(\bar{v}, \bar{u})$ is sufficiently small. If $\operatorname{WTV}(a(\bar{\lambda}))=0$, then $a(\bar{\lambda})$ is constant and we have no constraints; this is the famous Nishida existence theorem, [18].

The plan of the paper is the following. The next Section 2 contains the main result. Section 3 introduces the wave curves; here, differently from [2], we exploit the Riemann coordinates as in [19]. Some technical result about the total variation referred to these coordinates is provided in the Appendix. Section 4 is concerned with the Riemann problem and the next Section 5 with interactions of waves. The treatment of the matter in both sections differs from that in [2] our new proofs are mostly given by geometric considerations, following again [19]. The wave-front algorithm is defined in Section 6. apart from the introduction of many linear functionals and interaction potentials, it is analogous to that contained in [7. Sections 7 and 8 aim at proving that the functional $F$ introduced in Section 6 is decreasing for a suitable choice of the parameters occurring in its definition. Such parameters are chosen explicitly in (8.16), which will be helpful in understanding the argument in [15, [16].

The core of this paper is the lengthy Section 9. There, we explain in detail the method of decomposition by paths and provide the most important properties of paths; notice 
that the generation order defined there differs from that of [7], [2]. The decomposition by path is used in the following Section 10 to deduce further estimates along paths. In the last technical Section 11 we prove at last the convergence of the approximate solutions.

2. The main result. We consider the system of conservation laws (1.1) for $t>0$ and $x \in \mathbb{R}$. The mass density fraction $\lambda$ of vapor in the fluid is valued in $[0,1]$, with $\lambda=0$ characterizing the liquid and $\lambda=1$ the vapor phase; intermediate values of $\lambda$ model mixtures of the two pure phases. We define

$$
p(v, \lambda)=\frac{a^{2}(\lambda)}{v}
$$

for a $\mathbf{C}^{\mathbf{1}}$ function $a$ defined on $[0,1]$ and satisfying for every $\lambda \in[0,1]$,

$$
a(\lambda)>0, \quad a^{\prime}(\lambda)>0 .
$$

In the case of a two-mode reaction, an explicit example of the function $a$ is given in [14]. By (2.1), (2.2) the system (1.1) is strictly hyperbolic for $(v, u, \lambda) \in \Omega=(0,+\infty) \times \mathbb{R} \times[0,1]$ with characteristic speeds $e_{1}=-a(\lambda) / v, e_{2}=0, e_{3}=a(\lambda) / v$; the characteristic speeds $e_{1}, e_{3}$ are genuinely nonlinear while $e_{2}$ is linearly degenerate. We refer to [7, 9], 21] for definitions and general information on conservation laws. Because of (2.2) we can take as variables either $(v, u, \lambda)$ or $(p, u, \lambda)$.

We denote by $\operatorname{TV}(f)$ the total variation of a scalar function $f: \mathbb{R} \rightarrow \mathbb{R}$. If $f$ is strictly positive we also use the weighted total variation of $f$, which is motivated by the definition of the strength for the waves of the second family, 2]:

$$
\operatorname{WTV}(f)=2 \sup \sum_{j=1}^{n} \frac{\left|f\left(x_{j}\right)-f\left(x_{j-1}\right)\right|}{f\left(x_{j}\right)+f\left(x_{j-1}\right)} .
$$

Here the supremum is taken over all $n \geq 1$ and $(n+1)$-tuples of points $x_{j}, x_{o}<x_{1}<$ $\ldots<x_{n}$. The relationships between $\operatorname{WTV}(f), \operatorname{TV}(f)$ and $\operatorname{TV}(\log (f))$ are the following, [2]:

$$
\frac{\inf f}{\sup f} \mathrm{TV}(\log (f)) \leq \frac{1}{\sup f} \mathrm{TV}(f) \leq \mathrm{WTV}(f) \leq \mathrm{TV}(\log (f)) \leq \frac{1}{\inf f} \mathrm{TV}(f) .
$$

If in addition $f$ is continuous, then $\operatorname{WTV}(f)=\mathrm{TV}(\log (f))$. For waves of the first and third family we use the Riemann coordinates $r, s$ associated to the liquid phase; in particular, in the plane $v u$ we use the induced distance (3.3). As a consequence, we denote by $\operatorname{TV}(v, u)$ the total variation of $(v, u)$ computed by using such a distance.

Now, we can state our main result. We denote the initial data for system (1.1) by

$$
(\bar{v}(x), \bar{u}(x), \bar{\lambda}(x))
$$

and assume that

$$
\bar{v}(x) \geq v_{0}>0 \quad \text { and } \quad 0 \leq \bar{\lambda}(x) \leq 1,
$$

for some constant $v_{0}$ and every $x \in \mathbb{R}$. Moreover, define $\bar{a}(x)=a(\lambda(x))$ and

$$
\bar{a}_{*}=\inf _{x \in \mathbb{R}} \bar{a}(x), \quad \bar{a}^{*}=\sup _{x \in \mathbb{R}} \bar{a}(x), \quad[\bar{a}]_{*}=\frac{\bar{a}^{*}-\bar{a}_{*}}{\bar{a}^{*}+\bar{a}_{*}}, \quad\langle\bar{a}\rangle \doteq \frac{1}{2} \frac{\mathrm{WTV}(\bar{a})}{1-[\bar{a}]_{*}} .
$$

The main result in this paper is the following theorem. 
Theorem 2.1. Consider the system (1.1) under the assumptions (2.1)-(2.2) and initial data (2.3) satisfying (2.4). There exists a positive constant $c \in(0,1)$ such that if

$$
\langle\bar{a}\rangle \mathrm{e}^{\langle\bar{a}\rangle} \cdot \frac{2-c}{(1-c)^{2}}<\frac{1}{2}
$$

holds and

$$
\operatorname{WTV}(\bar{a}) \cdot \operatorname{TV}(\bar{v}, \bar{u})
$$

is sufficiently small, then the initial-value problem (1.1), (2.3) has a weak entropy solution $(v, u, \lambda)$ which is defined for all $t>0$. Moreover, $v$ is bounded away from zero and the variation of $(v(t, \cdot), u(t, \cdot))$ is uniformly bounded for every $t>0$.

Now, we make several comments on the previous statement. The constant $c$ comes from (5.23) and depends on the slopes of the shock curves; clearly, such slopes also depend on $\lambda$. More precisely, once the strengths of all interacting shock waves in the approximated solution are proved to be less than a constant $M$, then we find $c$ as a function of $M, \bar{a}_{*}$ and $\bar{a}^{*}$ so that (5.23) holds for all such waves. In Section 11 we shall see that $M$ can be chosen as

$$
M=2\left|\left(\bar{v}_{+\infty}, \bar{u}_{+\infty}\right)-\left(\bar{v}_{-\infty}, \bar{u}_{-\infty}\right)\right|+\frac{24}{1-[\bar{a}]_{*}} \mathrm{TV}(\bar{v}, \bar{u})
$$

which only depends on the initial data. An estimate from above for $c$ is then provided in (5.24). It is worth noticing that the existence of $c$ is granted even if arbitrary large initial data $(\bar{v}, \bar{u})$ are given (cf. [2], [13]).

The condition (2.6) regards the size of 2-waves. If $\bar{\lambda}$ is constant, then $c$ solely depends on $M$ and (2.6) is trivially satisfied; in this way we recover the famous result by Nishida and Smoller [19].

At last, a precise threshold for the smallness of the quantity $\operatorname{WTV}(\bar{a}) \cdot \operatorname{TV}(\bar{v}, \bar{u})$ is provided in Remark 11.9.

3. Wave curves. In this section we fix two constants $0<a_{*}<a^{*}$ and consider constant states $\lambda$ such that

$$
a_{*} \leq a(\lambda) \leq a^{*} .
$$

In particular one may choose $a_{*}=a(0)$ and $a^{*}=a(1)$; we denote $[a]_{*}=\frac{a^{*}-a_{*}}{a^{*}+a_{*}}$. The Riemann coordinates $r$ and $s$ for the first two equations in (1.1) with respect to $a_{*}$ are [19]

$$
r=u-a_{*} \log p, \quad s=u+a_{*} \log p .
$$

In the plane $v u$ we introduce the distance

$$
\left|\left(v^{\prime}, u^{\prime}\right)-(v, u)\right|=\max \left\{\left|r\left(v^{\prime}, u^{\prime}\right)-r(v, u)\right|,\left|s\left(v^{\prime}, u^{\prime}\right)-s(v, u)\right|\right\} .
$$

The total variation of a function $(v, u): \mathbb{R} \rightarrow \mathbb{R}^{2}$ is then defined by using the distance (3.3).

For families 1 and 3 the shock-rarefaction curves for (1.1) can be parameterized by $v$, 1]; here we use coordinates $(p, u, \lambda)$. Denote $a_{o}=a\left(\lambda_{o}\right)$; the wave curves through 
$\left(p_{o}, u_{o}, \lambda_{o}\right) \in \Omega$ are

$$
\begin{aligned}
& 1: u-u_{o}=\left\{\begin{array}{lll}
a_{o}\left(\sqrt{\frac{p_{o}}{p}}-\sqrt{\frac{p}{p_{o}}}\right) & p>p_{o} & \text { shock } \\
a_{o} \log \frac{p_{o}}{p} & p<p_{o} & \text { rarefaction }
\end{array} \quad \text { and } \lambda=\lambda_{o},\right. \\
& 2: \quad p=p_{o} \quad \text { and } u=u_{o} \text {, }
\end{aligned}
$$

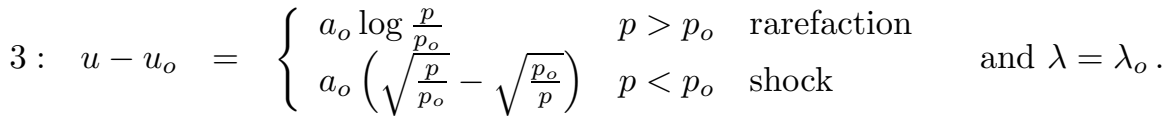

In the phase space $(p, u, \lambda)$ the 2 -wave curves are then orthogonal to 1- and 3-wave curves.

Lemma 3.1. Consider a constant state $U_{o}=\left(p_{o}, u_{o}, \lambda_{o}\right) \in \Omega$. There exists a function $f_{o}$ defined in $(-\infty, 0]$ and depending on $\lambda_{o}$ such that the wave curves for (1.1) through $U_{o}$ are

$$
\begin{aligned}
& 1: s-s_{o}=\left\{\begin{array}{lll}
f_{o}\left(r-r_{o}\right) & r<r_{o} & \text { shock } \\
\frac{a_{o}-a_{*}}{a_{o}+a_{*}}\left(r-r_{o}\right) & r>r_{o} & \text { rarefaction }
\end{array} \quad \text { and } \lambda=\lambda_{o},\right. \\
& \text { 2: } \quad r=r_{o} \quad \text { and } s=s_{o} \text {, } \\
& 3: r-r_{o}=\left\{\begin{array}{lll}
\frac{a_{o}-a_{*}}{a_{o}+a_{*}}\left(s-s_{o}\right) & s>s_{o} & \text { rarefaction } \\
f_{o}\left(s-s_{o}\right) & s<s_{o} & \text { shock }
\end{array} \quad \text { and } \lambda=\lambda_{o} .\right.
\end{aligned}
$$

The function $f_{o}$ is smooth and satisfies $f_{o}^{\prime}(0)=\frac{a_{o}-a_{*}}{a_{o}+a_{*}}, f_{o}^{\prime}(x) \sim 1$ for $x \rightarrow-\infty$ and

$$
\frac{a_{o}-a_{*}}{a_{o}+a_{*}} \leq f_{o}^{\prime}(x)<1
$$

Moreover $f_{o}^{\prime \prime}(0)=0, f_{o}$ is concave and

$$
\left|f_{o}^{\prime \prime}(x)\right| \leq \frac{a_{*}}{\left(a_{o}+a_{*}\right)^{2}} .
$$

Proof. The parametrization for the rarefaction curves follows directly from (3.4). In the case of 1-rarefactions the conditions $p_{o} / p>1$ and then $u-u_{o}>0$ imply $r-r_{o}>0$. The case of 3-rarefactions is analogous.

Consider then the case of 1 -shock curves. We define $y=\frac{1}{2} \log \frac{p_{o}}{p}$ so that from (3.4) we have $y<0$ on the shock curve and

$$
\begin{aligned}
r-r_{o} & =2 a_{*} y+2 a_{o} \sinh y \doteq g(y), \\
s-s_{o} & =-2 a_{*} y+2 a_{o} \sinh y .
\end{aligned}
$$

We have $g^{\prime}(y) \geq 2\left(a_{o}+a_{*}\right)>0$ for any $y$; then the function $g$ is invertible and (3.7) reads $y=g^{-1}\left(r-r_{o}\right)$. Inserting this expression in (3.8) gives

$$
s-s_{o}=-2 a_{*} g^{-1}\left(r-r_{o}\right)+2 a_{o} \sinh \left(g^{-1}\left(r-r_{o}\right)\right) \doteq f_{o}\left(r-r_{o}\right) .
$$

Arguing as for 1-rarefactions we see that this must hold for $r<r_{o}$. The case of 3-shock curves is analogous: one defines $z=\frac{1}{2} \log \frac{p}{p_{o}}$ and finds $r-r_{o}=-2 a_{*} z+2 a_{o} \sinh z$, $s-s_{o}=2 a_{*} z+2 a_{o} \sinh z$. 


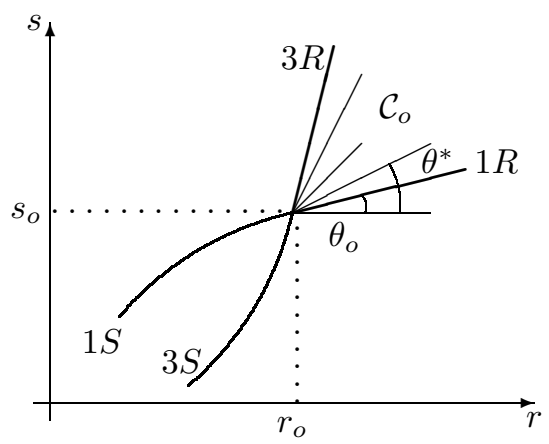

FIG. 1. Lax shock-rarefaction curves.

Concerning the properties of the function $f_{o}(x)=2 a_{o} \sinh \left(g^{-1}(x)\right)-2 a_{*} g^{-1}(x)$, we remark that from the inverse function theorem we have, for $y=g^{-1}(x)$,

$$
f_{o}^{\prime}(x)=\frac{a_{o} \cosh (y)-a_{*}}{a_{o} \cosh (y)+a_{*}}
$$

whence the asymptotic behavior of $f_{o}^{\prime}$. Moreover

$$
f_{o}^{\prime \prime}(x)=\frac{a_{o} a_{*} \sinh y}{\left(a_{o} \cosh y+a_{*}\right)^{3}},
$$

whence the concavity of $f_{o}$. At last (3.6) follows from

$$
\left|f_{o}^{\prime \prime}(x)\right| \leq \frac{a_{*}|\tanh y|}{\left(a_{o} \cosh y+a_{*}\right)^{2}} \leq \frac{a_{*}}{\left(a_{o}+a_{*}\right)^{2}} .
$$

REMARK 3.2. If we extend the function $f_{o}$ by $f_{o}(x)=\frac{a_{o}-a_{*}}{a_{o}+a_{*}} x$ for $x \geq 0$, then $f_{o}$ : $\mathbb{R} \rightarrow \mathbb{R}$ is a $\mathbf{C}^{2}$ function and the shock-rarefaction curves in the plane $(r, s)$ are written as

$$
\begin{aligned}
& 1: s-s_{o}=f_{o}\left(r-r_{o}\right), \quad \begin{cases}r<r_{o} & \text { shock } \\
r>r_{o} & \text { rarefaction, }\end{cases} \\
& 3: r-r_{o}=f_{o}\left(s-s_{o}\right), \quad \begin{cases}s<s_{o} & \text { shock } \\
s>s_{o} & \text { rarefaction. }\end{cases}
\end{aligned}
$$

Rarefaction curves of the families 1 and 3 are orthogonal in the plane $(r, s)$ if $a_{o}=a_{*}$; the same happens for the $p$-system in the isothermal case, [18. In general, since $0 \leq$ $\frac{a_{o}-a_{*}}{a_{o}+a_{*}} \leq \frac{a^{*}-a_{*}}{a^{*}+a_{*}}<1$, the 1-rarefaction (3-rarefaction) curves form with the $r$ (respectively $s)$ axis an angle $\theta_{o} \in\left[0, \theta^{*}\right]$ with $\theta^{*}<\pi / 4$, where

$$
\tan \left(\theta_{o}\right)=\frac{a_{o}-a_{*}}{a_{o}+a_{*}} \doteq k_{o}, \quad \tan \left(\theta^{*}\right)=\frac{a^{*}-a_{*}}{a^{*}+a_{*}}=[a]_{*} ;
$$

see Figure 1. Observe that $\theta_{o}$ increases with $\lambda_{o}$. Moreover, the sector $\mathcal{C}_{o}$ centered in $\left(r_{o}, s_{o}\right)$ and bounded by the 1 - and 3-rarefaction curves with $\lambda=1$ contains no rarefaction curves. Also observe that while every rarefaction curve is obtained by a rarefaction curve of the isothermal $p$-system by a rotation ( of $\pm \theta_{o}$ ), that is not the case for shock curves.

We now define the wave strengths; for 1 - and 3 -waves we use Riemann invariants. 
Definition 3.3. The strength of an $i$-wave joining $U_{o}=\left(p_{o}, u_{o}, \lambda_{o}\right)$ on the left to $U=(p, u, \lambda)$ on the right is defined for $i=1,2,3$ by

$$
\varepsilon_{1}=r-r_{o}, \quad \varepsilon_{2}=2 \frac{a-a_{o}}{a+a_{o}}, \quad \varepsilon_{3}=s-s_{o} .
$$

Observe that $\left|\varepsilon_{2}\right| \leq 2[a]_{*}<2$; rarefactions have positive strengths while shocks have negative strengths. In [1] for 1- and 3-waves the following strengths were used:

$$
\tilde{\varepsilon}_{1}=\frac{1}{2} \log \left(\frac{v}{v_{o}}\right), \quad \tilde{\varepsilon}_{3}=\frac{1}{2} \log \left(\frac{v_{o}}{v}\right) .
$$

We have $\tilde{\varepsilon}_{1}=\frac{1}{2} \log \left(\frac{p_{o}}{p}\right), \tilde{\varepsilon}_{3}=\frac{1}{2} \log \left(\frac{p}{p_{o}}\right)$ so that (3.11) reads $\tilde{\varepsilon}_{1}=q-q_{o}, \tilde{\varepsilon}_{3}=q_{o}-q$ for $q=\frac{1}{2} \log v$. If we define $h(\tilde{\varepsilon})=\tilde{\varepsilon}$ if $\tilde{\varepsilon} \geq 0$ and $h(\tilde{\varepsilon})=\sinh \tilde{\varepsilon}$ if $\tilde{\varepsilon}<0$, then the $i$-wave curve through $U_{o}$ is

$$
u-u_{o}=2 a_{o} h\left(\tilde{\varepsilon}_{i}\right), \quad i=1,3 .
$$

By (3.9) and the definition of strengths, the 1- and 3-wave curves (3.5) are written simply as

$$
1: s-s_{o}=\left\{\begin{array}{ll}
f_{o}\left(\varepsilon_{1}\right) & \varepsilon_{1}<0 \\
k_{o} \varepsilon_{1} & \varepsilon_{1}>0,
\end{array} \quad 3: r-r_{o}= \begin{cases}k_{o} \varepsilon_{3} & \varepsilon_{3}>0 \\
f_{o}\left(\varepsilon_{3}\right) & \varepsilon_{3}<0 .\end{cases}\right.
$$

Lemma 3.4. For $i=1,3$ we have $\varepsilon_{i}=u-u_{o}+2 a_{*} \tilde{\varepsilon}_{i}$. More precisely

$$
\varepsilon_{i}= \begin{cases}2\left(a_{o}+a_{*}\right) \tilde{\varepsilon}_{i}, & \text { if } \varepsilon_{i} \geq 0 \\ 2\left(a_{o} \sinh \left(\tilde{\varepsilon}_{i}\right)+a_{*} \tilde{\varepsilon}_{i}\right), & \text { if } \varepsilon_{i} \leq 0\end{cases}
$$

In the case of shocks the following estimate holds:

$$
\left|\tilde{\varepsilon}_{i}\right| \leq \frac{1}{2\left(a_{o}+a_{*}\right)}\left|\varepsilon_{i}\right| .
$$

Proof. Formula $\varepsilon_{i}=u-u_{o}+2 a_{*} \tilde{\varepsilon}_{i}$ follows directly from (3.10) and (3.11). Formula (3.13) comes from (3.12). The function $y \mapsto f(y)=2\left(a_{o} \sinh (y)+a_{*} y\right)$ for $y \geq 0$ is increasing and $f(y) \geq 2\left(a_{o}+a_{*}\right) y$; therefore its inverse function $x \rightarrow f^{-1}(x)$ satisfies $f^{-1}(x) \leq \frac{1}{2\left(a_{o}+a_{*}\right)} x$ for $x \geq 0$. Then (3.14) follows from (3.13).

4. The Riemann problem. The Riemann problem in conservative variables was studied in [1] here we consider it in Riemann coordinates. We still assume (3.1). Denote the piecewise constant initial data

$$
(p, u, \lambda)(0, x)= \begin{cases}\left(p_{L}, u_{L}, \lambda_{L}\right)=U_{L} & \text { if } x<0 \\ \left(p_{R}, u_{R}, \lambda_{R}\right)=U_{R} & \text { if } x>0\end{cases}
$$

for states $U_{L}$ and $U_{R}$ in $\Omega$. As in (3.9) denote moreover

$$
k_{L}=\frac{a_{L}-a_{*}}{a_{L}+a_{*}}=\tan \left(\theta_{L}\right), \quad k_{R}=\frac{a_{R}-a_{*}}{a_{R}+a_{*}}=\tan \left(\theta_{R}\right) .
$$

Notice that

$$
\frac{k_{R}-k_{L}}{1-k_{R} k_{L}}=\frac{a_{R}-a_{L}}{a_{R}+a_{L}}=\frac{\sin \left(\theta_{R}-\theta_{L}\right)}{\cos \left(\theta_{R}+\theta_{L}\right)} .
$$




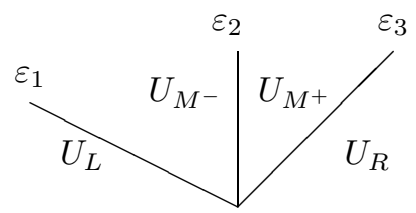

Fig. 2. The Riemann problem.

Proposition 4.1. For any $U_{L}, U_{R}$ in $\Omega$ the Riemann problem (1.1), (4.1) has a unique $\Omega$-valued solution consisting of Lax waves. If $\varepsilon_{i}, i=1,2,3$, are the strengths of the waves, then

$$
\left|\varepsilon_{1}\right|+\left|\varepsilon_{3}\right| \leq \frac{2}{1-\max \left\{k_{L}, k_{R}\right\}}\left(\left|r_{R}-r_{L}\right|+\left|s_{R}-s_{L}\right|\right) .
$$

Proof. The existence of a unique solution is proved easily as in [18, [19]. We now prove (4.4); see Figure 2, Denote by $U_{M^{-}}, U_{M^{+}}$the states in the solution of the Riemann problem between $U_{L}$ and the contact discontinuity (resp., between the contact discontinuity and $U_{R}$ ). Then $u_{M^{-}}=u_{M^{+}} \doteq u_{M}$ and $p_{M^{-}}=p_{M^{+}} \doteq p_{M}$.

If $\varepsilon_{1} \cdot \varepsilon_{3}>0$, then the curve composed by the two wave curves has both $r$ and $s$ either increasing or decreasing; therefore

$$
\left|\varepsilon_{1}\right|+\left|\varepsilon_{3}\right|=\left|r_{M}-r_{L}\right|+\left|s_{R}-s_{M}\right| \leq\left|r_{R}-r_{L}\right|+\left|s_{R}-s_{L}\right| .
$$

Consider the case $\varepsilon_{1}>0, \varepsilon_{3}<0$; see Figure 3(a). Let $V$ be the point in the plane $(r, s)$ where the rarefaction curve through $L M$ and the line $r-r_{R}=s-s_{R}$ meet. Then $\left|\varepsilon_{1}\right|=r_{M}-r_{L}<r_{V}-r_{L}$ and $\left|\varepsilon_{3}\right|=s_{M}-s_{R}<s_{V}-s_{R}$. We compute

$$
\begin{aligned}
r_{V}-r_{L} & =\frac{1}{1-k_{L}}\left(r_{R}-r_{L}-\left(s_{R}-s_{L}\right)\right), \\
s_{V}-s_{R} & =\frac{1}{1-k_{L}}\left(k_{L}\left(r_{R}-r_{L}\right)-\left(s_{R}-s_{L}\right)\right)
\end{aligned}
$$

and so

$$
\begin{aligned}
\left|\varepsilon_{1}\right|+\left|\varepsilon_{3}\right| & \leq \frac{1}{1-k_{L}}\left(\left(1+k_{L}\right)\left|r_{R}-r_{L}\right|+2\left|s_{R}-s_{L}\right|\right) \\
& \leq \frac{2}{1-k_{L}}\left(\left|r_{R}-r_{L}\right|+\left|s_{R}-s_{L}\right|\right) .
\end{aligned}
$$

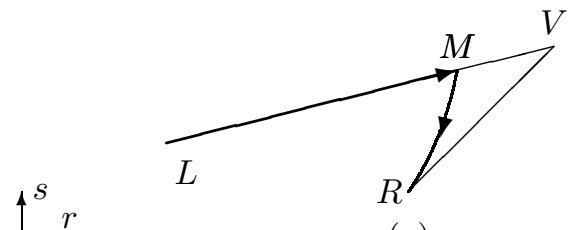

(a)

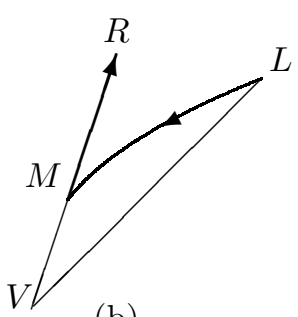

(b)

FIG. 3. Riemann problem. Cases (a): $1 R-3 S$ and (b): $1 S-3 R$. 
At last consider the case $\varepsilon_{1}<0, \varepsilon_{3}>0$; see Figure 3(b). Let $V$ be the point in the plane $(r, s)$ where the rarefaction curve through $M R$ meets the line $r-r_{L}=s-s_{L}$. Then $\left|\varepsilon_{1}\right|=\left|r_{M}-r_{L}\right|<r_{L}-r_{V}$ and $\left|\varepsilon_{3}\right|=s_{R}-s_{M}<s_{R}-s_{V}$. By the previous case and changing $R$ with $L, r$ with $s$, we find

$$
\begin{aligned}
& r_{V}-r_{L}=\frac{1}{1-k_{R}}\left(r_{R}-r_{L}-k_{R}\left(s_{R}-s_{L}\right)\right), \\
& s_{V}-s_{R}=\frac{1}{1-k_{R}}\left(r_{R}-r_{L}-\left(s_{R}-s_{L}\right)\right)
\end{aligned}
$$

and so (4.4) follows by

$$
\begin{aligned}
\left|\varepsilon_{1}\right|+\left|\varepsilon_{3}\right| & \leq \frac{1}{1-k_{R}}\left(2\left|r_{R}-r_{L}\right|+\left(1+k_{R}\right)\left|s_{R}-s_{L}\right|\right) \\
& \leq \frac{2}{1-k_{R}}\left(\left|r_{R}-r_{L}\right|+\left|s_{R}-s_{L}\right|\right) .
\end{aligned}
$$

Since $k_{L}, k_{R} \leq[a]_{*}$ then (4.4) implies $\left|\varepsilon_{1}\right|+\left|\varepsilon_{3}\right| \leq \frac{2}{1-[a]_{*}}\left(\left|r_{R}-r_{L}\right|+\left|s_{R}-s_{L}\right|\right)$. An estimate involving the conserved variables is obtained from (4.4) by recalling (3.2):

$$
\left|\varepsilon_{1}\right|+\left|\varepsilon_{3}\right| \leq \frac{4}{1-\max \left\{k_{L}, k_{R}\right\}}\left(\left|u_{R}-u_{L}\right|+a_{*}\left|\log p_{R}-\log p_{L}\right|\right) .
$$

In general, assume $p \geq \bar{p}>0$; if $J$ is the Jacobian matrix of the transformation $(p, u) \rightarrow$ $(r, s)$, then $\operatorname{det} J=2 a_{*} / p$. It follows that if $0<\underline{p}<\bar{p}$ are fixed numbers, there exists a constant $C_{1}>0$ depending on $\underline{p}, \bar{p}$ and on the function $a$ such that if $\left(p_{L}, u_{L}\right),\left(p_{R}, u_{R}\right) \in$ $\Omega$, with $\underline{p}<p_{L}<\bar{p}$ and $\underline{p}<p_{R}<\bar{p}$, then $\left|\varepsilon_{1}\right|+\left|\varepsilon_{2}\right|+\left|\varepsilon_{3}\right| \leq C_{1}\left|U_{L}-U_{R}\right|$.

5. Interactions. In this section we consider the interactions of pairs of waves under assumption (3.1). First, we study the case when a 2-wave is involved, and then the interactions of 1 - and 3 -waves.

5.1. Interactions with a 2-wave. The following result was proved in [1] using Lax waves for the conservative variables; in Riemann coordinates it can be obtained easily by geometric arguments. We leave the proof to the reader.

Lemma 5.1. Denote by $\lambda_{L}, \lambda_{R}$ the side states of a 2-wave. The interactions of 1- or 3 -waves with a 2 -wave give rise to the following four patterns of solutions:

(i) $C_{2} R_{1}, a_{L}<a_{R}$ and $R_{3} C_{2}, a_{L}>a_{R} \rightarrow R_{1} C_{2} R_{3}$;

(ii) $C_{2} R_{1}, a_{L}>a_{R} \rightarrow R_{1} C_{2} S_{3}$ and $R_{3} C_{2}, a_{L}<a_{R} \rightarrow S_{1} C_{2} R_{3}$;

(iii) $C_{2} S_{1}, a_{L}<a_{R}$ and $S_{3} C_{2}, a_{L}>a_{R} \rightarrow S_{1} C_{2} S_{3}$;

(iv) $C_{2} S_{1}, a_{L}>a_{R} \rightarrow S_{1} C_{2} R_{3}$ and $S_{3} C_{2}, a_{L}<a_{R} \rightarrow R_{1} C_{2} S_{3}$.

In the next proposition we estimate the strengths of the waves arising in an interaction between a 2-wave $\delta_{2}$ and a wave $\delta_{i}$. The contact discontinuity has a damping effect on the reflected wave $\varepsilon_{j}$, namely $\left|\varepsilon_{j}\right| \leq \frac{1}{2}\left|\delta_{2} \delta_{i}\right|$, except in the case (ii), a rarefaction entering in a more gaseous phase, where the magnification effect $\left|\varepsilon_{j}\right| \geq \frac{1}{2}\left|\delta_{2} \delta_{i}\right|$ takes place. 
Proposition 5.2. Assume that a 1 - or a 3 -wave of strength $\delta_{i}$ interacts with a 2 -wave of strength $\delta_{2}$. Then, the strengths $\varepsilon_{i}$ of the transmitted wave, $\varepsilon_{j}$ of the reflected wave and $\varepsilon_{2}$ of the outgoing contact discontinuity satisfy

$$
\begin{aligned}
\varepsilon_{2} & =\delta_{2}, \\
\left|\varepsilon_{i}-\delta_{i}\right| & \leq\left|\varepsilon_{j}\right| .
\end{aligned}
$$

The quadratic interaction estimates are of two types:

$C D 1$. In all cases but (ii), i.e., $C_{2} R_{1}, a_{L}>a_{R}$ and $R_{3} C_{2}, a_{L}<a_{R}$, we have

$$
\begin{aligned}
\left|\varepsilon_{j}\right| & \leq \frac{1}{2}\left|\delta_{2} \delta_{i}\right|, \quad i, j=1,3, i \neq j, \\
\left|\varepsilon_{1}\right|+\left|\varepsilon_{3}\right| & \leq\left|\delta_{i}\right|+\left|\delta_{2} \delta_{i}\right| .
\end{aligned}
$$

CD2. In case (ii), i.e., $C_{2} R_{1}, a_{L}>a_{R}$ and $R_{3} C_{2}, a_{L}<a_{R}$, we have

$$
\begin{aligned}
\left|\varepsilon_{j}\right| & \leq \frac{1}{2} \frac{1}{1-[a]_{*}}\left|\delta_{2} \delta_{i}\right|, \quad i, j=1,3, i \neq j, \\
\left|\varepsilon_{j}\right| & \geq \frac{1}{2}\left|\delta_{2} \delta_{i}\right| \\
\left|\varepsilon_{1}\right|+\left|\varepsilon_{3}\right| & \leq\left|\delta_{i}\right|+\frac{1}{1-[a]_{*}}\left|\delta_{2} \delta_{i}\right| .
\end{aligned}
$$

Proof. We consider the case $i=1$; the case $i=3$ is analogous. First observe that (5.1) is a consequence of Definition 3.3 the estimate (5.2) shall follow by simple geometric consideration, while (5.3) shall be a consequence of a similar result in [1], namely

$$
\left|\tilde{\varepsilon}_{3}\right| \leq \frac{1}{2}\left|\delta_{2} \tilde{\delta}_{1}\right|
$$

According to Lemma 5.1 we distinguish four cases. The notation is as in Figure 4, the interaction diagrams as in Figure 5. For brevity we write here $U$ for the projection of $U$ on the plane $(r, s)$; the length of a segment joining two points $A$ and $B$ in the $(r, s)$ plane is denoted simply by $A B$.

(i) $C_{2} R_{1}, a_{L}<a_{R}$. We have $\delta_{1}>0, \varepsilon_{1}>0, \varepsilon_{3}>0$ : all waves are rarefactions. Moreover $\delta_{1}=U_{M} C, \varepsilon_{1}=U_{M} D, \varepsilon_{3}=U_{R} B$; the angle $C U_{L} U_{-}$is $\theta_{L}$, the angle $C U_{M} U_{R}$ is $\theta_{R}$, the angle $B U_{+} U_{R}$ is $\pi / 2-\theta_{R}$. Since both $\left|\varepsilon_{3}\right|=C D \tan \left(\pi / 2-\theta_{R}\right)$

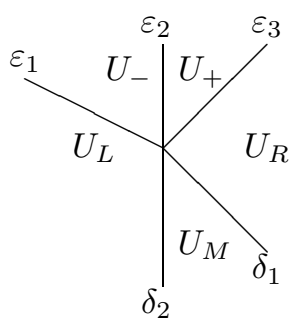

FIG. 4. Interactions. 


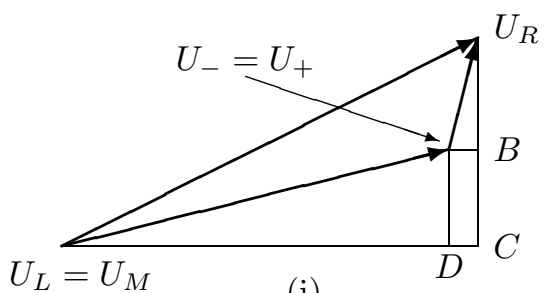

(i)

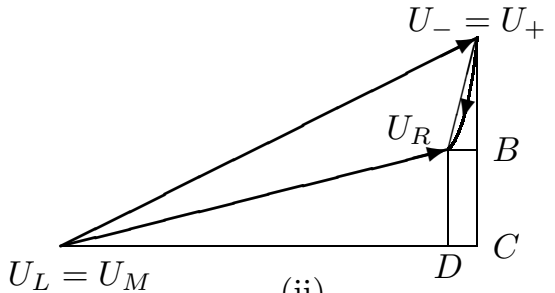

(ii)

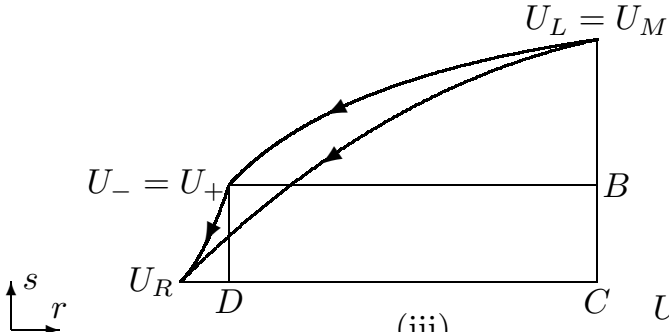

(iii)

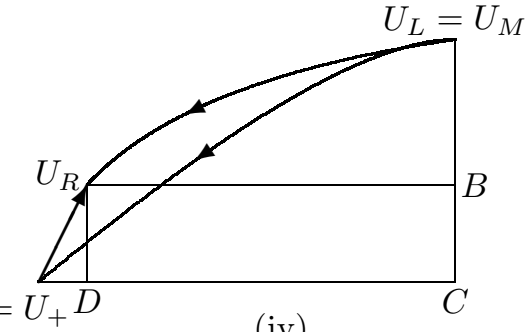

(iv)

FIG. 5. (i): $\delta_{1}>0, a_{L}<a_{R}$; (ii): $\delta_{1}>0, a_{L}>a_{R}$; (iii): $\delta_{1}<0$, $a_{L}<a_{R} ;$ (iv): $\delta_{1}<0, a_{L}>a_{R}$.

and $\left|\varepsilon_{3}\right|=U_{R} C-B C$, using notation as in (4.2) we find

$$
\begin{aligned}
\varepsilon_{3} & =\tan \left(\frac{\pi}{2}-\theta_{R}\right) \cdot\left(\delta_{1}-\varepsilon_{1}\right)=\frac{1}{k_{R}}\left(\delta_{1}-\varepsilon_{1}\right), \\
\varepsilon_{3} & =\tan \left(\theta_{R}\right) \delta_{1}-\tan \left(\theta_{L}\right) \varepsilon_{1}=k_{R} \delta_{1}-k_{L} \varepsilon_{1} .
\end{aligned}
$$

From (5.9) we find $\delta_{1}-\varepsilon_{1}=k_{R} \varepsilon_{3}$, which proves (5.2). Again from (5.9) we deduce $\varepsilon_{1}=\delta_{1}-k_{R} \varepsilon_{3}$; we plug that into the second formula above and find by (4.3),

$$
\varepsilon_{3}=\frac{k_{R}-k_{L}}{1-k_{R} k_{L}} \delta_{1}=\frac{a_{R}-a_{L}}{a_{R}+a_{L}} \delta_{1}=\frac{1}{2} \delta_{2} \delta_{1},
$$

which proves (5.3). Moreover $\varepsilon_{1}=\delta_{1}-\frac{k_{R}}{2} \delta_{2} \delta_{1}$ and so (5.4) follows from

$$
\varepsilon_{1}+\varepsilon_{3}=\delta_{1}+\frac{1-k_{R}}{2} \delta_{2} \delta_{1}
$$

(ii) $C_{2} R_{1}, a_{L}>a_{R}$. We have $\delta_{1}>0, \varepsilon_{1}>0, \varepsilon_{3}<0$. Moreover $\delta_{1}=U_{M} D, \varepsilon_{1}=U_{M} C$, $\varepsilon_{3}=U_{+} B$. The angle $C U_{M} U_{R}$ is $\theta_{R}$, the angle $C U_{L} U_{-}$is $\theta_{L}$, and the angle $B U_{R} U_{+}$ is $\alpha \geq \frac{\pi}{4}$ because $f_{o}^{\prime}(y)$ is asymptotic to $y$; see Lemma 3.1. Consider the triangle $B U_{R} U_{+}$. Since the angle $B U_{R} U_{+}$is larger than $\pi / 4$, then $\left|\varepsilon_{1}-\delta_{1}\right| \leq\left|\varepsilon_{3}\right|$, whence (5.2).

The shock curve starts from the point $U_{+}$forming an angle $\theta_{R}$ with the vertical line. Then $\left|\varepsilon_{3}\right| \leq C D \tan \left(\frac{\pi}{2}-\theta_{R}\right)$ since the shock curve is convex. Moreover $\left|\varepsilon_{3}\right|=U_{+} C-B C$ as above and then

$$
\begin{aligned}
& \left|\varepsilon_{3}\right| \leq \tan \left(\frac{\pi}{2}-\theta_{R}\right) \cdot\left(\varepsilon_{1}-\delta_{1}\right)=\frac{1}{k_{R}}\left(\varepsilon_{1}-\delta_{1}\right), \\
& \left|\varepsilon_{3}\right|=\tan \left(\theta_{L}\right) \varepsilon_{1}-\tan \left(\theta_{R}\right) \delta_{1}=k_{L} \varepsilon_{1}-k_{R} \delta_{1} .
\end{aligned}
$$


Then $k_{R}\left|\varepsilon_{3}\right| \leq\left|\varepsilon_{1}-\delta_{1}\right|=\varepsilon_{1}-\delta_{1}$,

$$
\varepsilon_{1}=\frac{1}{k_{L}}\left(\left|\varepsilon_{3}\right|+k_{R} \delta_{1}\right)
$$

and so by (4.3),

$$
\left|\varepsilon_{3}\right| \geq \frac{k_{L}-k_{R}}{1-k_{R} k_{L}} \delta_{1}=\frac{1}{2} \delta_{2} \delta_{1}
$$

which proves (5.6). We now put (5.10) into (5.2), which was just proved to get

$$
\left|\varepsilon_{3}\right| \leq \frac{k_{L}-k_{R}}{1-k_{L}} \delta_{1}=\frac{1}{2} \cdot \frac{a_{L}+a_{R}}{a_{R}+a_{*}}\left|\delta_{2} \delta_{1}\right| .
$$

Next, from (5.10) and (5.11) we obtain

$$
\begin{aligned}
\left|\varepsilon_{1}\right|+\left|\varepsilon_{3}\right| & =\frac{1+k_{L}}{k_{L}}\left|\varepsilon_{3}\right|+\frac{k_{R}}{k_{L}}\left|\delta_{1}\right| \\
& \leq \frac{1+k_{L}}{k_{L}} \frac{1}{2} \frac{a_{L}+a_{R}}{a_{R}+a_{*}}\left|\delta_{2} \delta_{1}\right|+\left|\delta_{1}\right|+\left(\frac{k_{R}}{k_{L}}-1\right)\left|\delta_{1}\right| \\
& =\left|\delta_{1}\right|+\left(\frac{1+k_{L}}{2 k_{L}} \frac{a_{L}+a_{R}}{a_{R}+a_{*}}+\frac{k_{R}-k_{L}}{2 k_{L}} \frac{a_{L}+a_{R}}{a_{L}-a_{R}}\right)\left|\delta_{2} \delta_{1}\right| \\
& =\left|\delta_{1}\right|+\frac{a_{L}+a_{R}}{a_{R}+a_{*}}\left|\delta_{2} \delta_{1}\right| .
\end{aligned}
$$

From (5.11) and (5.12) we deduce that in case (ii) we have

$$
\left|\varepsilon_{j}\right| \leq \frac{1}{2} \frac{a_{R}+a_{L}}{\min \left\{a_{L}, a_{R}\right\}+a_{*}}\left|\delta_{2} \delta_{i}\right|, \quad\left|\varepsilon_{1}\right|+\left|\varepsilon_{3}\right| \leq\left|\delta_{i}\right|+\frac{a_{R}+a_{L}}{\min \left\{a_{L}, a_{R}\right\}+a_{*}}\left|\delta_{2} \delta_{i}\right|
$$

In order to have uniform estimates, observe that

$$
\frac{a_{R}+a_{L}}{a_{R}+a_{*}}=1+\frac{a_{L}-a_{*}}{a_{R}+a_{*}} \leq 1+\frac{a^{*}-a_{*}}{2 a_{*}}=\frac{1}{1-[a]_{*}}
$$

and an analogous estimate holds changing $a_{R}$ with $a_{L}$. Then

$$
\frac{a_{R}+a_{L}}{\min \left\{a_{L}, a_{R}\right\}+a_{*}} \leq \frac{1}{1-[a]_{*}}
$$

and from (5.13) we obtain (5.5), (5.7). Notice that $1<\frac{1}{1-[a]_{*}}<\frac{1+[a]_{*}}{1-[a]_{*}}=\frac{a^{*}}{a_{*}}$.

(iii) $C_{2} S_{1}, a_{L}<a_{R}$. We have $\delta_{1}<0, \varepsilon_{1}<0, \varepsilon_{3}<0$ : all waves are shocks. Moreover $\left|\delta_{1}\right|-\left|\varepsilon_{1}\right|=\left|\delta_{1}-\varepsilon_{1}\right|=U_{R} D \geq \frac{\left|\varepsilon_{3}\right|}{\tan \left(\frac{\pi}{2}-\theta_{R}\right)}=k_{R}\left|\varepsilon_{3}\right|$. However, as in the previous case, the angle $U_{+} U_{R} D$ is larger than $\pi / 4$, whence (5.2). To prove (5.3), first obtain the easy estimate

$$
\sinh (c x) \leq c \sinh (x), \quad \text { for } 0 \leq c \leq 1 \text { and } x \geq 0 .
$$

Then from (3.13), (5.8) and (5.15) with $c=\left|\delta_{2}\right| / 2$ we obtain

$$
\begin{aligned}
\left|\varepsilon_{3}\right| & =2\left[a_{R} \sinh \left(\left|\tilde{\varepsilon}_{3}\right|\right)+a_{*}\left|\tilde{\varepsilon}_{3}\right|\right] \\
& \leq 2\left[a_{R} \sinh \left(\left|\delta_{2} \tilde{\delta}_{1}\right| / 2\right)+a_{*}\left|\delta_{2} \tilde{\delta}_{1}\right| / 2\right] \\
& \leq \frac{1}{2}\left|\delta_{2}\right| \cdot 2\left[a_{R} \sinh \left(\left|\tilde{\delta}_{1}\right|\right)+a_{*}\left|\tilde{\delta}_{1}\right|\right]=\frac{1}{2}\left|\delta_{2} \delta_{1}\right| .
\end{aligned}
$$

From the above estimates then (5.4) follows from $\left|\varepsilon_{1}\right|+\left|\varepsilon_{3}\right| \leq\left|\delta_{1}\right|+\frac{1-k_{R}}{2}\left|\delta_{2} \delta_{1}\right|$. 
(iv) $C_{2} S_{1}, a_{L}>a_{R}$. We have $\delta_{1}<0, \varepsilon_{1}<0, \varepsilon_{3}>0$. We have $\left|\varepsilon_{1}\right|-\left|\delta_{1}\right|=\left|\delta_{1}-\varepsilon_{1}\right|=$ $U_{+} D=\frac{\left|\varepsilon_{3}\right|}{\tan \left(\frac{\pi}{2}-\theta_{R}\right)}=k_{R}\left|\varepsilon_{3}\right|$, whence (5.2). The estimate (5.3) follows from (3.13), (3.14) and (5.8):

$$
\left|\varepsilon_{3}\right|=2\left(a_{R}+a_{*}\right)\left|\tilde{\varepsilon}_{3}\right| \leq \frac{1}{2}\left|\delta_{2}\right| \cdot 2\left(a_{R}+a_{*}\right)\left|\tilde{\delta}_{1}\right| \leq \frac{1}{2}\left|\delta_{2}\right|\left|\delta_{1}\right| .
$$

Then (5.4) follows from the above estimates: $\left|\varepsilon_{1}\right|+\left|\varepsilon_{3}\right| \leq\left|\delta_{1}\right|+\frac{1+k_{R}}{2}\left|\delta_{2} \delta_{1}\right|$.

REMARK 5.3. In Proposition 5.2 we proved something more than (5.2). In fact in all cases

$$
\begin{aligned}
&\left|\varepsilon_{i}-\delta_{i}\right|=k_{*_{j}}\left|\varepsilon_{j}\right| \quad \text { if } \varepsilon_{j}>0, \\
& k_{*_{j}}\left|\varepsilon_{j}\right| \leq\left|\varepsilon_{i}-\delta_{i}\right| \leq\left|\varepsilon_{j}\right| \quad \text { if } \varepsilon_{j}<0,
\end{aligned}
$$

where $k_{*_{j}}$ is $k_{R}$ or $k_{L}$ according to $j=3$, resp. $j=1$. Also the estimate $\left|\varepsilon_{j}\right|<$ $\frac{1}{2} \frac{1}{1-[a]_{*}}\left|\delta_{2} \delta_{i}\right|$ holds in all cases.

5.2. Interactions of 1 - and 3-waves. We now consider the interactions of waves of the families 1 and 3, arguing as in [19. Let $\lambda_{o}$ be the constant value of $\lambda$ in the interaction, $k_{o}$ as in (3.9) and

$$
l_{o} \doteq \frac{1}{2\left(a_{o}+a_{*}\right)}
$$

Proposition 5.4. Assume that an $i$-wave $W_{i}$ and a $j$-wave $W_{j}, i, j \in\{1,3\}$ interact at time $t>0$. Let $\delta_{i}, \delta_{j}$ (or $\delta_{i}, \delta_{i}^{\prime}$ if they are of the same type) be their strengths, $\varepsilon_{k}$ be the strengths of the outgoing waves, $k=1,3, U_{L}, U_{R}$ the outer states in the interaction.

There exists a constant $0 \leq c_{L R}<1$, depending on $U_{L}$ and $U_{R}$ and uniformly on $\lambda_{o}$, such that the following estimates hold.

(1) $W_{3} W_{1} \rightarrow W_{1} W_{3}$ : then $\varepsilon_{i}=\delta_{i}$ for $i=1,3$.

(2) $S_{i} R_{i}^{\prime} \rightarrow S_{i} S_{j}$ : then $\zeta_{i} \doteq\left|\delta_{i}\right|-\left|\varepsilon_{i}\right|>0$ and $\left|\varepsilon_{j}\right| \leq c_{L R} \zeta_{i}$.

(3) $S_{i} R_{i}^{\prime} \rightarrow R_{i} S_{j}$ : then $\left|\varepsilon_{j}\right| \leq c_{L R}\left|\delta_{i}\right|$.

(4) $S_{i} S_{i}^{\prime} \rightarrow S_{i} R_{j}$ : then

$$
\begin{aligned}
& \left|\varepsilon_{j}\right| \leq \min \left\{c_{L R}\left|\delta_{i}\right|, c_{L R}\left|\delta_{i}^{\prime}\right|, l_{o}\left|\delta_{i} \delta_{i}^{\prime}\right|\right\} \\
& \left|\varepsilon_{i}\right| \leq\left|\delta_{i}\right|+\left|\delta_{i}^{\prime}\right|+k_{o} l_{o}\left|\delta_{i} \delta_{i}^{\prime}\right| .
\end{aligned}
$$

Proof. Every 3 -wave can interact with a 1-wave; waves of the same family interact if and only if at least one of them is a shock. In this case, it is easily proved that if one of the interacting waves is a rarefaction, then the outcome is a shock or a rarefaction of the same family and a shock of the other family. On the other hand, when two shocks interact a shock of the same family is produced together with a rarefaction of the other family. Therefore the cases listed above cover all possibilities.

Below, $U_{M}\left(U_{Q}\right)$ is the state between the two interacting (outgoing) waves; see Figure 6. Apart from the first case we consider the case $i=1, j=3$.

(1) $W_{3} W_{1} \rightarrow W_{1} W_{3}$. If the incoming waves are of different type, then they cross without changing their strengths, as a consequence of the congruence of the Lax curves.

(2) $S_{i} R_{i}^{\prime} \rightarrow S_{i} S_{j}$. Let $\delta_{1}$ (resp. $\varepsilon_{1}, \varepsilon_{3}$ ) be the strength of the shock between $U_{L}$ and $U_{M}$ (resp. $U_{L}$ and $U_{Q}, U_{Q}$ and $U_{R}$ ); the strength of the rarefaction between $U_{M}$ and $U_{R}$ is $\delta_{1}^{\prime}$. 
A simple computation as in the proof of Proposition 5.2 shows that $k_{o}\left|\varepsilon_{3}\right| \leq$ $\left|\delta_{1}\right|-\left|\delta_{1}^{\prime}\right|-\left|\varepsilon_{1}\right|$; however, this estimate is useless to bound $\left|\varepsilon_{3}\right|$ because $k_{o}$ may vanish. Denote $c_{L R}<1$, the slope of the line passing through $Q$ and $M$; of course $c_{L R} \leq f_{o}^{\prime}\left(\delta_{1}\right)$. Then $Q E<Q D=c_{L R} M D$, i.e., $\left|\varepsilon_{3}\right| \leq c_{L R}\left(\left|\delta_{1}\right|-\left|\varepsilon_{1}\right|\right)$.

(3) $S_{i} R_{i}^{\prime} \rightarrow R_{i} S_{j}$. Let $\delta_{1}$ (resp. $\varepsilon_{3}$ ) be the strength of the shock between $U_{L}$ and $U_{M}$ (resp. $U_{Q}$ and $U_{R}$ ); the strength of the rarefaction between $U_{M}$ and $U_{R}\left(U_{L}\right.$ and $U_{Q}$ ) is $\delta_{1}^{\prime}\left(\varepsilon_{1}\right)$. First, observe that the line passing through $L$ and $Q$ is parallel to that through $M$ and $R$; since the slope of the curve through $L$ and $M$ (through $Q$ and $R$ ) is less (resp., greater) than one, it follows that $L H \leq M G$, i.e., $\left|\varepsilon_{1}\right| \leq\left|\delta_{1}^{\prime}\right|$. Next, denote by $c_{L R}$ the slope of the line through $L$ and $M$; also in this case $c_{L R} \leq f_{o}^{\prime}\left(\delta_{1}\right)$. Then, since $L H \leq M G$, we have $Q E=Q H+H D-E D=k_{o} L H+c_{L R} F M-k_{o} M G \leq$ $c_{L R} F M$, i.e., $\left|\varepsilon_{3}\right| \leq c_{L R}\left|\delta_{1}\right|$.

(4) $S_{i} S_{i}^{\prime} \rightarrow S_{i} R_{j}$. Let $\delta_{1}$ (resp. $\delta_{1}^{\prime}, \varepsilon_{1}$ ) be the strength of the shock between $U_{L}$ and $U_{M}$ (resp. $U_{M}$ and $U_{R}, U_{L}$ and $U_{Q}$ ); the strength of the rarefaction between $U_{Q}$ and $U_{R}$ is $\varepsilon_{3}$; see Figure 6 (4). Moreover $U_{E}$ is the state on the 1-shock curve from $U_{L}$ corresponding to the strength $\delta_{1}+\delta_{1}^{\prime}$. The point $E$ lies then below the intersection point of the line $R D$ with the line from $Q$ with slope 1 . From $s_{M}-s_{L}=f_{o}\left(\delta_{1}\right)$, $s_{R}-s_{M}=f_{o}\left(\delta_{1}^{\prime}\right)$ we find $s_{R}-s_{L}=f_{o}\left(\delta_{1}\right)+f_{o}\left(\delta_{1}^{\prime}\right)$; moreover $s_{E}-s_{L}=f_{o}\left(\delta_{1}+\delta_{1}^{\prime}\right)$. By subtraction,

$$
\begin{aligned}
h \doteq s_{R}-s_{E} & =f_{o}\left(\delta_{1}\right)+f_{o}\left(\delta_{1}^{\prime}\right)-f_{o}\left(\delta_{1}+\delta_{1}^{\prime}\right) \\
& =-\int_{0}^{\delta_{1}^{\prime}}\left\{f_{o}^{\prime}\left(\delta_{1}+\tau\right)-f_{o}^{\prime}(\tau)\right\} d \tau \leq \frac{a_{*}}{\left(a_{o}+a_{*}\right)^{2}}\left|\delta_{1} \delta_{1}^{\prime}\right|
\end{aligned}
$$

by (3.6) and Lemma 2.5 in [7]. We have $D E \doteq l^{\prime}<l \doteq Q D$. Then $l=(h+$ $\left.l^{\prime}\right) \tan \left(\theta_{o}\right)<(h+l) \tan \left(\theta_{o}\right)$, whence $l<\frac{k_{o}}{1-k_{o}} h$. As a consequence, $\left|\varepsilon_{3}\right|=h+l^{\prime}<$ $h+l<\frac{1}{1-k_{o}} h \leq \frac{1}{2\left(a_{o}+a_{*}\right)}\left|\delta_{1} \delta_{1}^{\prime}\right|$ by (5.20), i.e., the last estimate in (5.18).

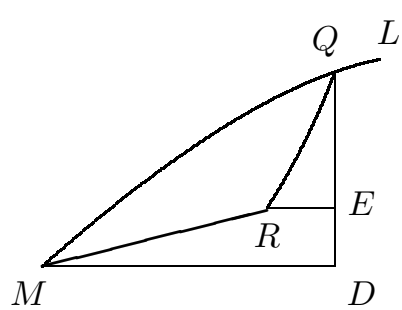

$(2)$
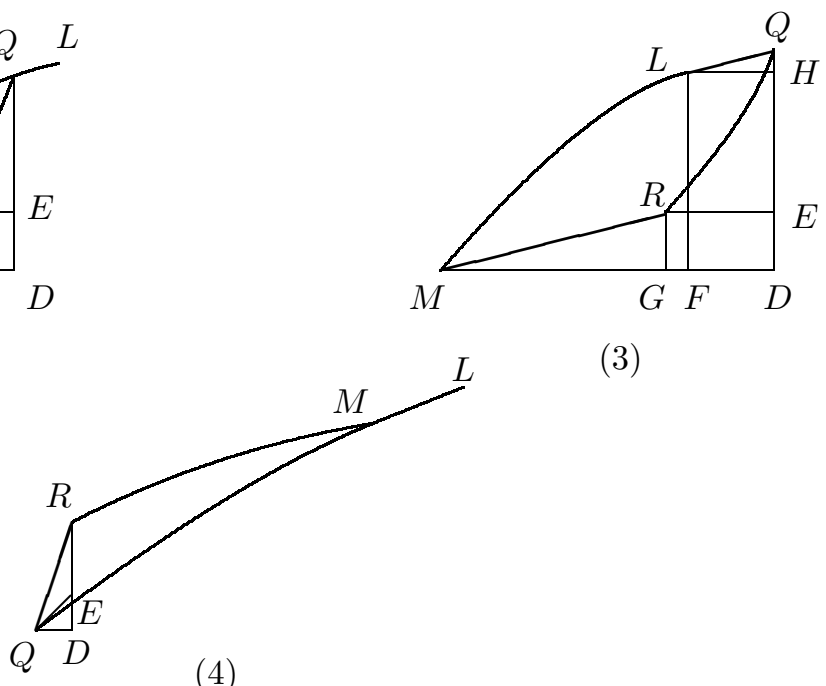

$(3)$

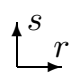

$(4)$

FIG. 6. Interactions. (2): $S_{i} R_{i}^{\prime} \rightarrow S_{i} S_{j}$, (3): $S_{i} R_{i}^{\prime} \rightarrow R_{i} S_{j}$, (4): $S_{i} S_{i}^{\prime} \rightarrow S_{i} R_{j}$ 
Since $\delta_{1}+\delta_{1}^{\prime}=\delta_{1}^{\prime}+\delta_{1}$, we may assume $\left|\delta_{1}^{\prime}\right| \leq\left|\delta_{1}\right|$. Let $E^{\prime}$ be the intersection point of the line $R D$ with the tangent to the shock curve at $Q$ and $\theta$ be the angle $E^{\prime} Q D$. Then we have, for $\delta_{1}^{\prime} \leq \tau \leq 0$,

$$
f_{o}^{\prime}\left(\delta_{1}+\tau\right) \leq f_{o}^{\prime}\left(\varepsilon_{1}\right) \doteq \tan \theta \quad \text { and } \quad f_{o}^{\prime}(\tau) \geq f_{o}^{\prime}(0)=\tan \theta_{o} .
$$

We have $\tan \theta \geq \tan \theta_{o}$ because $f_{o}$ is concave. Hence it follows from (5.20) that

$$
h^{\prime} \doteq s_{R}-s_{E^{\prime}} \leq h \leq\left(\tan \theta-\tan \theta_{o}\right)\left|\delta_{1}^{\prime}\right| .
$$

Since $s_{R}-s_{Q}=h^{\prime}+Q D \tan \theta$ and $Q D=\left(s_{R}-s_{Q}\right) \tan \theta_{o}$, by (5.21) we have

$$
s_{R}-s_{Q}=\frac{h^{\prime}}{1-\tan \theta \tan \theta_{o}} \leq \frac{\tan \theta-\tan \theta_{o}}{1-\tan \theta \tan \theta_{o}}\left|\delta_{1}^{\prime}\right| .
$$

Clearly, $\frac{\tan \theta-\xi}{1-\xi \tan \theta}$ is a decreasing function of $\xi \in[0, \tan \theta]$ and then $s_{R}-s_{Q} \leq$ $\left|\delta_{1}^{\prime}\right| \tan \theta$. This proves (5.18) with $c_{L R}=\tan \theta<1$. At last, $k_{o}\left|\varepsilon_{3}\right|=\left|\varepsilon_{1}\right|-\left|\delta_{1}\right|-\left|\delta_{1}^{\prime}\right|$, whence (5.19).

Remark 5.5. By the proof of Proposition 5.4 we see that $c_{L R} \leq f_{o}^{\prime}\left(\delta_{i}\right)$ in cases (2)-(3) and $c_{L R} \leq f_{o}^{\prime}\left(\delta_{i}+\delta_{i}^{\prime}-k_{o} l_{o}\left|\delta_{i} \delta_{i}^{\prime}\right|\right)$ in case (4), where $\delta_{i}$ and $\delta_{i}^{\prime}$ are the strengths of the interacting shocks. The estimate (5.18) may be considered as an improvement of 2 , $(5.20)]$.

The dependence of the constant $c_{L R}$ on the waves involved in the interaction can be made uniform not only with respect to the states $(v, u)$ but also with respect to $\lambda$. The bounds in Remark 5.5] show in fact that $c_{L R}$ depends implicitly also on $\lambda_{o}$, then on $a_{*}$, $a^{*}$; see (3.1).

A uniform expression is achieved by arguing as in [19] or [1]. Indeed, fix a positive number $M>0$, to be defined later on in (11.43), and assume that

$$
\text { the strength of any interacting shock wave is less than } M \text {. }
$$

Then there exists $c=c\left(M, a_{*}, a^{*}\right) \in(0,1)$ (for simplicity the dependence of $c$ on $M, a_{*}, a^{*}$ shall be dropped in the following) such that

$$
\left|\varepsilon_{j}\right| \leq \begin{cases}c \cdot \zeta_{i} & \text { in case (2), } \\ c \cdot\left|\delta_{i}\right| & \text { in case (3), } \\ \min \left\{c\left|\delta_{i}\right|, c\left|\delta_{i}^{\prime}\right|, l_{o}\left|\delta_{i} \delta_{i}^{\prime}\right|\right\} & \text { in case (4). }\end{cases}
$$

The function $f_{o}(x)$, defined in the proof of Lemma 3.1, in fact depends on $\lambda_{o}$ only through $a_{o}$ and we denote it by $f(x, a)$, replacing $a_{o}$ with $a$. Recalling Remark 5.5 we find an upper bound for $c\left(M, a_{*}, a^{*}\right)$ :

$$
\begin{aligned}
& c\left(M, a_{*}, a^{*}\right) \\
& \quad \leq \max \left\{\max _{\substack{a * \leq a \leq a^{*} \\
-M \leq \delta \leq 0}} f_{x}(-\delta, a), \max _{\substack{a_{*} \leq a \leq a^{*} \\
-M \leq \delta, \delta^{\prime} \leq 0}} f_{x}\left(-\delta-\delta^{\prime}-\frac{a-a_{*}}{2\left(a+a_{*}\right)^{2}} \delta \delta^{\prime}, a\right)\right\} .
\end{aligned}
$$

Remark 5.6. Consider case (4) in Proposition 5.4. For these interactions, and as far as the size of $\varepsilon_{j}$ is concerned, we can assume

$$
l_{o} \max \left\{\left|\delta_{i}\right|,\left|\delta_{i}^{\prime}\right|\right\}<c .
$$


Then, the following weaker estimate holds:

$$
\left|\varepsilon_{j}\right| \leq c \min \left\{\left|\delta_{i}\right|,\left|\delta_{i}^{\prime}\right|\right\}
$$

In order to define the decomposition of paths we summarize in the following proposition what we proved in Sections 5.1 and 5.2 on shocks produced by physical interactions.

Proposition 5.7. Consider an interaction between physical waves, under the notation of Propositions 5.2, 5.4 and assume (5.22). Then there exist positive numbers $h^{\prime}$ and $h^{\prime \prime}$, possibly changing from line to line, such that:

2 int's:

(iii) $\left|\varepsilon_{i}\right| \leq\left|\delta_{i}\right|$

(iv) $\left|\varepsilon_{i}\right|=\left|\delta_{i}\right|+h^{\prime \prime}\left|\delta_{2} \delta_{i}\right|$

13 int's:
(2) $\quad\left|\varepsilon_{i}\right|=\left|\delta_{i}\right|-\zeta_{i}$

(4) $\left|\varepsilon_{i}\right|=\left|\delta_{i}\right|+\left|\delta_{i}^{\prime}\right|+h^{\prime \prime}\left|\delta_{i} \delta_{i}^{\prime}\right|$

$$
\begin{array}{ll}
\left|\varepsilon_{j}\right|=h^{\prime \prime}\left|\delta_{2} \delta_{i}\right|, & h^{\prime \prime} \leq \frac{1}{2\left(1-[a]_{*}\right)} \\
\left|\varepsilon_{j}\right|=h^{\prime \prime}\left|\delta_{2} \delta_{i}\right|, & h^{\prime \prime} \leq 1 / 2 \\
& h^{\prime \prime} \leq 1 / 2
\end{array}
$$

$\left|\varepsilon_{j}\right|=h^{\prime} \zeta_{i}, \quad h^{\prime} \leq c$

$\left|\varepsilon_{j}\right|=h^{\prime}\left|\delta_{i}\right|, \quad h^{\prime} \leq c$

$h^{\prime \prime} \leq k_{o} l_{o}$.

6. The front-tracking algorithm. In this section we define the front-tracking algorithm that is used to construct piecewise constant approximate solutions; we refer to [7] for more information.

First we approximate the initial data $\bar{U}=(\bar{v}, \bar{u}, \bar{\lambda})$ with a sequence of piecewise constant functions $\bar{U}^{\nu}=\left(\bar{v}^{\nu}, \bar{u}^{\nu}, \bar{\lambda}^{\nu}\right), \nu \in \mathbb{N}$, with a finite number of jumps, such that

$$
\begin{gathered}
\operatorname{TV}\left(\bar{v}^{\nu}\right) \leq \operatorname{TV}(\bar{v}), \bar{v}^{\nu} \geq v_{0}, \operatorname{TV}\left(\bar{u}^{\nu}\right) \leq \operatorname{TV}(\bar{u}), \operatorname{WTV}\left(a\left(\bar{\lambda}^{\nu}\right)\right) \leq \operatorname{WTV}(a(\bar{\lambda})) ; \\
\bar{a}_{*} \leq \inf _{x \in \mathbb{R}} a\left(\bar{\lambda}^{\nu}(x)\right), \quad \sup _{x \in \mathbb{R}} a\left(\bar{\lambda}^{\nu}(x)\right) \leq \bar{a}^{*} ; \\
\lim _{x \rightarrow \pm \infty}\left(\bar{v}^{\nu}, \bar{u}^{\nu}, \bar{\lambda}^{\nu}\right)(x)=\lim _{x \rightarrow \pm \infty}(\bar{v}, \bar{u}, \bar{\lambda})(x) \\
\left\|\left(\bar{v}^{\nu}, \bar{u}^{\nu}, \bar{\lambda}^{\nu}\right)-(\bar{v}, \bar{u}, \bar{\lambda})\right\|_{\mathbf{L}^{1}} \leq \frac{1}{\nu} .
\end{gathered}
$$

Fix parameters $\eta=\eta_{\nu}>0, \rho=\rho_{\nu}>0$ which vanish for $\nu \rightarrow \infty$ and let $\hat{s}$ be a positive number strictly larger than the speed of any wave; all three parameters are specified later on. As in [7] we use two approximate Riemann solvers that we briefly recall. We denote by $\mathcal{N} \mathcal{P}$ the set of nonphysical waves.

- The accurate Riemann solver is obtained by replacing any rarefaction wave in the exact solution given in Section 4 by a centered rarefaction fan; if the size of the rarefaction wave is $\sigma$, then the fan contains $N=[\sigma / \eta]+1$ fronts and each of them has strength $\sigma / N<\eta$.

- Assume two wave-fronts of sizes $\sigma, \tau$ interact. The simplified Riemann solver consists in prolonging each wave with a wave of the same family and size; if the fronts belong to the same family, they are prolonged as a single front of size $\sigma+\tau$. Since waves in general do not commute, a nonphysical front is introduced with speed $\hat{s}$. Its size is defined as in [7]. The interaction of a physical front with a nonphysical front is solved by prolonging the physical front with a front of the same family and size and by a nonphysical front.

An approximate solution is constructed as follows. 
(1) At any jump point of $U_{o}^{\nu}$ we solve the Riemann problem using the accurate Riemann solver. This gives an approximate solution $U^{\nu}(x, t)$ for $x \in \mathbb{R}$ which is well defined until a time $t_{1}>0$ is reached, when a first set of interactions takes place.

(2) By changing possibly the speeds of some waves of a quantity $O(1) / \nu$ we can assume that only two fronts interact at a time. At time $t_{1}$ we solve again the Riemann problem in an approximate way and extend $U^{\nu}$ until the next interaction time $t_{2}$, and so on. These interactions are solved by using the accurate Riemann solver if the sizes $\sigma, \tau$ of the physical interacting fronts satisfy $|\sigma \tau| \geq \rho$, the simplified Riemann solver if $|\sigma \tau|<\rho$; if one of the interacting fronts is a nonphysical front we use the simplified Riemann solver.

In order to prove that $U^{\nu}$ is well defined for $t \in(0,+\infty)$ we show in Section 11 that the number of interactions remains finite for any time. Observe that this scheme differs from that in [2]: there the interaction of 1 - and 3-waves was always solved with the accurate Riemann solver.

Now, we introduce the functionals. Consider any time $t>0$ when interactions do not occur and $i=1,3$. We denote by $S_{i}(t)\left(R_{i}(t)\right)$ the set of $i$-shock (resp., rarefaction) waves at time $t$ and by $C D$ the set of contact discontinuities in the approximate solution. By $\mathcal{A}(t)$ we denote the set of all approaching pairs of waves $\left(\delta_{2}, \delta_{i}\right)$, for $\delta_{2} \in C D(t)$ and $\delta_{i}$ any wave (shock or rarefaction) of the $i$ family at time $t$ : so either $i=1$ and $\delta_{1}$ is on the right of $\delta_{2}$ or $i=3$ and $\delta_{3}$ is on the left of $\delta_{2}$. We define, for positive constants $K$ and $K_{2}$ to be specified later on,

$$
\begin{array}{rlrlrl}
L(t) & =\sum_{\delta_{i} \in S_{i}(t), i=1,3}\left|\delta_{i}\right|, & Q(t) & = & \sum_{\delta_{i}, \delta_{i}^{\prime} \in S_{i}(t), i=1,3}\left|\delta_{i} \delta_{i}^{\prime}\right|, \\
L^{+}(t) & =\sum_{\delta_{i} \in R_{i}(t), i=1,3}\left|\delta_{i}\right|, & Q_{2}(t) & = & \sum_{\left(\delta_{2}, \delta_{i}\right) \in \mathcal{A}(t), i=1,3}\left|\delta_{2} \delta_{i}\right|, \\
L_{\text {tot }}(t) & =L(t)+L^{+}(t), & & \mathcal{Q}(t) & = & K Q(t)+K_{2} Q_{2}(t), \\
L_{2} & =\sum_{\delta_{2} \in C D}\left|\delta_{2}\right|, & F(t) & = & L(t)+K Q(t)+K_{2} Q_{2}(t) .
\end{array}
$$

We also denote for $i=1,3$ the variation of the shocks of the family $i$ by

$$
L_{i}(t)=\sum_{\delta_{i} \in S_{i}(t)}\left|\delta_{i}\right|
$$

The strengths of the contact discontinuities do not change by the wave interactions and then $L_{2}$ is a constant. Moreover,

$$
L_{2} \geq 2[\bar{a}]_{*}
$$

because of the estimate $\operatorname{WTV}(f) \geq 2 \frac{|f(x)-f(y)|}{f(x)+f(y)}$, which holds true for every $f: \mathbb{R} \rightarrow$ $(0,+\infty)$ and $x, y \in \mathbb{R}$. Notice that $L_{2}=2[\bar{a}]_{*}$ in the case of a single 2 -wave. If a contact discontinuity $\delta_{2}$ is fixed and there is no ambiguity of notation we write $L_{2}^{l}=\sum\left|\delta_{2}^{\prime}\right|$ for $\delta_{2}^{\prime}$ on the left of $\delta_{2}$ and $L_{2}^{r}$ analogously.

Observe that the functional $L$ accounts only for shock waves, as in [18, [19] and differently than in [7, 2]. On the other hand, we considered in $Q$ only products of shock 
waves of the same family, differently from [19] where only shocks of different family were introduced; the reason is that in our case shocks of different families interact without changing their strengths. We denote $\Delta L(t)=L(t+)-L(t-)$, and so on.

With an abuse of notation, for any interaction point $\mathrm{P}$ we denote by $Q(\mathrm{P}), Q_{2}(\mathrm{P})$ the interaction potentials restricted at the interaction at $\mathrm{P}$. More precisely, $Q(\mathrm{P})$ equals $\left|\delta_{i} \delta_{i}^{\prime}\right|$ if two shocks $\delta_{i}, \delta_{i}^{\prime}$ interact at $\mathrm{P}$ and 0 otherwise; $Q_{2}(\mathrm{P})$ equals $\left|\delta_{2} \delta_{i}\right|$ if an $i$-wave interacts with the 2 -wave $\delta_{2}$ at $\mathrm{P}$. We always write these potentials by expressing their dependence on $\mathrm{P}$; no confusion with the potentials above is possible.

7. Estimates on the functionals. Here we study the variations at single interactions of the functionals introduced in Section 6. Because of (6.1), we apply the estimates of Propositions 5.2 and 5.4 with $a_{*}=\bar{a}_{*}$ and $a^{*}=\bar{a}^{*}$. Consequently, with a slight abuse of notation, we now write

$$
k_{o}=\frac{a_{o}-\bar{a}_{*}}{a_{o}+\bar{a}_{*}} \quad \text { and } \quad l_{o}=\frac{1}{2\left(a_{o}+\bar{a}_{*}\right)} .
$$

Moreover, we introduce

$$
K_{0} \doteq \frac{1}{4 \bar{a}_{*}}
$$

Notice that $k_{o} \leq[\bar{a}]_{*}$ and $l_{o} \leq K_{0}$.

Proposition 7.1. Assume that at time $t$ a 1 - or a 3 -wave of strength $\delta_{i}$ interacts with a 2-wave of strength $\delta_{2}$. Then

- in case CD1:

$$
\Delta L \leq \frac{1}{2}\left|\delta_{2} \delta_{i}\right|, \quad \Delta Q \leq \frac{1}{2}\left|\delta_{2} \delta_{i}\right| L(t-), \quad \Delta Q_{2} \leq \frac{1}{2}\left|\delta_{2} \delta_{i}\right|\left(L_{2}-2\right) ;
$$

- in case CD2:

$$
\begin{gathered}
\Delta L \leq \frac{1}{2} \frac{1}{1-[\bar{a}]_{*}}\left|\delta_{2} \delta_{i}\right|, \Delta Q \leq \frac{1}{2} \frac{1}{1-[\bar{a}]_{*}}\left|\delta_{2} \delta_{i}\right| L(t-), \\
\Delta Q_{2} \leq \frac{1}{2} \frac{1}{1-[\bar{a}]_{*}}\left|\delta_{2} \delta_{i}\right|\left(L_{2}-2\left(1-[\bar{a}]_{*}\right)\right) .
\end{gathered}
$$

Proof. Consider the proof of Proposition 5.2. In case (i) we have $\Delta L=\Delta Q=0$. In case (ii) we have $\Delta L=\left|\varepsilon_{j}\right| \leq \frac{1}{2} \frac{1}{1-[\bar{a}]_{*}}\left|\delta_{2} \delta_{i}\right|$ and $\Delta Q=\left|\varepsilon_{j}\right| L_{j}(t-) \leq \frac{1}{2} \frac{1}{1-[\bar{a}]_{*}}\left|\delta_{2} \delta_{i}\right| L(t-)$.

In case (iii) we have $\Delta L=\left|\varepsilon_{1}\right|+\left|\varepsilon_{3}\right|-\left|\delta_{i}\right|=-\left|\delta_{i}-\varepsilon_{i}\right|+\left|\varepsilon_{j}\right| \leq\left|\delta_{2} \delta_{i}\right| / 2$ and $\Delta Q=$ $\left(\left|\varepsilon_{i}\right|-\left|\delta_{i}\right|\right) L_{i}(t-)+\left|\varepsilon_{j}\right| L_{j}(t-) \leq(1 / 2)\left|\delta_{2} \delta_{i}\right| L(t-)$ because $\left|\varepsilon_{i}\right|-\left|\delta_{i}\right|<0$.

In case (iv) $\Delta L=\left|\varepsilon_{i}\right|-\left|\delta_{i}\right|=\left|\delta_{i}-\varepsilon_{i}\right| \leq\left|\delta_{2} \delta_{i}\right| / 2, \Delta Q=\left(\left|\varepsilon_{i}\right|-\left|\delta_{i}\right|\right) L_{i}(t-) \leq$ $(1 / 2)\left|\delta_{2} \delta_{i}\right| L(t-)$.

At last, the estimate for $\Delta Q_{2}$ follows from $\Delta Q_{2}=-\left|\delta_{2} \delta_{i}\right|+\left(\left|\varepsilon_{i}\right|-\left|\delta_{i}\right|\right) L_{2}^{l, r}+\left|\varepsilon_{j}\right| L_{2}^{r, l}$, where

and analogously $L_{2}^{r, l}$.

$$
L_{2}^{l, r}= \begin{cases}L_{2}^{l} & \text { if } i=1 \\ L_{2}^{r} & \text { if } i=3\end{cases}
$$

Proposition 7.2. Consider an $i$-wave $W_{i}$ and a $j$-wave $W_{j}, i, j \in\{1,3\}$, which interact at time $t>0$. Under the notation of Proposition 5.4 we have the following: 
(1) $W_{3} W_{1} \rightarrow W_{1} W_{3}: \Delta L=\Delta Q=\Delta Q_{2}=0$;

(2) $S_{i} R_{i}^{\prime} \rightarrow S_{i} S_{j}: \Delta L \leq-\left(1-c_{L R}\right) \zeta_{i}, \Delta Q \leq c_{L R} L(t-) \zeta_{i}, \Delta Q_{2} \leq c_{L R} L_{2} \zeta_{i}$;

(3) $S_{i} R_{i}^{\prime} \rightarrow R_{i} S_{j}: \Delta L \leq-\left(1-c_{L R}\right)\left|\delta_{i}\right|, \Delta Q \leq c_{L R} L(t-)\left|\delta_{i}\right|, \Delta Q_{2} \leq c_{L R} L_{2}\left|\delta_{i}\right|$;

(4) $S_{i} S_{i}^{\prime} \rightarrow S_{i} R_{j}: \Delta L \leq k_{o} l_{o}\left|\delta_{i} \delta_{i}^{\prime}\right|, \Delta Q \leq-\left(1-k_{o} l_{o} L(t-)\right)\left|\delta_{i} \delta_{i}^{\prime}\right|$ and $\Delta Q_{2} \leq l_{o} L_{2}\left|\delta_{i} \delta_{i}^{\prime}\right|$.

Proof. We consider Proposition 5.4, all estimates on $\Delta L$ follow in a straightforward way. Therefore we deal only with the estimates for $\Delta Q$ and $\Delta Q_{2}$; in case (1) there is nothing to prove.

(2) Using the notation introduced in (17.2) we have $\Delta Q=\left(\left|\varepsilon_{i}\right|-\left|\delta_{i}\right|\right) L_{i}(t-)+\left|\varepsilon_{j}\right| L_{j}(t-) \leq$ $c_{L R} L(t-) \zeta_{i}$ and $\Delta Q_{2}=\left(\left|\varepsilon_{i}\right|-\left|\delta_{i}\right|-\left|\delta_{i}^{\prime}\right|\right) L_{2}^{l, r}+\left|\varepsilon_{j}\right| L_{2}^{r, l} \leq c_{L R} L_{2} \zeta_{i}$.

(3) We have $\Delta Q=\left|\varepsilon_{j}\right| L_{j}(t-)-\left|\delta_{i}\right| L_{i}(t-) \leq c_{L R} L(t-)\left|\delta_{i}\right|$ and $\Delta Q_{2}=\left(\left|\varepsilon_{i}\right|-\left|\delta_{i}\right|-\right.$ $\left.\left|\delta_{i}^{\prime}\right|\right) L_{2}^{l, r}+\left|\varepsilon_{j}\right| L_{2}^{r, l} \leq c_{L R} L_{2}\left|\delta_{i}\right|$.

(4) At last we have $\Delta Q \leq-\left|\delta_{i} \delta_{i}^{\prime}\right|+k_{o} l_{o}\left|\delta_{i} \delta_{i}^{\prime}\right| L(t-)=-\left(1-k_{o} l_{o} L(t-)\right)\left|\delta_{i} \delta_{i}^{\prime}\right|$ and $\Delta Q_{2} \leq$ $k_{o} l_{o}\left|\delta_{i} \delta_{i}^{\prime}\right| L_{2}^{l, r}+l_{o}\left|\delta_{i} \delta_{i}^{\prime}\right| L_{2}^{r, l} \leq l_{o} L_{2}\left|\delta_{i} \delta_{i}^{\prime}\right|$.

REMARK 7.3. In an interaction with a 2 -wave, both $L$ and $Q$ may increase while $Q_{2}$ decreases if

$$
L_{2}<2\left(1-[\bar{a}]_{*}\right) \doteq D .
$$

In an interaction between 1- and 3-waves the functional $Q_{2}$ may increase; also the functional $L$ may increase, but if this happens (in case (4) above), then $Q$ decreases if

$$
K_{0} L(t-)<\frac{1}{[\bar{a}]_{*}} \doteq A .
$$

Observe that for interactions of 1- and 3-waves of different families, the accurate and the simplified Riemann solver coincide; no nonphysical wave is emitted.

REMARK 7.4. The term $\Delta L^{+}$is always controlled by a quadratic term. Indeed, in case (i): $\Delta L^{+} \leq\left|\delta_{2} \delta_{i}\right|$; case (ii): $\Delta L^{+} \leq \frac{1}{2\left(1-[\bar{a}]_{*}\right)}\left|\delta_{2} \delta_{i}\right|$; case (iv): $\Delta L^{+} \leq \frac{1}{2}\left|\delta_{2} \delta_{i}\right|$; case (1): $\Delta L^{+}=0$; case (3): $\Delta L^{+} \leq 0$; case (4): $\Delta L^{+} \leq l_{o}\left|\delta_{i} \delta_{i}^{\prime}\right|$. This proves our claim.

8. Local and global decreasing of the functional $F$. We assume that the approximate solution constructed in Section [6 is defined in $[0, T)$ for some $T \in(0,+\infty]$. The key condition (5.22), which is always assumed throughout the following, is intended to hold for any shock wave in the approximate solution, at any time. As in the beginning of Section 7 , here we still denote $c=c\left(M, \bar{a}_{*}, \bar{a}^{*}\right) \in(0,1)$. We assume $[\bar{a}]_{*}>0$; if $[\bar{a}]_{*}=0$, then system (1.1) reduces to the $p$-system.

If $t<T$ is an interaction time, one could guess by Remark 7.3 that $L_{2}<D$ and $K_{0} L(t-)<A$ imply $\Delta F(t)<0$. We prove below that the functional $F$ is decreasing under the slightly stronger conditions

$$
\begin{aligned}
L_{2} & <D \cdot \frac{1-c}{2-c} \\
K_{0} L(t-) & <A \cdot \frac{D(1-c)-(2-c) L_{2}}{(2-c)\left\{(A-1) L_{2}+D\right\}} \doteq B .
\end{aligned}
$$

Under the notation of Section 2 the condition (8.1) reads $\langle\bar{a}\rangle\left\langle\frac{1-c}{2-c}\right.$, which holds as a consequence of (2.6) because $\langle\bar{a}\rangle \leq\langle\bar{a}\rangle \mathrm{e}^{\langle\bar{a}\rangle}$ and $\frac{1}{2} \frac{(1-c)^{2}}{2-c}<\frac{1-c}{2-c}$. Nevertheless, in the 
following we shall refer to the weaker condition (8.1) until (2.6) is really needed; see Proposition 11.8, Observe that (8.1) is compatible with (6.4) if and only if

$$
[\bar{a}]_{*}<\frac{1-c}{3-2 c}
$$

holds. If (8.3) does not hold, then (8.1), and then also (2.6), fails, a contradiction. The bound $(8.3)$ for $[\bar{a}]_{*}$ is a decreasing function of $c$, and $0<[\bar{a}]_{*}<1 / 3$.

Indeed, $F$ decreases under assumptions weaker than (8.1) and (8.2), which however are not sufficient to prove the stability of the algorithm. Moreover, we have the estimates $B<A, 2-D<L_{2}$ (because of (ㅎ․4) ) and

$$
\frac{2(2-c)}{3-2 c}<D<2<\frac{3-2 c}{1-c}<A .
$$

We also note that $B \rightarrow \infty$ as $L_{2} \rightarrow 0$, since

$$
B=\frac{2 D(1-c)-2(2-c) L_{2}}{(2-c) D\left(L_{2}+2-D\right)}=\frac{2\left(1-[\bar{a}]_{*}\right)(1-c)-(2-c) L_{2}}{(2-c)\left(1-[\bar{a}]_{*}\right)\left(L_{2}+2[\bar{a}]_{*}\right)} .
$$

At last, denote for short $L=L(t-)$ and define

$$
\mathcal{D} \doteq\left(A-K_{0} L\right)\left(D-L_{2}\right)-A K_{0} L L_{2} .
$$

We have $\mathcal{D}>0$, since it is equivalent to $K_{0} L<A \cdot \frac{D-L_{2}}{D+(A-1) L_{2}}$, which holds true because of $(8.2)$.

Proposition 8.1. Assume (5.22). Let $t<T$ be an interaction time of two physical waves, denote $L=L(t-)$ and assume (8.1), (8.2).

Then, for every $K_{2}$ satisfying

$$
\frac{A}{\mathcal{D}} \leq K_{2} \leq \frac{1}{L_{2}} \cdot \frac{(1-c) A-(2-c) K_{0} L}{A-K_{0} L+A K_{0} L}
$$

we can find $K$, with

$$
\frac{K_{0}\left(A L_{2} K_{2}+1\right)}{A-K_{0} L} \leq K \leq \frac{1}{L} \min \left\{\left(D-L_{2}\right) K_{2}-1,1-c-L_{2} K_{2}\right\},
$$

such that

$$
\Delta F(t) \leq 0 .
$$

Proof. We study the variation $\Delta F$ at different interactions of physical waves; in the case $W_{3} W_{1} \rightarrow W_{1} W_{3}$ we have clearly $\Delta F=0$.

- $W_{i} C_{2}$. By Proposition 7.1 in case $C D 1$ we have

$$
\Delta F \leq \frac{1}{2}\left|\delta_{2} \delta_{i}\right| \cdot\left(1+K L-K_{2}\left(2-L_{2}\right)\right) \leq 0
$$

if $K \leq \frac{K_{2}\left(2-L_{2}\right)-1}{L}$, while in case $C D 2$ we have

$$
\Delta F \leq \frac{1}{2\left(1-[\bar{a}]_{*}\right)}\left|\delta_{2} \delta_{i}\right| \cdot\left(1+K L-K_{2}\left(2\left(1-[\bar{a}]_{*}\right)-L_{2}\right)\right) \leq 0
$$

if

$$
K \leq \frac{\left(D-L_{2}\right) K_{2}-1}{L} .
$$

Observe that $\frac{\left(D-L_{2}\right) K_{2}-1}{L}<\frac{K_{2}\left(2-L_{2}\right)-1}{L}$; then $\Delta F(t) \leq 0$ in both cases under (8.8). 
- $S_{i} R_{i}^{\prime} \rightarrow S_{i} S_{j}$. From Proposition 7.2 we have

$$
\Delta F \leq\left\{-(1-c)+c K L+c K_{2} L_{2}\right\} \zeta_{i} \leq 0
$$

if

$$
K \leq \frac{1}{L}\left(\frac{1-c}{c}-L_{2} K_{2}\right)
$$

In Section 11, in order to prove the stability of the front-tracking scheme, we shall need the condition

$$
K L+K_{2} L_{2}<1-c,
$$

which is stronger than (8.10). Therefore we already require here (8.11) instead of (8.10).

- $S_{i} R_{i}^{\prime} \rightarrow R_{i} S_{j}$. By (8.10) we find $\Delta F \leq\left\{-(1-c)+c K L+c K_{2} L_{2}\right\}\left|\delta_{i}\right|<0$.

- $S_{i} S_{i}^{\prime} \rightarrow S_{i} R_{j}$. In this case we have

$$
\Delta F \leq\left(K_{0}[\bar{a}]_{*}-K\left(1-K_{0}[\bar{a}]_{*} L\right)+K_{0} K_{2} L_{2}\right) \cdot\left|\delta_{i} \delta_{i}^{\prime}\right| \leq 0
$$

if $-K\left(1-K_{0}[\bar{a}]_{*} L\right)+K_{0} K_{2} L_{2} \leq-K_{0}[\bar{a}]_{*}$, which is equivalent to

$$
K \geq \frac{K_{0}\left(A K_{2} L_{2}+1\right)}{A-K_{0} L} .
$$

Now, we must check whether the inequalities (8.8), (8.11) and (8.13) admit positive solutions $K_{2}, K$ in the plane $\left(K_{2}, K\right)=(x, y)$; refer to Figure 7 (a). The inequalities (8.8) and (8.13) are equivalent to

$$
\frac{K_{0}\left(A L_{2} K_{2}+1\right)}{A-K_{0} L} \leq K \leq \frac{\left(D-L_{2}\right) K_{2}-1}{L} .
$$

Condition $\mathcal{D}>0$ requires precisely that the slope of the boundary line of (8.8) is strictly larger than that of (8.13). Let $\mathrm{P}_{1}=\left(x_{1}, y_{1}\right)=\frac{1}{\mathcal{D}}\left(A, K_{0}\left(D-L_{2}+A L_{2}\right)\right)$ denote the intersection point of these boundary lines. Then we can find $K$ satisfying (8.14) if $K_{2} \geq x_{1}$.

Let $\mathrm{P}_{2}=\left(x_{2}, y_{2}\right)$, with $x_{2}=\frac{2-c}{D}, y_{2}=\frac{D(1-c)-(2-c) L_{2}}{D L}$, denote the intersection point of the boundary lines of (8.8) and (8.11). Now, $K_{2}=x_{1}, K=y_{1}$ must satisfy (8.11). Such a condition is satisfied in a strict sense iff $\frac{A D}{\mathcal{D}}<2-c$; in turn, this last condition is equivalent to (8.2).

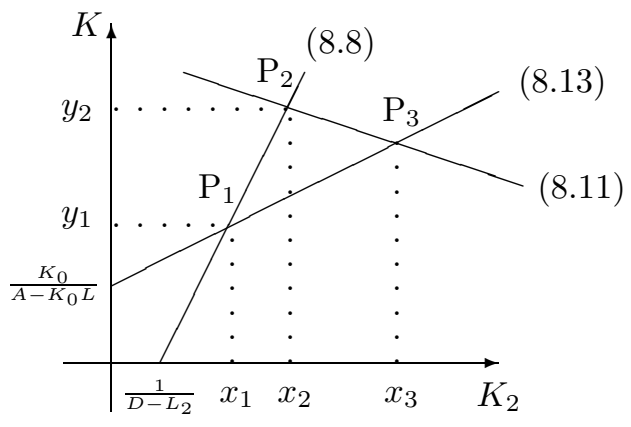

(a)

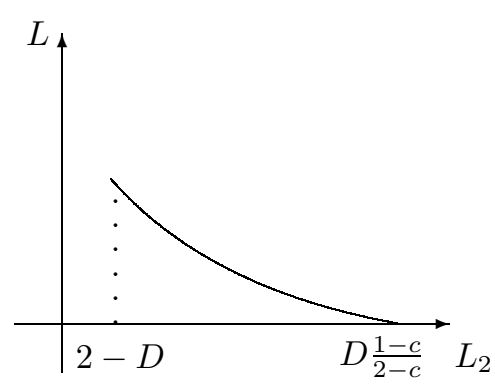

(b)

FIG. 7. (a): choice of the parameters $K, K_{2}$. (b): range of $L_{2}, L$; the solid line is the graph of the function $L=B\left(L_{2}\right) / K_{0}$. 
Let $\mathrm{P}_{3}=\left(x_{3}, y_{3}\right)$, with $x_{3}=\frac{1}{L_{2}} \cdot \frac{(1-c) A-(2-c) K_{0} L}{A-K_{0} L+A K_{0} L}$, be the intersection point of the boundary lines of (8.13) and (8.11). This gives the inequality on the right in (8.4).

We deal now with the problem of finding conditions on the initial data in order that (8.2) is satisfied for every $t \geq 0$. Note that the value $x_{2}$ in the proof above is independent of both $L$ and $L_{2}$. We fix $K_{2}=\frac{2-c}{D}=x_{2}$; as a consequence, (8.5) reads

$$
\frac{K_{0}\left\{A L_{2}(2-c)+D\right\}}{D\left(A-K_{0} L\right)} \leq K \leq \frac{D(1-c)-(2-c) L_{2}}{D L}=y_{2}\left(L_{2}, L\right) .
$$

The above left (right) term is an increasing (decreasing) function of $L$. Observe that the term $B$ does not depend on $t$ but only on the initial data; it is a decreasing function of $L_{2}$; see Figure 7(b). As $L$ increases up to $K_{0} L=B$, the interval (8.15) shrinks to the point $y_{2}\left(L_{2}, B / K_{0}\right)=\frac{K_{0}(2-c)\left\{(A-1) L_{2}+D\right\}}{A D}$; we thus fix $K_{2}=y_{2}\left(L_{2}, B / K_{0}\right)$. We have proved the following result.

Proposition 8.2. If we set

$$
K_{2}=\frac{2-c}{2\left(1-[\bar{a}]_{*}\right)}, \quad K=\frac{K_{0}(2-c)\left(L_{2}+2[\bar{a}]_{*}\right)}{2},
$$

then the condition (8.15) holds for all $0<L<B / K_{0}$. Moreover, $K \rightarrow 0$ as $L_{2} \rightarrow 0$.

Corollary 8.3. Under (5.22) choose $K$ and $K_{2}$ as in (8.16); moreover, assume

$$
L_{\text {tot }}(0)<\frac{B}{(2-c) K_{0}} .
$$

Then for every $t \in[0, T)$,

$$
L(t) \leq F(0)<\frac{B}{K_{0}} .
$$

Proof. Condition (8.17) implies (8.2) and then (8.11) with 0 instead of $t$-. As a consequence,

$$
F(0) \leq L(0)+K L(0)^{2}+K_{2} L_{2} L_{\text {tot }}(0) \leq \frac{B}{(2-c) K_{0}}\left(1+K L(0)+K_{2} L_{2}\right) \leq \frac{B}{K_{0}} .
$$

Then, we argue by induction on the interaction times $t_{k}, k=1,2, \ldots$ : Proposition 8.1 applies with $t=t_{k}$ and gives $L\left(t_{k}+\right) \leq F\left(t_{k}+\right) \leq F\left(t_{k}-\right) \leq F(0+)<B / K_{0}$.

A consequence of the above arguments is that, if we assume (5.22), (8.1), (8.17) and choose $K, K_{2}$ as in Proposition 8.2. then Corollary 8.3 and Proposition 8.1 imply $\Delta F(t) \leq 0$. Define

$$
\left(\bar{v}_{ \pm \infty}, \bar{u}_{ \pm \infty}\right)=(\bar{v}( \pm \infty), \bar{u}( \pm \infty)) .
$$

By taking into account (6.2) and (A.5) we deduce

$$
\begin{aligned}
\left|(v(x, t), u(x, t))-\left(\bar{v}_{-\infty}, \bar{u}_{-\infty}\right)\right| & \leq \operatorname{TV}(v(\cdot, t), u(\cdot, t)) \\
& \leq 2\left|\left(\bar{v}_{+\infty}, \bar{u}_{+\infty}\right)-\left(\bar{v}_{-\infty}, \bar{u}_{-\infty}\right)\right|+3 F(t) \\
& \leq 2\left|\left(\bar{v}_{+\infty}, \bar{u}_{+\infty}\right)-\left(\bar{v}_{-\infty}, \bar{u}_{-\infty}\right)\right|+3 F(0),(8.20)
\end{aligned}
$$

where we used the notation in (3.3).

Now, we give some estimates which refine those in Proposition 8.1 in the cases $W_{i} C_{2}$ and $S_{i} S_{i}^{\prime}$. 
Proposition 8.4. Under the assumptions of Corollary 8.3, consider a sequence of interaction points $\left\{\mathrm{P}_{m}\right\}=\left\{\left(x_{m}, t_{m}\right)\right\}$, with $t_{m}<t_{m+1}<T$ for $m=1,2, \ldots$, where either an $i$-wave interacts with a 2 -wave $\left(\right.$ case $W_{i} C_{2}$ ) or two shock waves of the same family interact (case $S_{i} S_{i}^{\prime}$ ). Then

$$
\begin{aligned}
\frac{1}{2\left(1-[\bar{a}]_{*}\right)}\left|\delta_{2} \delta_{i}\right| \leq-\left(K \Delta Q+K_{2} \Delta Q_{2}\right), & \text { in case } W_{i} C_{2}, \\
{[\bar{a}]_{*} K_{0} \mid \delta_{i} \delta_{i}^{\prime} \leq-\left(K \Delta Q+K_{2} \Delta Q_{2}\right), } & \text { in case } S_{i} S_{i}^{\prime},
\end{aligned}
$$

and

$$
\sum_{m}\left\{[\bar{a}]_{*} K_{0} Q\left(t_{m}-\right)+\frac{1}{2\left(1-[\bar{a}]_{*}\right)} Q_{2}\left(t_{m}-\right)\right\} \leq K Q(0)+K_{2} Q_{2}(0) .
$$

Proof. In case $W_{i} C_{2}$ by Proposition 7.1 we have

$$
K \Delta Q+K_{2} \Delta Q_{2} \leq \frac{1}{2} \frac{1}{1-[\bar{a}]_{*}}\left|\delta_{2} \delta_{i}\right|\left(K L+K_{2}\left(L_{2}-2\left(1-[\bar{a}]_{*}\right)\right)\right)
$$

and from (8.7) we deduce $1<-\left(K L-K_{2}\left(2\left(1-[\bar{a}]_{*}\right)-L_{2}\right)\right)$. If we multiply both sides of this inequality by $\frac{\left|\delta_{2} \delta_{i}\right|}{2\left(1-[\bar{a}]_{*}\right)}$ and make use of (8.24) we get (8.21).

In case $S_{i} S_{i}^{\prime}$ by Proposition 7.2, case (4), we have

$$
K \Delta Q+K_{2} \Delta Q_{2} \leq\left|\delta_{i} \delta_{i}^{\prime}\right|\left(-K\left(1-[\bar{a}]_{*} K_{0} L\right)+K_{2} K_{0} L_{2}\right) .
$$

By (8.12) we have $-K\left(1-[\bar{a}]_{*} K_{0} L\right)+K_{0} K_{2} L_{2} \leq-[\bar{a}]_{*} K_{0}$; if we multiply both sides of this inequality by $\left|\delta_{i} \delta_{i}^{\prime}\right|$ and make use of (8.25), we obtain (8.22).

At last, if for some $m$ there occur interactions in $\left(t_{m}, t_{m+1}\right)$, we add such interaction points to the sequence $\left\{\mathrm{P}_{m}\right\}$ and get a sequence $\left\{\mathrm{P}_{n}\right\}$. Notice that for any $m$ at most one of the interaction potentials $Q\left(t_{m}\right), Q_{2}\left(t_{m}\right)$ is different from zero. Since $\Delta Q\left(t_{n}\right)=$ $Q\left(t_{n}+\right)-Q\left(t_{n-1}+\right), \Delta Q_{2}\left(t_{n}\right)=Q_{2}\left(t_{n}+\right)-Q_{2}\left(t_{n-1}+\right)$, we obtain

$$
\begin{aligned}
& \sum_{m}\left\{[\bar{a}]_{*} K_{0} Q\left(t_{m}-\right)+\frac{1}{2\left(1-[\bar{a}]_{*}\right)} Q_{2}\left(t_{m}-\right)\right\} \\
& \leq\left\{\sum_{n} K\left(Q\left(t_{n-1}+\right)-Q\left(t_{n}+\right)\right)+K_{2}\left(Q_{2}\left(t_{n-1}+\right)-Q_{2}\left(t_{n}+\right)\right)\right\} \\
& \leq K Q(0+)+K_{2} Q_{2}(0+),
\end{aligned}
$$

which proves (8.23).

9. Decomposition by paths. In this section we introduce in detail the technique of decomposition by paths, [5, 222.

Let $U^{\nu}(x, t)$ be an approximate solution defined for $0 \leq t<T$. Consider a sequence $\mathrm{P}_{0}, \mathrm{P}_{1}, \ldots, \mathrm{P}_{n}$ of interaction points in the plane $(x, t)$, with $\mathrm{P}_{m}=\left(x_{m}, t_{m}\right)$ and $0 \leq t_{0}<$ $t_{1}<\ldots<t_{n}$, such that $\mathrm{P}_{m-1}$ and $\mathrm{P}_{m}$ are connected by a shock wave, for $m=1, \ldots, n$. The type $c_{m}$ of the segment $\mathrm{P}_{m-1} \mathrm{P}_{m}$ is defined for $m \geq 1$ by

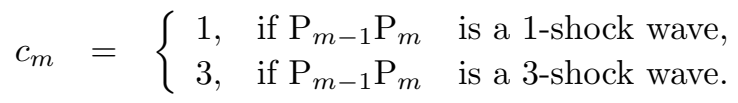

A path $\Gamma$ is a polygonal time-like line, joining the points $\mathrm{P}_{0}, \mathrm{P}_{1}, \ldots, \mathrm{P}_{n}$, whose formation rules are detailed below; see [5]. It is denoted $\Gamma: \mathrm{P}_{0} \rightarrow \mathrm{P}_{1} \rightarrow \cdots \rightarrow \mathrm{P}_{n}$. In order to 
consider also noninteracting shocks in a path $\Gamma$, we allow the last point $\mathrm{P}_{n}$ to be any point on the segment defining the shock and not necessarily an interaction point.

Roughly speaking, the decomposition by paths runs as follows. At any interaction of waves of families 1 or 3 the strength of an outgoing shock is decomposed into a linear and a quadratic part, as in Proposition 5.7 possibly one of them is missing. On the contrary, no decomposition is done for interactions with a 2-wave. Such parts are the strengths of the segment defined by that shock; each couple formed by the segment and one of its strengths gives rise to a path. A path is called primary and denoted $\Gamma^{P}$ if it starts from $t=0$ and all of its segments have strengths obtained either from linear parts of decompositions or from shocks transmitted through an interaction with a 2-wave. Secondary paths, denoted $\Gamma^{S}$, are associated to the quadratic parts of the decomposition; they cannot start from $t=0$.

Primary and secondary paths are constructed in the inductive way below; there we define their generation orders and strengths, which are piecewise constant functions of the segments of the path. Moreover, we define the generation orders of shock, rarefaction and nonphysical waves. For the cases below we refer to Section 5 interactions with 2-waves are enumerated by (i), (ii),..., between 1,3 waves by $(1),(2), \ldots$, and with nonphysical waves by (a), (b),...

- All shock waves issuing from $t=0$ give rise to primary paths which are composed by single segments. More precisely, if $\delta_{i}$ is a shock issuing from $\mathrm{P}_{0} \in\{t=0\}$ and interacting at $\mathrm{P}_{1}=\left(x_{1}, t_{1}\right)$, then the path composed by the segment $\mathrm{P}_{0} \mathrm{P}_{1}$ is a primary path $\Gamma^{P}: \mathrm{P}_{0} \rightarrow \mathrm{P}_{1}$ of generation order 1 and strength $\left|\delta_{i}\right|$. Assign generation order 1 to all discontinuities arising in the approximation of a rarefaction wave issuing from $t=0$.

- Consider the first interaction time; denote by $\mathrm{P}_{1}$ the interaction point and assume that the accurate Riemann solver is used in solving the interaction. Refer to Figure 8 for generation orders assigned to rarefactions; the general definition is given in the next step.

If no shock is involved in the interaction, three cases are possible: both in case (i) and in case (1), two interacting rarefactions, no path is generated; in case (ii) we define a secondary path $\Gamma^{S}: \mathrm{P}_{1} \rightarrow \mathrm{P}_{2}^{\prime}$ of order 2 and strength $h^{\prime \prime}\left|\delta_{2}\right| \cdot\left|\delta_{i}\right|$.

On the contrary, assume that a shock $\delta_{i}$ from $\mathrm{P}_{0} \in\{t=0\}$ interacts at $\mathrm{P}_{1}$ with a (necessarily physical) wave $\delta$. Let $\varepsilon_{i}, \varepsilon_{j}$ be the emitted waves $(i, j \in\{1,3\})$ and $\mathrm{P}_{2}, \mathrm{P}_{2}^{\prime}$ the next interaction points for the waves $\varepsilon_{i}, \varepsilon_{j}$, respectively; we have the following cases.

(iii) We decompose the path $\Gamma^{P}$ into the paths $\Gamma_{1}^{P}$ and $\Gamma_{2}^{P}$ : the path $\Gamma_{1}^{P}$ has generation order 1 and strength $\left|\varepsilon_{i}\right|$; the path $\Gamma_{2}^{P}$ has order 1 and strength $\left|\delta_{i}\right|-\left|\varepsilon_{i}\right|$. The path $\Gamma_{1}^{P}$ is extended to $\mathrm{P}_{2}$ with the generation order and the strength unchanged; the path $\Gamma_{2}^{P}$ stops at $\mathrm{P}_{1}$. A secondary path $\Gamma^{\prime S}: \mathrm{P}_{1} \rightarrow \mathrm{P}_{2}^{\prime}$ is generated, of order 2 and strength $h^{\prime \prime}\left|\delta_{2}\right| \cdot\left|\delta_{i}\right|$.

(iv) The path $\Gamma^{P}$ is extended to $\mathrm{P}_{2}$; the generation order and the strength of $\mathrm{P}_{0} \mathrm{P}_{1}$ are unchanged, the order of $\mathrm{P}_{1} \mathrm{P}_{2}$ is 1 , and the strength is $\left|\delta_{i}\right|$. A secondary path $\Gamma^{\prime S}: \mathrm{P}_{1} \rightarrow \mathrm{P}_{2}$ is generated with order 2 and strength $h^{\prime \prime}\left|\delta_{2}\right| \cdot\left|\delta_{i}\right|$.

(1) If $\delta_{i}$ is a shock, the path $\Gamma^{P}$ is extended to $\mathrm{P}_{2}$, with order and strength unchanged.

(2) Two new paths $\Gamma_{1}^{P}, \Gamma_{2}^{P}$ starting from $\mathrm{P}_{0}$ are defined, and the segment $\mathrm{P}_{0} \mathrm{P}_{1}$ belongs to both of them. The path $\Gamma_{1}^{P}$ has generation order 1 and strength $\left|\varepsilon_{i}\right|=\left|\delta_{i}\right|-\zeta_{i}$ 
in the segment $\mathrm{P}_{0} \mathrm{P}_{1}$; it is extended to $\mathrm{P}_{2}$ where the order of $\mathrm{P}_{1} \mathrm{P}_{2}$ is 1 , and the strength is $\left|\varepsilon_{i}\right|$. The path $\Gamma_{2}^{P}$ has order 1 and strength $\zeta_{i}$ in the segment $\mathrm{P}_{0} \mathrm{P}_{1}$; it is extended to $\mathrm{P}_{2}^{\prime}$, where the order of $\mathrm{P}_{1} \mathrm{P}_{2}^{\prime}$ is 2 , and the strength is $\left|\varepsilon_{j}\right|=h^{\prime} \zeta_{i}$.

(3) The path $\Gamma^{P}$ is extended to $\mathrm{P}_{2}^{\prime}$; the generation order and the strength of $\mathrm{P}_{0} \mathrm{P}_{1}$ are unchanged, the order of $\mathrm{P}_{1} \mathrm{P}_{2}^{\prime}$ is 2 , and the strength is $\left|\varepsilon_{j}\right|=h^{\prime}\left|\delta_{i}\right|$.

(4) The path $\Gamma^{P}$ is extended to $\mathrm{P}_{2}$. The generation order and the strength of $\mathrm{P}_{0} \mathrm{P}_{1}$ are unchanged; the order of $\mathrm{P}_{1} \mathrm{P}_{2}$ is 1 , and the strength is $\left|\delta_{i}\right|$. A secondary path $\Gamma^{S}: \mathrm{P}_{1} \rightarrow \mathrm{P}_{2}$ is generated with order 2 and strength $h^{\prime \prime}\left|\delta_{i} \delta_{i}^{\prime}\right|$. Observe that the shock $\delta_{i}^{\prime}$ belongs to another primary path that is extended to $\mathrm{P}_{1} \rightarrow \mathrm{P}_{2}$ with order 2 and strength $\left|\delta_{i}^{\prime}\right|$.

If the wave $\delta_{i}$ interacts with a physical wave $\delta$ but $\left|\delta_{i} \delta\right|<\rho$, then the simplified Riemann solver is used. Cases (i), (ii) and (1), two interacting rarefactions, do not give rise to paths. In cases (iii) and (iv) a path is extended with the same order; the same happens in case (1) if a shock interacts. When a shock $\delta_{i}$ meets a rarefaction $\delta_{i}^{\prime}$ of the same family, then the path is extended with strength $\left|\delta_{i}\right|-\delta_{i}^{\prime}$ if $\left|\delta_{i}\right|-\delta_{i}^{\prime}>0$; otherwise it stops. If a shock $\delta_{i}$ meets another shock $\delta_{i}^{\prime}$ of the same family, then the path is extended with the same strength.

- At last suppose that all paths have been constructed up to the interaction time $t=t_{n}$. We have thus a collection of paths $\boldsymbol{\Gamma}=\left\{\Gamma_{\ell}\right\}$ in the approximate solution for $t \leq t_{n}$

Let $\gamma$ be a shock wave. The segment associated to $\gamma$ belongs to totally $N$ primary and secondary paths which can be ordered, as far as that segment is concerned, as follows: first the $p$ primary paths for increasing generation order of the segment, then
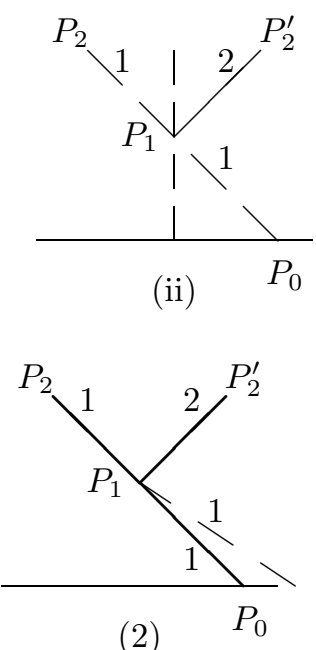
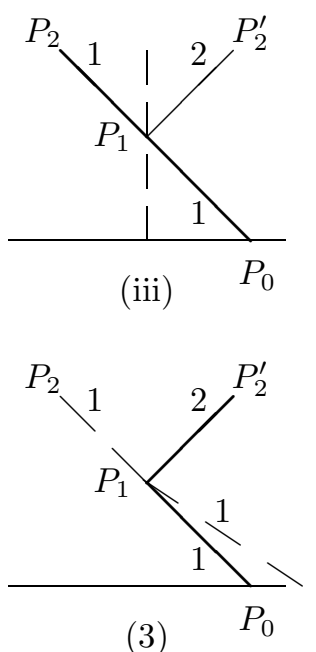
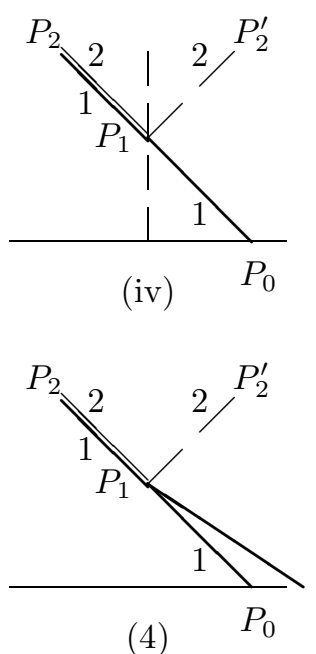

FIG. 8. Interactions of an $i=1$ wave with a 2 -wave (above) and with another 1-wave (below). Primary paths are drawn as thick lines, secondary paths with thin lines, rarefactions and contact discontinuities with broken lines. Numbers denote either generation orders of paths or generation order of the rarefaction waves. 
the $s=N-p$ secondary paths, again for increasing generation order of the segment. That is,

$$
\Gamma_{1}^{P}, \ldots \Gamma_{p}^{P}, \Gamma_{p+1}^{S}, \ldots \Gamma_{N}^{S}, \quad \text { for } \quad k_{1}^{P} \leq \ldots \leq k_{p}^{P}, k_{p+1}^{S} \leq \ldots \leq k_{N}^{S},
$$

where $k_{m}^{P}, k_{m}^{S}$ denote the generation orders of the segment; we drop the indexes $P$ and $S$ in the following. Then, we define the generation order of the shock wave $\gamma$ by

$$
k_{\gamma}=\min _{1 \leq l \leq N}\left\{k_{l}\right\} .
$$

The generation order of rarefaction and nonphysical waves is now introduced; see Figure 9. The next definition applies of course to the special case considered in the previous step.

We first consider rarefactions; recall that by construction of approximate solutions, a rarefaction wave of size $\sigma$ contains $N=[\sigma / \eta]+1$ fronts and each of them has strength $\sigma / N<\eta$.

If a rarefaction wave of order $k$ interacts with a 2 -wave, then the possibly transmitted (reflected) rarefaction has order $k(k+1$, respectively). By Proposition 5.2 it follows that

$$
\left|\varepsilon_{i}-\delta_{i}\right| \leq\left|\varepsilon_{j}\right| \leq \frac{1}{2} \frac{1}{1-[a]_{*}}\left|\delta_{2}\right|\left|\delta_{i}\right| \leq\left|\delta_{i}\right|
$$

since $L_{2}<2\left(1-[\bar{a}]_{*}\right)$ holds as a consequence of (8.1). Then, the outgoing $i$-rarefaction wave will be decomposed into at most two rarefaction fronts of order $k$ so that $\varepsilon_{i}=$ $\varepsilon_{i}^{(1)}+\varepsilon_{i}^{(2)}$, where

$$
\varepsilon_{i}= \begin{cases}\varepsilon_{i}^{(1)} & \text { if } \delta_{i}<\varepsilon_{i}, \\ \delta_{i}+\varepsilon_{i}^{(2)} & \text { if } \varepsilon_{i} \leq \delta_{i} \leq 2 \varepsilon_{i} .\end{cases}
$$

More precisely, the case $\varepsilon_{i}<\delta_{i}$ always occurs in case (i) (and in that case also $\varepsilon_{j}<\delta_{i}$, as in case (iv)); in case (ii) we may have both cases.

When an $i$-rarefaction front interacts with a $j$-wave, with $i, j=1,3, i \neq j$, strengths and orders do not change. If a rarefaction front interacts with a shock of the same family, cancelation occurs and the rarefaction that is possibly generated (case (3)) still consists of a single front. In case (3) the outgoing rarefaction keeps the same order of the interacting one; in case (4) it is assigned order $\max \left\{k, k^{\prime}\right\}+1$, where $k$ and $k^{\prime}$ are the orders of the colliding shocks.

Second, we consider nonphysical waves. If a wave of order $k$ interacts with a 2 -wave, then the order of the outgoing nonphysical wave is $k+1$. If two waves of the same family 1 or 3 interact and a nonphysical wave is emitted, then it is assigned order $\max \left\{k, k^{\prime}\right\}+1$, where $k, k^{\prime}$ are the orders of interacting shocks (possibly one of them is missing).

We now conclude the definition of decomposition by paths. Consider a path $\Gamma: \mathrm{P}_{0} \rightarrow$ $\mathrm{P}_{1} \rightarrow \cdots \rightarrow \mathrm{P}_{n}$ to $t=t_{n}$ and $\delta_{i}$ a shock connecting $\mathrm{P}_{n-1}$ to $\mathrm{P}_{n}$. We assume that the segment $\mathrm{P}_{n-1} \mathrm{P}_{n}$ is contained in paths $\Gamma_{l}$ of strengths $\left|\delta_{l}\right|, 1 \leq l \leq N$, so that, as in (9.1),

$$
\left|\delta_{i}\right|=\sum_{l=1}^{p}\left|\delta_{l}\right|+\sum_{l=p+1}^{N}\left|\delta_{l}\right| \doteq\left|\delta_{i}^{P}\right|+\left|\delta_{i}^{S}\right| .
$$

Above we omitted the dependence on $i$ in the strengths $\left|\delta_{l}\right|$. As in the previous step, assume that $\delta_{i}$ interacts at $\mathrm{P}_{n}$ with a physical wave $\delta$ and $\left|\delta_{i} \delta\right| \geq \rho$. Let $\varepsilon_{i}$, $\varepsilon_{j}$ be 


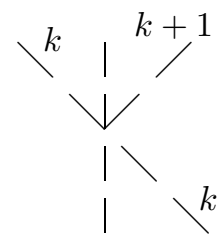

(i)

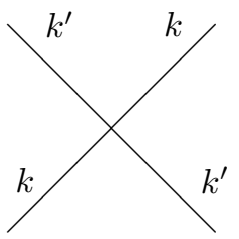

(1)

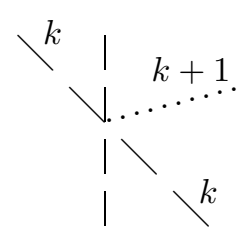

(a)

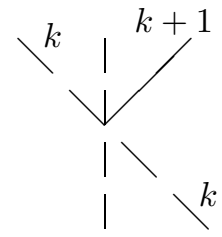

(ii)

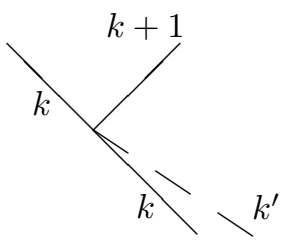

$(2)$

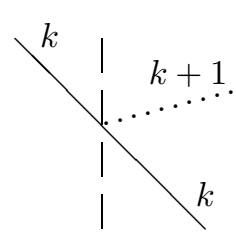

(b)

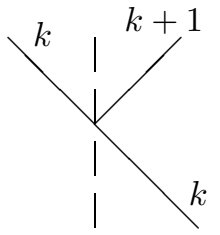

(iii)

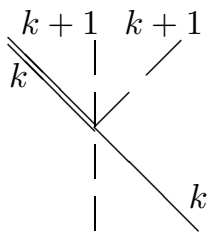

(iv)

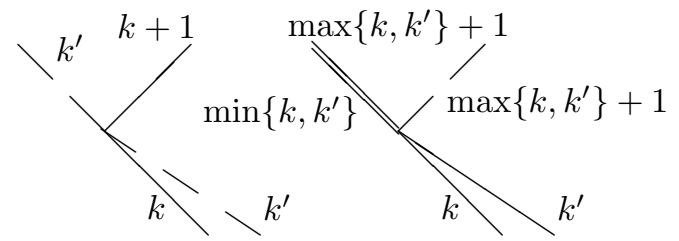

(3)

$(4)$

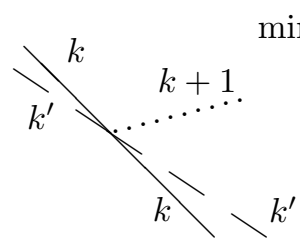

(c)

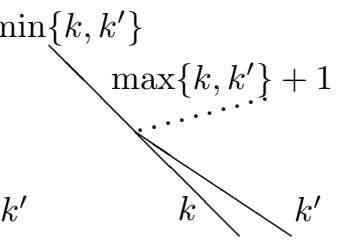

(d)

FIG. 9. Orders of waves. Shocks are represented with solid lines, rarefactions and contact discontinuities with broken lines, nonphysical waves with dotted lines. Secondary shocks (cases (iv) and (4)) are drawn slightly above primary shocks.

the emitted waves $(i, j \in\{1,3\})$ and $\mathrm{P}_{n+1}, \mathrm{P}_{n+1}^{\prime}$ the next interaction points for $\varepsilon_{i}, \varepsilon_{j}$, respectively. Cases (i) and (1), two interacting rarefactions, define no path; in case (ii) if a rarefaction of order $k$ interacts, a path $\Gamma^{\prime S}: \mathrm{P}_{n} \rightarrow \mathrm{P}_{n+1}^{\prime}$ is generated with order $k+1$ and strength $h^{\prime \prime}\left|\delta_{2}\right| \cdot\left|\delta_{i}\right|$. In case (1), when a shock is interacting, all paths $\Gamma_{l}$ extend to $\mathrm{P}_{n+1}$ with orders and strengths unchanged. We are left to the following cases.

(iii) There exist $1 \leq r \leq N$ and $\bar{\delta}_{r}$ with $0 \leq\left|\bar{\delta}_{r}\right|<\left|\delta_{r}\right|$ such that $\left|\delta_{i}\right|-\left|\varepsilon_{i}\right|=\left|\bar{\delta}_{r}\right|+$ $\sum_{l=r+1}^{N}\left|\delta_{l}\right|$; see Figure 10. We split the whole path $\Gamma_{r}$ into two paths $\Gamma_{r}^{(1)}$ and $\Gamma_{r}^{(2)}$ so that the orders of the whole paths are not changed while the absolute values $\alpha_{r}^{(1)}, \alpha_{r}^{(2)}$ of their strengths are decomposed according to the proportion

$$
\alpha_{r}^{(2)}: \alpha_{r}^{(1)}=\left|\bar{\delta}_{r}\right|:\left(\left|\delta_{r}\right|-\left|\bar{\delta}_{r}\right|\right) .
$$

We extend every $\Gamma_{l}$ for $1 \leq l \leq r-1$ and $\Gamma_{r}^{(1)}$ up to $\mathrm{P}_{n+1}$ with orders and strengths unchanged; primary paths remain primary and likewise for secondary paths. The paths $\Gamma_{r}^{(2)}$ and $\Gamma_{l}$ for $r+1 \leq l \leq N$ stop. A secondary path $\Gamma^{\prime S}: \mathrm{P}_{n} \rightarrow \mathrm{P}_{n+1}^{\prime}$ is generated with order $k_{\delta_{i}}+1$ and strength $h^{\prime \prime}\left|\delta_{2}\right| \cdot\left|\delta_{i}\right|$. Here $k_{\delta_{i}}$ is the generation order of the shock; see (9.2). The previous step of this case is obtained with $r=N=1$, $\left|\delta_{i}\right|-\left|\varepsilon_{i}\right|=\left|\bar{\delta}_{r}\right|=\alpha_{r}^{(2)}$. 
(iv) All paths $\Gamma_{l}, 1 \leq l \leq N$, are extended to $\mathrm{P}_{n+1}$ with the same orders and strengths. A secondary path $\Gamma^{\prime \prime S}: \mathrm{P}_{n} \rightarrow \mathrm{P}_{n+1}$ is generated with order $k_{\delta_{i}}+1$ and strength $h^{\prime \prime}\left|\delta_{2}\right| \cdot\left|\delta_{i}\right|$.

(2) We proceed as in case (iii): there exist $1 \leq r \leq N$ and $\bar{\delta}_{r}$ with $0 \leq\left|\bar{\delta}_{r}\right|<\left|\delta_{r}\right|$ such that $\zeta_{i}=\left|\bar{\delta}_{r}\right|+\sum_{l=r+1}^{N}\left|\delta_{l}\right|$. We extend every $\Gamma_{l}$ for $1 \leq l \leq r-1$ to $\mathrm{P}_{n+1}$ with orders and strengths unchanged. Primary paths remain primary and so do secondary paths. Then we split $\Gamma_{r}$ into $\Gamma_{r}^{(1)}$ and $\Gamma_{r}^{(2)}$ so that the orders of the whole paths are not changed while the absolute values $\alpha_{r}^{(1)}, \alpha_{r}^{(2)}$ of their strengths are decomposed according to (9.6). We extend $\Gamma_{r}^{(1)}$ to $\mathrm{P}_{n+1}$ with order and strength unchanged. Let $k_{r}$ be the order of $\Gamma_{r}$ in $\mathrm{P}_{n-1} \mathrm{P}_{n}$; then we extend $\Gamma_{r}^{(2)}$ to $\mathrm{P}_{n+1}^{\prime}$ with order $k_{r}+1$ and strength $h^{\prime}\left|\bar{\delta}_{r}\right|$. At last we extend the paths $\Gamma_{l}$ for $l \geq r+1$ to $\mathrm{P}_{n+1}^{\prime}$ with order $k_{l}+1$ and strength $h^{\prime}\left|\delta_{l}\right|$. The previous step of this case is obtained with $r=N=1$, $\zeta_{i}=\left|\bar{\delta}_{r}\right|=\alpha_{r}^{(2)}$.

(3) The paths $\Gamma_{l}, 1 \leq l \leq N$, are extended to $\mathrm{P}_{n+1}^{\prime}$ leaving unchanged the orders and strengths of their segments until $\mathrm{P}_{n}$; the order of $\mathrm{P}_{n} \mathrm{P}_{n+1}^{\prime}$ is $k_{\delta_{i}}+1$, and the strength is $\left|\varepsilon_{j}\right|=h^{\prime}\left|\delta_{i}\right|$.

(4) Suppose that the shock $\delta_{i}^{\prime}: \mathrm{P}_{n-1}^{\prime} \mathrm{P}_{n}$ interacting at $\mathrm{P}_{n}$ is contained in the paths $\Gamma_{l}^{\prime}$ of strengths $\left|\delta_{l}^{\prime}\right|, 1 \leq l \leq N^{\prime}$. All paths $\Gamma_{l}, 1 \leq l \leq N$, and $\Gamma_{l}^{\prime}, 1 \leq l \leq N^{\prime}$, are extended to $\mathrm{P}_{n+1}$ with their orders and strengths unchanged. A new secondary path $\Gamma^{\prime \prime S}: \mathrm{P}_{n} \rightarrow \mathrm{P}_{n+1}$ is generated with order $\max \left\{k_{\delta_{i}}, k_{\delta_{i}^{\prime}}\right\}+1$ and strength $h^{\prime \prime}\left|\delta_{i} \delta_{i}^{\prime}\right|$.

The case when $\delta_{i}$ interacts with a physical wave $\delta$ and $\left|\delta_{i} \delta\right|<\rho$ is dealt with as in the previous step. At last, in the interaction of a physical wave with a nonphysical wave a path is extended with the same order.

This concludes the definitions of paths. Notice that the generation orders of rarefactions differ from the ones defined in [7]. Moreover, consider the generation orders of shocks, defined in (9.2) and displayed in Figure 9. In case (iv), for instance, the incoming shock has as order $k$ the minimum of the orders $k_{l}$ of its segments; the transmitted shock belong to many paths, some of which have order $k_{l}$ while the others (secondary) have order $k_{l}+1$. Then the order of the transmitted shock is $k$. The same situation occurs in cases (4) and (d).

In this way, a collection of primary paths $\boldsymbol{\Gamma}^{P}=\left\{\Gamma_{\ell}^{P}\right\}$ and secondary paths $\boldsymbol{\Gamma}^{S}=\left\{\Gamma_{\ell}^{S}\right\}$ is defined up to the next interaction time $t_{n+1}$ and hence as long as the approximate solution exists; denote $\boldsymbol{\Gamma}=\boldsymbol{\Gamma}^{P} \cup \boldsymbol{\Gamma}^{S}$. We now make some comments.

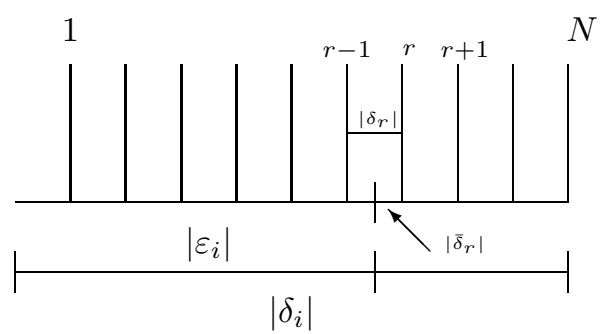

FIG. 10. Order of paths. 
- Let $\Gamma: \mathrm{P}_{0} \rightarrow \mathrm{P}_{1} \rightarrow \cdots \rightarrow \mathrm{P}_{n}$ be a path and $c_{m}, k_{m}, \alpha_{m}$, the type, the generation order and the absolute value of the strength of the segment $\mathrm{P}_{m-1} \mathrm{P}_{m}$ in $\Gamma$, respectively. The sequences $c_{\Gamma}=\left\{c_{m}\right\}, k_{\Gamma}=\left\{k_{m}\right\}$ and $\alpha_{\Gamma}=\left\{\alpha_{m}\right\}$ are called the type, the generation order and the strength (with a slight abuse of terminology) of $\Gamma$, respectively. We may consider the path $\Gamma$ as a Lipschitz curve $x=\Gamma(t)$; then for all $t$ different from interaction times we may think of the type, order and strength of $\Gamma$ as piecewise constant functions of $t$. In particular, the generation order increases along a path; the strength decreases apart from case (iv). If $\Gamma$ is extended to $\Gamma^{\prime}: \mathrm{P}_{0} \rightarrow \mathrm{P}_{1} \rightarrow \cdots \rightarrow \mathrm{P}_{n} \rightarrow \mathrm{P}_{n+1}$, then neither the type nor the generation order change, i.e., $c_{\Gamma}(t)=c_{\Gamma^{\prime}}(t)$ and $k_{\Gamma}(t)=k_{\Gamma^{\prime}}(t)$ for $t \leq t_{n}$; on the contrary the strength may decrease, i.e., $\alpha_{\Gamma}(t) \geq \alpha_{\Gamma^{\prime}}(t)$ for $t \leq t_{n}$, as is clear from cases (iii) and (2).

- The generation order $k_{m}$ of each segment $\mathrm{P}_{m-1} \mathrm{P}_{m}$ in a fixed path $\Gamma$ satisfies

$$
k_{m}=\left\{\begin{array}{ll}
k_{m-1}, & \text { if } \quad c_{m}=c_{m-1} \\
k_{m-1}+1, & \text { if } \quad c_{m} \neq c_{m-1},
\end{array} \quad \text { for } m \geq 2 .\right.
$$

The number $k_{m}$ depends on the path containing the segment. Indeed, consider case (4), Figure 8 the segment $\mathrm{P}_{1} \mathrm{P}_{2}$ has order 1 in the path $\mathrm{P}_{0} \rightarrow \mathrm{P}_{1} \rightarrow \mathrm{P}_{2}$ but order 2 in the secondary path $\mathrm{P}_{1} \rightarrow \mathrm{P}_{2}$. Notice that the generation order of any secondary path is at least 2 .

Observe that (9.7) excludes the possibility of mixed paths formed by primary and secondary segments. Indeed, consider for instance again case (4); the polygonal line joining $\mathrm{P}_{0}, \mathrm{P}_{1}$ and $\mathrm{P}_{2}$ determines a unique primary path since the path $\mathrm{P}_{0} \rightarrow \mathrm{P}_{1}$ cannot be extended to $\mathrm{P}_{2}$ through the secondary segment.

Moreover, consider the case of two shocks of different families connecting for instance $\mathrm{P}_{0}$, resp. $\mathrm{P}_{0}^{\prime}$, with $\mathrm{P}_{1}$ and denote by $\mathrm{P}_{2}$, resp. $\mathrm{P}_{2}^{\prime}$, the next points of interactions in the same directions. Remark that by construction (case (1)) both $\mathrm{P}_{0} \rightarrow \mathrm{P}_{1} \rightarrow \mathrm{P}_{2}$ and $\mathrm{P}_{0}^{\prime} \rightarrow \mathrm{P}_{1} \rightarrow \mathrm{P}_{2}^{\prime}$ are parts of paths, but not $\mathrm{P}_{0} \rightarrow \mathrm{P}_{1} \rightarrow \mathrm{P}_{2}^{\prime}$.

- Consider a shock $\gamma$ at $t$ whose segment belongs to $N$ paths as in (9.1); in general, the number $N$ depends on $t_{n}$. Indeed, consider for instance case (2) above, for the shock connecting $P_{0}$ and $P_{1}$ : if the path is constructed until $t=t_{1}$, then $N=1$; if it is constructed until $t=t_{2}$, then $N=2$ (the shock has been decomposed). However, the total strength of the shock is conserved through the decomposition procedure, and it is the sum of the strengths of the segment associated to the shock.

Since the generation order of a path remains constant when a path is extended, then the generation order $k_{\gamma}$ of the shock does not depend on $t_{n}$ (nor on $t$, in spite of the fact that $N$ does). The definition of $k_{\gamma}$ given in (9.2) differs from that in [7]: in some cases $k_{\gamma}$ can be smaller (for instance in case (ii)); in other cases it can be larger (cases (iv) and (4)).

- A path may stop. This may happen either in case (iii) or when a shock and a rarefaction (whose strength is larger than or equal to that of the shock) interact and the simplified Riemann solver is used.

10. Estimates along paths. A simple consequence of the definition of paths of Section 9 is the following. 
Lemma 10.1. If two paths $\Gamma^{(1)}$ and $\Gamma^{(2)}$ start from the same point and have the same type, then they coincide.

Proof. By contradiction let $\mathrm{P}_{*}$ be the first point from which point on they do not coincide. Let $c^{(1)}, c^{(2)}$ be the types of $\Gamma^{(1)}, \Gamma^{(2)}$. Observe that the paths are either both primary or both secondary.

Assume $\mathrm{P}_{*} \in\{t=0\}$. Therefore both paths are primary; since they start from the same point and differ, then $c^{(1)} \neq c^{(2)}$. This is a contradiction.

Assume $\mathrm{P}_{*} \notin\{t=0\}$. Two primary paths have a segment in common and then branch only in case (2), but in that case the types after the branching are different, a contradiction. If they are secondary, by construction they cannot start from $\mathrm{P}_{*}$, since a single secondary path is generated at a time. Then they were defined before the point $\mathrm{P}_{*}$ and we argue as in the case that they are both primary.

Lemma 10.2. Consider any approximate solution valued in a bounded domain and any path $\Gamma: \mathrm{P}_{0} \rightarrow \mathrm{P}_{1} \rightarrow \cdots \rightarrow \mathrm{P}_{n}$; assume (5.22). Let $k_{m}$ be the generation order and $\alpha_{m}$ the strength of the segment $\mathrm{P}_{m-1} \mathrm{P}_{m}$ in $\Gamma$. Then

$$
\begin{aligned}
& k_{m+1}=k_{m} \\
& k_{m+1}=k_{m}+1 \quad \Rightarrow \quad \alpha_{m+1}=\alpha_{m} \\
& \alpha_{m+1} \leq c \cdot \alpha_{m}
\end{aligned}
$$

Proof. We have $k_{m+1}=k_{m}$ in cases (iii), (iv) and (1) to (4). By construction, in cases (iii) and (1) to (4) the strengths are equal. On the other hand $k_{m+1}=k_{m}+1$ may occur only in cases (2) and (3), where $h^{\prime} \leq c$.

We clearly have the following result by the above lemma.

Lemma 10.3. Assume (5.22). For any approximate solution valued in a bounded domain and $t$ not an interaction time we have:

(i) for any $\Gamma \in \Gamma$ and $0 \leq t^{\prime} \leq t$,

$$
\alpha_{\Gamma}(t) \leq c^{k_{\Gamma}(t)-k_{\Gamma}\left(t^{\prime}\right)} \alpha_{\Gamma}\left(t^{\prime}\right)
$$

(ii) $L(t)=\sum_{\Gamma \in \Gamma} \alpha_{\Gamma}(t)$.

For $\Gamma \in \boldsymbol{\Gamma}$ denote by $t_{0, \Gamma}, P_{0, \Gamma}$ the time, resp. the point, at which $\Gamma$ is generated. Then from (10.2),

$$
\alpha_{\Gamma}(t) \leq c^{k_{\Gamma}(t)-k_{\Gamma}\left(t_{0, \Gamma}\right)} \alpha_{\Gamma}\left(t_{0, \Gamma}\right)
$$

Consider any $t$ not of interaction; for $k=1,2, \ldots$ denote the total amount of the strengths at time $t$ of all primary paths whose generation order at time $t$ is $k$ (resp., larger than $k$ ) by

$$
L_{k}^{P}(t)=\sum_{\Gamma \in \Gamma^{P}, k_{\Gamma}(t)=k} \alpha_{\Gamma}(t), \quad V_{k}^{P}(t)=\sum_{l \geq k} L_{l}^{P}(t)
$$


Proposition 10.4. Assume (5.22). For every approximate solution valued in a bounded domain we have, for $k \geq 1$,

$$
\begin{aligned}
L_{k}^{P}(t) & \leq c^{k-1} L(0), \\
V_{k}^{P}(t) & \leq L(0) \sum_{l \geq k} c^{l-1}=\frac{c^{k-1}}{1-c} L(0) .
\end{aligned}
$$

Proof. If $\Gamma \in \Gamma^{P}$, then $t_{0, \Gamma}=0$; since $k_{\Gamma}(0)=1$ we deduce $\alpha_{\Gamma}(t) \leq c^{k_{\Gamma}(t)-1} \alpha_{\Gamma}(0)$ by (10.3). Then for any $k \geq 1$,

$$
\sum_{\substack{\Gamma \in \boldsymbol{\Gamma}^{P} \\ k_{\Gamma}(t)=k}} \alpha_{\Gamma}(t) \leq c^{k-1} \sum_{\Gamma \in \boldsymbol{\Gamma}^{P}} \alpha_{\Gamma}(0)=c^{k-1} L(0) .
$$

Now, we estimate the total amount of rarefaction waves. The amount of rarefactions may increase, in an interaction, either because a transmitted rarefaction has strength larger than the incoming one or because a new rarefaction is generated. Let us denote

$$
d_{*}=e^{\frac{1}{2} \frac{L_{2}}{1-[\bar{a}]_{*}}}, \quad c_{*}=\max \left\{c, \frac{L_{2}}{2}\right\} e^{\frac{1}{2} \frac{L_{2}}{1-[\bar{a}]_{*}}} .
$$

Proposition 10.5. (A) Consider a rarefaction $\delta_{i}$ generating by interaction another rarefaction $\varepsilon_{i}$ of the same family; then $\left|\varepsilon_{i}\right| \leq\left|\delta_{i}\right|+\frac{1}{2} \frac{1}{1-[\bar{a}]_{*}}\left|\delta_{2} \delta_{i}\right|$. The total increase of $\delta_{i}$ because of such interactions is estimated by

$$
d_{*}\left|\delta_{i}\right|
$$

(B) Let $\delta_{i}$ be an $i$-wave which interacts with a 2 - or an $i$-wave and generates a $j$-rarefaction $\varepsilon_{j}, j \neq i$; then $\left|\varepsilon_{j}\right| \leq \max \left\{c, \frac{L_{2}}{2}\right\}\left|\delta_{i}\right|$. Moreover, assume that

$$
\delta_{i} \text { is }\left\{\begin{array}{l}
\bullet \text { either an } i \text {-rarefaction coming from an } i \text {-rarefaction } \delta_{0}, \\
\bullet \text { or an } i \text {-shock coming from an } i \text {-shock } \delta_{0} \text { such that } \\
\text { the path decompositions of } \delta_{i} \text { and } \delta_{0} \text { are the same. }
\end{array}\right.
$$

Then

$$
\left|\varepsilon_{j}\right| \leq c_{*}\left|\delta_{0}\right|
$$

Proof. (A) By the proofs of cases (i), (ii) in Proposition 5.2 and (1), (3) in Proposition 5.4 we deduce that $\varepsilon_{i} \geq \delta_{i}$ only in case (ii). In such a case, one finds the first estimate above by repeating the calculations leading to (5.12) and (5.14). Then (10.8) follows from

$$
\prod_{\beta: 2 \text {-waves }}\left(1+\frac{1}{2} \frac{|\beta|}{1-[\bar{a}]_{*}}\right)\left|\delta_{i}\right| \leq e^{\frac{1}{2} \sum_{\beta} \frac{|\beta|}{1-[\bar{a}]_{*}}}\left|\delta_{i}\right|=e^{\frac{1}{2} \frac{L_{2}}{1-[\bar{a}]_{*}}}\left|\delta_{i}\right| .
$$

(B) A reflected rarefaction may be generated in cases (i), (iv), (4). Then by (5.3) and (5.26) it follows that $\left|\varepsilon_{j}\right| \leq \max \left\{c, \frac{L_{2}}{2}\right\}\left|\delta_{i}\right|$. The following estimates lead immediately to (10.10).

In case (i), by (5.3) we have

$$
\left|\varepsilon_{j}\right| \leq \frac{1}{2}\left|\delta_{2}\right|\left|\delta_{i}\right| \leq \frac{1}{2} L_{2} e^{\frac{1}{2} \frac{L_{2}}{1-[\bar{a}]_{*}}}\left|\delta_{0}\right|
$$


In case (iv), we find by (5.3) and (10.3),

$$
\left|\varepsilon_{j}\right| \leq \frac{1}{2}\left|\delta_{2}\right|\left|\delta_{i}\right| \leq \frac{1}{2} L_{2} e^{\frac{L_{2}}{2}}\left|\delta_{0}\right|
$$

In case (4), suppose that two shock waves $\delta_{i}, \delta_{i}^{\prime}$ of orders $k \geq k^{\prime}$ interact. By (5.18), (5.25) and (10.3) it follows that

$$
\left|\varepsilon_{j}\right| \leq l_{o}\left|\delta_{i} \delta_{i}^{\prime}\right| \leq c\left|\delta_{i}\right| \leq c e^{\frac{L_{2}}{2}}\left|\delta_{0}\right| .
$$

The total amount of rarefactions with generation order 1 is bounded by $d_{*} L^{+}(0)$ by (10.8). When case (B) in Proposition 10.5 occurs, we briefly say that a new rarefaction is generated. An analogous definition holds for secondary fronts (cases (ii)-(iv), (4)).

Proposition 10.6. Suppose that an $i$-wave $\delta_{i}$ interacts with a 2 -wave and generates a $j$-shock wave $\varepsilon_{j}, j \neq i$. Then, under assumption (10.9), we still have (10.10).

Proof. The eventuality in the assumption occurs in cases (ii) and (iii). One argues as in the proof of case (iv), Proposition 10.5.

We now define for $k \geq 2$

$V_{k}^{S R}(t)$ : the total amount at $t$ of the strengths of both secondary paths and rarefactions whose generation orders are greater than or equal to $k$.

In the previous definition the generation orders are those of both secondary paths and rarefactions. Recall that the generation order of a secondary path is at least 2 .

We denote by $k_{\delta_{i}}$ the generation order of a wave $\delta_{i}$. By (9.5) and (10.6) we obtain for $k \geq 2$,

$$
\sum_{\substack{k_{\delta_{i}} \geq k \\ i=1,3}}\left|\delta_{i}\right|=V_{k}^{P}(t)+V_{k}^{S R}(t) \leq \frac{c^{k-1}}{1-c} L(0)+V_{k}^{S R}(t) .
$$

11. Convergence of approximate solutions. First, following [5], we prove that the approximate solution constructed according to the algorithm above is defined for all $0 \leq t<\infty$.

Proposition 11.1. Consider an approximate solution and let $\mathcal{I}$ be the set of its interaction points. Then the accurate Riemann solver is used at most a finite number of times in $\mathcal{I}$.

Proof. Denote $\mathcal{I}=\left\{\mathrm{P}_{m}\right\}$ with $t_{m}<t_{m+1}$ for $m=1,2, \ldots$. We give a proof in four steps.

(I) First, the claim is true for interactions of either two shocks of the same family or a physical wave with a contact discontinuity. Indeed, in these cases either $Q\left(\mathrm{P}_{m}\right) \geq \rho$ or $Q_{2}\left(\mathrm{P}_{m}\right) \geq \rho$ and we conclude the proof by (8.23).

(II) Secondary fronts and new rarefactions are generated only when the accurate Riemann solver is used, i.e., in cases (i)-(iv), (4). Then, the number of secondary fronts 
and new rarefactions is finite by (I). The total amount of the strengths of the secondary fronts is estimated by (8.23) as

$$
\sum_{m}\left\{[\bar{a}]_{*} K_{0} Q\left(\mathrm{P}_{m}\right)+\frac{1}{2\left(1-[a]_{*}\right)} Q_{2}\left(\mathrm{P}_{m}\right)\right\} \leq \mathcal{Q}(0)
$$

while the total amount of the rarefaction waves is bounded as follows because of Remark 7.4

$$
L^{+}(t) \leq L^{+}(0)+\mathcal{Q}(0)
$$

(III) The claim is true for interactions of shock with rarefaction waves. If the waves are of the same family, a reflected shock wave is always produced; if not, the shock is transmitted. Then, in these interactions a path can always be continued.

Let $\mathrm{P}_{m}$ be an interaction point and denote the strength of the interacting shock (rarefaction) wave by $\delta\left(\mathrm{P}_{m}\right)\left(\delta^{\prime}\left(\mathrm{P}_{m}\right)\right)$. Let $\boldsymbol{\Gamma}_{\delta}\left(\mathrm{P}_{m}\right)$ denote the collection of paths composing $\delta\left(\mathrm{P}_{m}\right)$; for $\Gamma \in \boldsymbol{\Gamma}_{\delta}\left(\mathrm{P}_{m}\right)$ we denote $\alpha_{\Gamma}\left(\mathrm{P}_{m}\right)=\alpha_{\Gamma}\left(t_{m}-\right), k_{\Gamma}\left(\mathrm{P}_{m}\right)=k_{\Gamma}\left(t_{m}-\right)$. By (11.2) the total amount of waves generated by these interactions is estimated by

$$
\begin{aligned}
\sum_{\mathrm{P}_{m}}\left|\delta\left(\mathrm{P}_{m}\right) \delta^{\prime}\left(\mathrm{P}_{m}\right)\right| & =\sum_{\mathrm{P}_{m}} \sum_{\Gamma \in \boldsymbol{\Gamma}_{\delta}\left(\mathrm{P}_{m}\right)}\left|\delta^{\prime}\left(\mathrm{P}_{m}\right)\right| \alpha_{\Gamma}\left(\mathrm{P}_{m}\right) \\
& \leq \sum_{\Gamma \in \boldsymbol{\Gamma}} \sum_{\mathrm{P}_{m} \in \Gamma}\left|\delta^{\prime}\left(\mathrm{P}_{m}\right)\right| \alpha_{\Gamma}\left(\mathrm{P}_{m}\right)
\end{aligned}
$$

where we interchanged the order of summation. Consider then for any $\Gamma \in \boldsymbol{\Gamma}$,

$$
\sum_{\mathrm{P}_{m} \in \Gamma}\left|\delta^{\prime}\left(\mathrm{P}_{m}\right)\right| \alpha_{\Gamma}\left(\mathrm{P}_{m}\right)
$$

In performing the summation it may happen that either the strength $\alpha_{\Gamma}$ does not change across $\mathrm{P}_{m}$ or it does. In the first case the generation order of the path does not change (case (1) and, referring to Figure 8, case (2), path $\mathrm{P}_{0} \mathrm{P}_{1} \mathrm{P}_{2}$ ); in the second case it increases by 1 (case (2), path $\mathrm{P}_{0} \mathrm{P}_{1} \mathrm{P}_{2}^{\prime}$ and case (3)). Therefore, say, for $m$ fixed the strength $\alpha_{\Gamma}\left(\mathrm{P}_{\mathrm{m}}\right)$ factors the sum of some interacting rarefactions until a point $\mathrm{P}_{m}^{\prime}$ is reached where the strength changes. Then $\alpha_{\Gamma}\left(\mathrm{P}_{m}^{\prime}\right)$ factors again and so on. Denote by $\left\{\mathrm{P}_{m}^{\prime}\right\}$ a subsequence of $\left\{\mathrm{P}_{m}\right\}$ such that $k_{\Gamma}\left(\mathrm{P}_{m+1}^{\prime}\right)=k_{\Gamma}\left(\mathrm{P}_{m}^{\prime}\right)+1$; the total sum of the above partial sums of rarefactions is estimated by (11.2), and then by (10.3) we obtain

$$
\begin{aligned}
\sum_{\mathrm{P}_{m} \in \Gamma}\left|\delta^{\prime}\left(\mathrm{P}_{m}\right)\right| \alpha_{\Gamma}\left(\mathrm{P}_{m}\right) & \leq\left(L^{+}(0)+\mathcal{Q}(0)\right) \sum_{\mathrm{P}_{m}^{\prime} \in \Gamma} \alpha_{\Gamma}\left(\mathrm{P}_{m}^{\prime}\right) \\
& \leq\left(L^{+}(0)+\mathcal{Q}(0)\right) \frac{1}{1-c} \alpha_{\Gamma}\left(t_{0, \Gamma}\right) .
\end{aligned}
$$

By separating primary from secondary paths we see by (8.23) that

$$
\sum_{\Gamma \in \boldsymbol{\Gamma}} \alpha_{\Gamma}\left(t_{0, \Gamma}\right) \leq L(0)+\mathcal{Q}(0)=F(0) .
$$

From (11.3) we find that

$$
\sum_{\mathrm{P}_{m}}\left|\delta\left(\mathrm{P}_{m}\right) \delta^{\prime}\left(\mathrm{P}_{m}\right)\right| \leq \frac{1}{1-c}\left(L^{+}(0)+\mathcal{Q}(0)\right) \cdot F(0),
$$

and we conclude this case by arguing as in (I). 
(IV) The claim is true for interactions of shock waves of different families. We use the notation as in (III): let $\delta\left(\mathrm{P}_{m}\right), \delta^{\prime}\left(\mathrm{P}_{m}\right)$ be the strengths of the shocks interacting at $\mathrm{P}_{m}$. Let $\boldsymbol{\Gamma}\left(\mathrm{P}_{m}\right)$ be the set of all paths through $\mathrm{P}_{m}$. Then

$$
\begin{aligned}
\sum_{\mathrm{P}_{m}}\left|\delta\left(\mathrm{P}_{m}\right) \delta^{\prime}\left(\mathrm{P}_{m}\right)\right| & \leq \frac{1}{2} \sum_{\Gamma \in \boldsymbol{\Gamma}} \sum_{\mathrm{P}_{m} \in \Gamma} \alpha_{\Gamma}\left(\mathrm{P}_{m}\right) \sum_{\Gamma^{\prime} \in \boldsymbol{\Gamma}\left(\mathrm{P}_{m}\right) \backslash \Gamma} \alpha_{\Gamma^{\prime}}\left(\mathrm{P}_{m}\right) \\
& \leq \frac{1}{2} \sum_{\Gamma \in \boldsymbol{\Gamma}} \sum_{\Gamma^{\prime} \in \boldsymbol{\Gamma} \backslash \Gamma} \sum_{\mathrm{P}_{m} \in \Gamma \cap \Gamma^{\prime}} \alpha_{\Gamma}\left(\mathrm{P}_{m}\right) \alpha_{\Gamma^{\prime}}\left(\mathrm{P}_{m}\right)
\end{aligned}
$$

by interchanging the order of summations. Consider now $\mathrm{P}_{m}, \mathrm{P}_{m}^{\prime} \in \Gamma \cap \Gamma^{\prime}$, with $t_{m}<t_{m}^{\prime}$, and assume that there is no point of $\Gamma \cap \Gamma^{\prime}$ between $\mathrm{P}_{m}$ and $\mathrm{P}_{m}^{\prime}$. Then both $k_{\Gamma}\left(\mathrm{P}_{m}\right)<$ $k_{\Gamma}\left(\mathrm{P}_{m}^{\prime}\right)$ and $k_{\Gamma^{\prime}}\left(\mathrm{P}_{m}\right)<k_{\Gamma^{\prime}}\left(\mathrm{P}_{m}^{\prime}\right)$ hold, so that there is a gain of a $c^{2}$ factor at each new intersection point. Then

$$
\sum_{\mathrm{P}_{m} \in \Gamma \cap \Gamma^{\prime}} \alpha_{\Gamma}\left(\mathrm{P}_{m}\right) \alpha_{\Gamma^{\prime}}\left(\mathrm{P}_{m}\right) \leq \frac{1}{1-c^{2}} \alpha_{\Gamma}\left(\mathrm{P}_{0, \Gamma}\right) \alpha_{\Gamma^{\prime}}\left(\mathrm{P}_{0, \Gamma^{\prime}}\right) .
$$

Therefore by (11.4),

$$
\begin{aligned}
\sum_{\mathrm{P}_{m}}\left|\delta\left(\mathrm{P}_{m}\right) \delta^{\prime}\left(\mathrm{P}_{m}\right)\right| & \leq \frac{1}{2\left(1-c^{2}\right)} \sum_{\Gamma \in \boldsymbol{\Gamma}} \sum_{\Gamma^{\prime} \in \boldsymbol{\Gamma}} \alpha_{\Gamma}\left(\mathrm{P}_{0, \Gamma}\right) \alpha_{\Gamma^{\prime}}\left(\mathrm{P}_{0, \Gamma^{\prime}}\right) \\
& \leq \frac{1}{2\left(1-c^{2}\right)} F(0)^{2} .
\end{aligned}
$$

We conclude again as in (I). The proposition is then completely proved.

Corollary 11.2. The approximate solution is defined for all $0 \leq t<\infty$.

Proof. We prove that the number of interactions remains finite for any time $0<t<\infty$. First, the number of physical fronts is finite because new physical fronts are generated only when the accurate Riemann solver is used. Second, a new nonphysical front is generated by the interaction of two physical fronts and two physical fronts can interact only once; hence also the number of nonphysical fronts is finite. Then, the total number of fronts is finite.

Now, we estimate the total amount of secondary paths and rarefactions in terms of the quadratic functionals. We define for $k=1,2, \ldots$,

$$
\begin{aligned}
Q_{k}(t)=\sum_{\substack{\sigma_{i}<0, \sigma_{i}^{\prime}<0, i=1,3 \\
\max \left\{k_{i}, k_{i}^{\prime}\right\} \geq k}}\left|\sigma_{i} \sigma_{i}^{\prime}\right|, & Q_{2, k}(t)=\sum_{\substack{\left(\sigma_{2}, \sigma_{i}\right) \in \mathcal{A}, i=1,3 \\
k_{i} \geq k}}\left|\sigma_{2}\right| \cdot\left|\sigma_{i}\right|, \\
\mathcal{Q}_{k}(t) & =K Q_{k}(t)+K_{2} Q_{2, k}(t), \\
\widetilde{\mathcal{Q}}_{k}^{ \pm}(t) & =\sum_{0<\tau<t}\left[\Delta \mathcal{Q}_{k}(\tau)\right]_{ \pm},
\end{aligned}
$$

where $k_{i}=k_{\sigma_{i}}$ and $k_{i}^{\prime}=k_{\sigma_{i}^{\prime}}$ are the generation orders of $\sigma_{i}$ and $\sigma_{i}^{\prime}$. Moreover, we also define

$$
\widetilde{L}=\sup _{t>0} L(t-), \quad \widetilde{V}_{k}^{S R}=\sup _{t>0} V_{k}^{S R}(t), \quad \widetilde{\mathcal{Q}}_{k}^{ \pm}=\sum_{\tau>0}\left[\Delta \mathcal{Q}_{k}(\tau)\right]_{ \pm}
$$


where in the last sum $\tau$ runs over all interaction times; this convention is assumed also in the following. We used the notation $[x]_{ \pm}$to denote the positive and negative parts of the number $x$, so that $x=[x]_{+}-[x]_{-}$. Observe that

$$
\widetilde{\mathcal{Q}}_{k}^{+}(t)-\widetilde{\mathcal{Q}}_{k}^{-}(t)=\sum_{0<\tau \leq t}\left\{\left[\Delta \mathcal{Q}_{k}(\tau)\right]_{+}-\left[\Delta \mathcal{Q}_{k}(\tau)\right]_{-}\right\}=\mathcal{Q}_{k}(t) \geq 0
$$

for $k \geq 2$ and then

$$
\widetilde{\mathcal{Q}}_{k}^{-}(t) \leq \widetilde{\mathcal{Q}}_{k}^{+}(t)
$$

Lemma 11.3. Assume that at time $\tau$ a wave $\varepsilon_{*}$ of order $l \geq 2$ is generated, which is either a secondary wave or a reflected rarefaction as in cases (i), (iv). Then

$$
\left|\varepsilon_{*}\right| \leq\left[\Delta \mathcal{Q}_{l-1}(\tau)\right]_{-} .
$$

Proof. First, a secondary wave is generated in cases (ii), (iii), (iv) and (4). Observe that $\Delta \mathcal{Q}_{l-1}(\tau)=\Delta \mathcal{Q}(\tau)$; moreover, because of (8.6), if $\Delta L \geq 0$, then $\Delta L \leq[\Delta \mathcal{Q}]_{-}$. Refer to Propositions 7.1 and 5.7. In case (ii) we have $\Delta L(\tau)=\left|\varepsilon_{j}\right|=\left|\varepsilon_{*}\right|$. In case (iii) we have $\varepsilon_{*}=\varepsilon_{j}$ and $\Delta L(\tau)=-\left|\delta_{i}-\varepsilon_{i}\right|+\left|\varepsilon_{j}\right| \geq 0$, because of (5.17); notice that the estimate for $\Delta L$ in (7.1) was obtained from that for $\left|\varepsilon_{j}\right|$ and then $\left|\varepsilon_{j}\right|+\Delta \mathcal{Q}(\tau) \leq 0$. In case (iv) we have $0 \leq \Delta L=\left|\varepsilon_{i}\right|-\left|\delta_{i}\right|=\left|\delta_{i}-\varepsilon_{i}\right|=\left|\varepsilon_{*}\right|$. In case (4), we have $\Delta L=\left|\varepsilon_{i}\right|-\left|\delta_{i}\right|-\left|\delta_{i}^{\prime}\right|=\left|\varepsilon_{*}\right| \geq 0$. This proves (11.10) in the case that $\varepsilon_{*}$ is a secondary shock.

Second, in the case that $\varepsilon_{*}=\varepsilon_{j}$ is a rarefaction, the proof goes on as follows. In case (i), $\Delta L=\Delta Q=0, \Delta Q_{2}=-\left|\delta_{2} \delta_{i}\right|+\left(\left|\varepsilon_{i}\right|-\left|\delta_{i}\right|\right) L_{2}^{l, r}+\left|\varepsilon_{j}\right| L_{2}^{r, l} \leq 0$; we have to prove that $\varepsilon_{j}+K_{2} \Delta Q_{2} \leq 0$. Since $\varepsilon_{j}=\delta_{2} \delta_{i} / 2$, the above estimate holds if $K_{2}>1 /\left(2-L_{2}\right)$. This last inequality is satisfied because of (8.16) and (8.1). In case (iv), $0<\Delta L(\tau)=$ $\left|\varepsilon_{i}\right|-\left|\delta_{i}\right|=k_{* j}\left|\varepsilon_{j}\right|$. However, we have on $\varepsilon_{j}$ the same estimate given on $\Delta L$ in Proposition 7.1 and then (11.10) holds again.

Proposition 11.4. We have for $k \geq 2$,

$$
V_{k}^{S R}(t) \leq 2 d_{*}\left(\frac{c^{k-1}}{1-c} L(0)+\sum_{h=0}^{k-2} c^{h} \widetilde{\mathcal{Q}}_{k-1-h}^{+}(t)\right) .
$$

Proof. We discuss separately the contribution to $V_{k}^{S R}(t)$ of secondary paths and rarefactions.

(I) Suppose that, at time $\tau<t$, a secondary path of order $l$ is generated with a wave $\varepsilon_{*}$. We claim that this path contributes to $V_{k}^{S R}(t)$, as a path, by less than

$$
\begin{cases}c^{h}\left[\Delta \mathcal{Q}_{k-h-1}(\tau)\right]_{-} & \text {if } l=k-h \leq k, \\ {\left[\Delta \mathcal{Q}_{k-1}(\tau)\right]_{-}} & \text {if } l \geq k .\end{cases}
$$

Indeed, if $l \leq k$, then $l=k-h$ for some $0 \leq h \leq k-2$. Then, the path contributes to $V_{k}^{S R}(t)$, as a path, if it is continued in the time interval $(\tau, t)$ through at least $h$ interactions of types (2) and (3) (and possibly other interactions of types (iii), (iv), (2), (4)). Therefore we obtain the first line of (11.12) by (10.1) and (11.10).

If $l>k$ the secondary path contributes to $V_{k}^{S R}(t)$ of a term less than $\left[\Delta \mathcal{Q}_{l-1}(\tau)\right]_{-}=$ $\left[\Delta \mathcal{Q}_{k-1}(\tau)\right]_{-}$, again by (11.10), and this proves (11.12). 
As a conclusion, the contribution of secondary paths to $V_{k}^{S R}(t)$ is bounded by

$$
\sum_{h=0}^{k-2} \sum_{0<\tau<t}^{\prime} c^{h}\left[\Delta \mathcal{Q}_{k-h-1}(\tau)\right]_{-},
$$

where the second sum is done on interaction times $\tau$ at which a secondary shock is generated.

(II) Now, consider the contribute of rarefactions. In this case we must recall that once a rarefaction is generated (by interactions, because here $k \geq 2$; see Proposition 10.5. case (B)) it can increase its strength of the factor $d_{*}$ (see Proposition 10.5, case $(\mathrm{A})$ ).

Suppose that at time $\tau<t$ a reflected rarefaction wave $\varepsilon_{j}$ of order $l$ is generated as in cases (i), (iv), (4); see Proposition 10.5, case (B). This wave contributes to $V_{k}^{S R}(t)$ if and only if $l \geq k$.

In cases (i) and (iv) by (11.10) we have the estimate

$$
\varepsilon_{j} \leq\left[\Delta \mathcal{Q}_{l-1}(\tau)\right]_{-} \leq\left[\Delta \mathcal{Q}_{k-1}(\tau)\right]_{-} .
$$

In case (4), Lemma 11.3 does not apply and we argue as follows; let $\delta_{i}$ be the incoming shock with higher order, i.e., $l-1$.

If $\delta_{i}$ is primary, we estimate $\varepsilon_{j} \leq c\left|\delta_{i}\right|$ by (5.26); then, because of (10.6), a bound for the total contribution to $V_{k}^{S R}(t)$ of the rarefactions generated in this case is given by

$$
\frac{c^{k-1}}{1-c} L(0)
$$

In the case that $\delta_{i}$ is secondary, we exploit the estimate (11.12) with $k-1$ replacing there $k$, because $l-1 \geq k-1$. Therefore, also taking into account (5.26), the total amount of rarefactions generated in this case is bounded by

$$
c \sum_{h=0}^{k-3} \sum_{0<\tau<t}^{\prime} c^{h}\left[\Delta \mathcal{Q}_{k-h-2}(\tau)\right]_{-}=\sum_{h=1}^{k-2} \sum_{0<\tau<t}{ }^{\prime} c^{h}\left[\Delta \mathcal{Q}_{k-h-1}(\tau)\right]_{-} .
$$

Above, the second sum is performed on interaction times $\tau$ at which a rarefaction is generated. By (11.14) - (11.16) and (10.8) the total contribution of rarefactions to $V_{k}^{S R}(t)$ is less than

$$
d_{*}\left(\frac{c^{k-1}}{1-c} L(0)+\sum_{h=0}^{k-2} \sum_{0<\tau<t}^{\prime} c^{h}\left[\Delta \mathcal{Q}_{k-h-1}(\tau)\right]_{-}\right) .
$$

This concludes item (II).

In conclusion, by (11.13) and (11.17) we obtain

$$
V_{k}^{S R}(t) \leq 2 d_{*}\left(\frac{c^{k-1}}{1-c} L(0)+\sum_{h=0}^{k-2} c^{h} \widetilde{\mathcal{Q}}_{k-h-1}^{-}(t)\right),
$$

whence (11.11) by (11.9).

Next, we study the variations of the quadratic functionals. For $k \geq 1$ let $I_{k}$ denote the set of times where one of the following three interaction patterns occurs:

- $W^{k} C_{2}$ : a wave of order $k$ and a contact discontinuity,

- $S_{i}^{k} R_{i}$ : a shock wave of order $k$ and a rarefaction wave of the same family, 
- $S_{i}^{k_{i}}\left(S_{i}^{\prime}\right)^{k_{i}^{\prime}}$ : two shock waves of the same family having orders $k_{i}, k_{i}^{\prime}$ and such that $\max \left\{k_{i}, k_{i}^{\prime}\right\}=k$.

LEMMA 11.5. We have the following estimates:

$$
\begin{aligned}
& \Delta \mathcal{Q}_{k}(t) \\
& \leq\left\{\begin{array}{l}
K\left\{V_{k}^{S R}(t-)+\frac{c^{k-1}}{1-c} L(0)\right\}\left\{-\Delta \mathcal{Q}(t)+\left|\varepsilon^{*}\right|\right\}, \quad \text { if } t \in I_{1} \cup \cdots \cup I_{k-2}, k \geq 3, \\
\left\{K L(t-)+K_{2} L_{2}\right\}\left\{-\Delta \mathcal{Q}_{k-1}(t)+K_{0}\left|\delta \delta^{\prime}\right|+\left|\varepsilon^{*}\right|\right\}, \quad \text { if } t \in I_{k-1}, k \geq 2, \\
\left\{K L(t-)+K_{2} L_{2}\right\}\left|\varepsilon^{*}\right|, \quad \text { if } t \in I_{k} \cup I_{k+1} \cup \ldots, k \geq 1 .
\end{array}\right.
\end{aligned}
$$

Above, $\delta$ and $\delta^{\prime}$ are the strengths of two shock waves of the same family interacting at time $t$, while $\varepsilon^{*}$ is the strength of the reflected shock wave generated by an $S R$ interaction at time $t$ of two waves of the same family.

Proof. In the following argument, we use Propositions 5.2 and 5.4 several times. Below, $\delta_{i}$ denotes the strength of a shock interacting with a wave of the same family; with a slight abuse of notation, in the case $W C_{2}$ of the proof below (and only there) we denote by $\delta_{i}$ any wave, $i=1,3$. In particular, in the case $S_{i} R_{i}$, the strength $\varepsilon^{*}=\varepsilon_{j}$ satisfies (5.23).

If $t \in I_{1} \cup \cdots \cup I_{k-2}, k \geq 3$, by (10.6) and Proposition 8.4 we have

$$
\begin{aligned}
\Delta Q_{k}(t) & \leq\left\{\begin{array}{cl}
\frac{1}{2} \frac{1}{1-[\bar{a}]_{*}}\left|\delta_{2}\right|\left|\delta_{i}\right|\left(V_{k}^{S R}(t-)+\frac{c^{k-1}}{1-c} L(0)\right) & : W C_{2} \\
\left|\varepsilon^{*}\right|\left(V_{k}^{S R}(t-)+\frac{c^{k-1}}{1-c} L(0)\right) & : S_{i} R_{i} \\
{[\bar{a}]_{*} K_{0}\left|\delta_{i} \delta_{i}^{\prime}\right|\left(V_{k}^{S R}(t-)+\frac{c^{k-1}}{1-c} L(0)\right)} & : S_{i} S_{i}
\end{array}\right. \\
& \leq\left\{V_{k}^{S R}(t-)+\frac{c^{k-1}}{1-c} L(0)\right\}\left\{-K \Delta Q(t)-K_{2} \Delta Q_{2}(t)+\left|\varepsilon^{*}\right|\right\}, \\
\Delta Q_{2, k}(t) & =0 .
\end{aligned}
$$

Hence, the first line in (11.18) follows.

If $t \in I_{k-1}, k \geq 2$, by (5.25) we have

$$
\begin{aligned}
\Delta Q_{k}(t) & \leq\left\{\begin{array}{cl}
\frac{1}{2} \frac{1}{1-[\bar{a}]_{*}}\left|\delta_{2}\right|\left|\delta_{i}\right| L(t-) & : W C_{2} \\
\left|\varepsilon^{*}\right| L(t-) & : S_{i} R_{i} \\
{[\bar{a}]_{*} K_{0}\left|\delta_{i} \delta_{i}^{\prime}\right| L(t-)} & : S_{i} S_{i}
\end{array}\right. \\
& \leq\left\{\begin{array}{cc}
\left.-K \Delta Q_{k-1}(t)-K_{2} \Delta Q_{2, k-1}(t)+\left|\varepsilon^{*}\right|\right\} L(t-), \\
\frac{1}{2} \frac{1}{1-[\bar{a}]_{*}}\left|\delta_{2}\right|\left|\delta_{i}\right| L_{2} & : W C_{2} \\
\left|\varepsilon^{*}\right| L_{2} & : S_{i} R_{i}
\end{array}\right. \\
& \leq\left\{\begin{array}{cc}
Q_{2, k}(t) & \left\{\begin{array}{c}
S_{i} S_{i} \\
{[\bar{a}]_{*} K_{0}\left|\delta_{i} \delta_{i}^{\prime}\right| L_{2}^{l}+K_{0}\left|\delta_{i} \delta_{i}^{\prime}\right| L_{2}^{r} \leq K_{0}\left|\delta_{i} \delta_{i}^{\prime}\right| L_{2}}
\end{array}\right. \\
\left\{-K \Delta Q_{k-1}(t)-K_{2} \Delta Q_{2, k-1}(t)+K_{0}\left|\delta_{i} \delta_{i}^{\prime}\right|+\left|\varepsilon^{*}\right|\right\} L_{2} .
\end{array}\right.
\end{aligned}
$$

Here above, the letters $l$ and $r$ refer to the case $i=1$; in proving the two final estimates we argued as in the proofs of Propositions 7.1, 7.2 and 8.4. Notice the term $[\bar{a}]_{*} K_{0}\left|\delta_{i} \delta_{i}^{\prime}\right| L_{2}^{l}$ 
appearing in the case $S_{i} S_{i}$ of the estimate of $\Delta Q_{2, k}(t)$, which is due to the secondary shock of order $k$ produced by the interaction.

Hence, the second line in (11.18) follows. Obviously, in that estimate, either the term $K_{0}\left|\delta \delta^{\prime}\right|$ or the term $\left|\varepsilon^{*}\right|$ appears; both can be missing.

Finally, if $t \in I_{k} \cup I_{k+1} \cup \ldots, k \geq 1$, then

$$
\begin{aligned}
\Delta Q_{k}(t) & \leq\left\{\begin{array}{cl}
\frac{1}{2} \frac{1}{1-[\bar{a}]_{*}}\left|\delta_{2}\right|\left|\delta_{i}\right| L(t-) & : W C_{2} \\
\left|\varepsilon^{*}\right| L(t-) & : S_{i} R_{i} \\
-\left|\delta_{i} \delta_{i}^{\prime}\right|+[\bar{a}]_{*} K_{0}\left|\delta_{i} \delta_{i}^{\prime}\right| L(t-) & : S_{i} S_{i}
\end{array}\right. \\
& \leq-\left\{1-[\bar{a}]_{*} K_{0} L(t-)\right\}\left|\delta_{i} \delta_{i}^{\prime}\right|+\frac{1}{2} \frac{1}{1-[\bar{a}]_{*}}\left|\delta_{2}\right|\left|\delta_{i}\right| L(t-)+\left|\varepsilon^{*}\right| L(t-), \\
\Delta Q_{2, k}(t) & \leq\left\{\begin{array}{cc}
-\left|\delta_{2}\right|\left|\delta_{i}\right|+\frac{1}{2} \frac{1}{1-[\bar{a}]_{*}}\left|\delta_{2}\right|\left|\delta_{i}\right| L_{2} & : W C_{2} \\
\left|\varepsilon^{*}\right| L_{2} & : S_{i} R_{i}
\end{array}\right. \\
& \leq-\left(1-\frac{1}{2} \frac{1}{1-[\bar{a}]_{*}} L_{2}\right)\left|\delta_{2}\right|\left|\delta_{i}\right|+K_{0}\left|\delta_{i} \delta_{i}^{\prime}\right| L_{2}+\left|\varepsilon^{*}\right| L_{2} .
\end{aligned}
$$

As above, the letters $l$ and $r$ refer to the case $i=1$. Differently than in the previous case, we can now absorb the term $K_{0}\left|\delta_{i} \delta_{i}^{\prime}\right| L_{2}$. Indeed,

$$
\begin{aligned}
\Delta \mathcal{Q}_{k}(t) \leq & -\left\{K\left(1-K_{0}[\bar{a}]_{*} L(t-)\right)-K_{0} K_{2} L_{2}\right\}\left|\delta_{i} \delta_{i}^{\prime}\right| \\
& -\frac{1}{2} \frac{1}{1-[\bar{a}]_{*}}\left\{K_{2}\left(2\left(1-[\bar{a}]_{*}\right)-L_{2}\right)-K L(t-)\right\}\left|\delta_{2}\right|\left|\delta_{i}\right| \\
& +\left|\varepsilon^{*}\right|\left\{K L(t-)+K_{2} L_{2}\right\} .
\end{aligned}
$$

By (8.12) and (8.7), the proof of (11.18) is complete.

Now, we carry out the estimates of the quadratic waves by using Lemma 11.5. We denote

$$
\kappa_{1}=K \widetilde{L}+K_{2} L_{2} .
$$

Proposition 11.6. Fix $k \geq 2$; denote $\underline{I} \doteq I_{1} \cup \cdots \cup I_{k-2}$ and $\bar{I} \doteq I_{k-1} \cup I_{k} \cup \cdots$. Then

$$
\begin{aligned}
\widetilde{\mathcal{Q}}_{k}^{+} \leq \kappa_{1} \widetilde{\mathcal{Q}}_{k-1}^{+}+\frac{K F(0)}{1-c}\left(\frac{c^{k-1}}{1-c} L(0)+\widetilde{V}_{k}^{S R}\right) & \\
& +\kappa_{1}\left(\frac{K_{0} F(0)}{2}+\frac{c}{1-c}\right)\left(c^{k-2} L(0)+\widetilde{V}_{k-1}^{S R}\right) .
\end{aligned}
$$

Proof. Let $\tau$ be an interaction time. By considering the possible signs of $\Delta \mathcal{Q}_{k}(\tau)$, it follows from (11.18) (writing $\delta_{\tau}$ instead of $\delta_{i}$, and so on) that, for $k \geq 2$,

$$
\begin{aligned}
\widetilde{\mathcal{Q}}_{k}^{+} \leq K\left(\widetilde{V}_{k}^{S R}+\frac{c^{k-1}}{1-c} L(0)\right) \sum_{\underline{I}} & \left\{-\Delta \mathcal{Q}(\tau)+\left|\varepsilon_{\tau}^{*}\right|\right\} \\
& +\kappa_{1}\left(K_{0} \sum_{I_{k-1}^{S S}}\left|\delta_{\tau} \delta_{\tau}^{\prime}\right|+\sum_{\bar{I}}\left\{\left[\Delta \mathcal{Q}_{k-1}(\tau)\right]_{-}+\left|\varepsilon_{\tau}^{*}\right|\right\}\right) .
\end{aligned}
$$

The summations above are made on shock waves $\delta_{\tau}, \delta_{\tau}^{\prime}$ of the same family interacting at time $\tau$ and on shock waves $\varepsilon_{\tau}^{*}$ generated through an $S R$ interaction at time $\tau$. We have 
that

$$
\sum_{\underline{I}}\{-\Delta \mathcal{Q}(\tau)\} \leq \mathcal{Q}(0) \quad \text { and } \quad \sum_{\bar{I}}\left[\Delta \mathcal{Q}_{k-1}(\tau)\right]_{-} \leq \sum_{\bar{I}}\left[\Delta \mathcal{Q}_{k-1}(\tau)\right]_{+} \leq \widetilde{\mathcal{Q}}_{k-1}^{+}
$$

by (11.8). Therefore it follows that

$$
\widetilde{\mathcal{Q}}_{k}^{+} \leq K\left(\widetilde{V}_{k}^{S R}+\frac{c^{k-1}}{1-c} L(0)\right)\left(\mathcal{Q}(0)+\sum_{\underline{I}}\left|\varepsilon_{\tau}^{*}\right|\right)+\kappa_{1}\left(\widetilde{\mathcal{Q}}_{k-1}^{+}+K_{0} \sum_{I_{k-1}^{S S}}\left|\delta_{\tau} \delta_{\tau}^{\prime}\right|+\sum_{\bar{I}}\left|\varepsilon_{\tau}^{*}\right|\right)
$$

Now, we estimate the sums appearing in (11.21). We claim that

$$
\begin{aligned}
\sum_{I_{1} \cup \cdots \cup I_{k-2}}\left|\varepsilon_{\tau}^{*}\right| & \leq \frac{c}{1-c}(L(0)+\mathcal{Q}(0)) \\
\sum_{I_{k-1} \cup I_{k} \cup \cdots}\left|\varepsilon_{\tau}^{*}\right| & \leq \frac{c}{1-c}\left(c^{k-2} L(0)+\widetilde{V}_{k-1}^{S R}\right), \\
\sum_{I_{k-1}^{S S}}\left|\delta_{\tau} \delta_{\tau}^{\prime}\right| & \leq \frac{1}{2}(L(0)+\mathcal{Q}(0))\left(c^{k-2} L(0)+\widetilde{V}_{k-1}^{S R}\right) .
\end{aligned}
$$

First, recall that we assumed that at each interaction time there is precisely one interaction; therefore, interaction times $\tau$ correspond uniquely to interaction points $P_{\tau}$. With a slight abuse of notation we understand $\alpha_{\Gamma}$ and $k_{\Gamma}$ as functions of the point $P$ belonging to the path $\Gamma$. Under this notation, then, at an interaction time $\tau$ we have $\alpha_{\Gamma}(\tau)=\alpha_{\Gamma}(\tau+)=\alpha_{\Gamma}\left(P_{\tau}\right)$ and analogously for $k_{\Gamma}$. We define

$$
\mathcal{I}_{h} \doteq\left\{P_{\tau}: \tau \in I_{h}\right\}
$$

and, as above, $\underline{\mathcal{I}} \doteq \mathcal{I}_{1} \cup \cdots \cup \mathcal{I}_{k-2}, \overline{\mathcal{I}} \doteq \mathcal{I}_{k-1} \cup \mathcal{I}_{k} \cup \cdots$. Consider first (11.22). We have

$$
\sum_{\underline{I}}\left|\varepsilon_{\tau}^{*}\right| \leq \sum_{P \in \underline{\mathcal{I}}} \sum_{\Gamma \in \boldsymbol{\Gamma}(P)} \alpha_{\Gamma}(P) \leq \sum_{\Gamma \in \boldsymbol{\Gamma}} \sum_{P \in \Gamma \cap \underline{\mathcal{I}}} \alpha_{\Gamma}(P) \leq \sum_{\Gamma \in \boldsymbol{\Gamma}} \sum_{P \in \Gamma \cap \underline{\underline{\mathcal{I}}}} c^{k_{\Gamma}(P)-k_{\Gamma}\left(P_{0, \Gamma}\right)} \alpha_{\Gamma}\left(P_{0, \Gamma}\right)
$$

by changing the order of summation. In the last estimate we used (10.3). Clearly, the number $m_{\Gamma}$ of points $P \in \Gamma \cap \underline{\mathcal{I}}$ is less than $k-2$; then for every $\Gamma$ we have

$$
\sum_{P \in \Gamma \cap \underline{\mathcal{I}}} c^{k_{\Gamma}(P)-k_{\Gamma}\left(P_{0, \Gamma}\right)} \leq \sum_{j=1}^{m_{\Gamma}} c^{j} \leq \frac{c}{1-c}
$$

Moreover, notice that by (10.4), (10.5) and (11.1) it follows that

$$
\sum_{\Gamma \in \boldsymbol{\Gamma}} \alpha_{\Gamma}\left(t_{0, \Gamma}\right) \leq L(0)+\mathcal{Q}(0)
$$


From the inequalities above we deduce (11.22). The proof of (11.23) is analogous; however, we estimate the term analogous to the right-hand term in (11.25) as follows:

$$
\begin{aligned}
& \sum_{\Gamma \in \boldsymbol{\Gamma}} \sum_{P \in \Gamma \cap \overline{\mathcal{I}}} c^{k_{\Gamma}(P)-k_{\Gamma}\left(P_{0, \Gamma}\right)} \alpha_{\Gamma}\left(P_{0, \Gamma}\right) \\
& \quad \leq\left(\sum_{\Gamma \in \boldsymbol{\Gamma}^{P}}+\sum_{\Gamma \in \boldsymbol{\Gamma}^{S}}\right) \sum_{P \in \Gamma \cap \overline{\mathcal{I}}} c^{k_{\Gamma}(P)-k_{\Gamma}\left(P_{0, \Gamma}\right)} \alpha_{\Gamma}\left(P_{0, \Gamma}\right) \\
& \quad \leq \frac{c}{1-c}\left(c^{k-2} L(0)+\widetilde{V}_{k-1}^{S R}\right) .
\end{aligned}
$$

Here we used (10.4), (10.5) and the notation (11.7); the estimate (11.23) then follows.

At last we prove the estimate (11.24). Under the notation above we have

$$
\begin{aligned}
\sum_{P \in \mathcal{I}_{k-1}^{S S}}\left|\delta(P) \delta^{\prime}(P)\right| & \leq \frac{1}{2} \sum_{\Gamma \in \boldsymbol{\Gamma}} \sum_{P \in \Gamma \cap \mathcal{I}_{k-1}^{S S}} \alpha_{\Gamma}(P) \sum_{\Gamma^{\prime} \in \boldsymbol{\Gamma}(P) \backslash \Gamma^{\prime}} \alpha_{\Gamma^{\prime}}(P) \\
& \leq \frac{1}{2} \sum_{\Gamma \in \boldsymbol{\Gamma}} \sum_{\Gamma^{\prime} \in \boldsymbol{\Gamma} \backslash \Gamma} \sum_{P \in \Gamma \cap \Gamma^{\prime} \cap \mathcal{I}_{k-1}^{S S}} \alpha_{\Gamma}(P) \alpha_{\Gamma^{\prime}}(P) \\
& \leq \frac{1}{2}(L(0)+\mathcal{Q}(0))\left(\sum_{\Gamma \in \Gamma^{P}}+\sum_{\Gamma \in \Gamma^{S}} \sum_{P \in \Gamma \cap \mathcal{I}_{k-1}^{S S}} \alpha_{\Gamma}(P)\right. \\
& \leq \frac{1}{2}(L(0)+\mathcal{Q}(0))\left(c^{k-2} L(0)+\widetilde{V}_{k-1}^{S R}\right),
\end{aligned}
$$

by arguing as in proving (11.26). This proves (11.24). The estimate (11.20) then follows by (11.21)-(11.24).

Now, we write the estimate (11.20) in a simpler way. We denote

$$
\begin{aligned}
b & \doteq 2 d_{*}\left(\frac{K}{1-c}+\frac{K_{0} \kappa_{1}}{2 c}\right) F(0)+\frac{2 d_{*}}{1-c} \kappa_{1} \doteq b_{1}+b_{2} \\
C & \doteq\left\{\frac{c K F(0)}{(1-c)^{2}}\left(1+2 d_{*}\right)+\kappa_{1}\left(\frac{K_{0} F(0)}{2}+\frac{c}{1-c}\right)\left(1+\frac{2 d_{*}}{1-c}\right)\right\} L(0) .
\end{aligned}
$$

COROllary 11.7. For $k \geq 2$ we have

$$
\begin{aligned}
\widetilde{\mathcal{Q}}_{k}^{+} & \leq\left(\widetilde{\mathcal{Q}}_{1}^{+}+\frac{C}{b}\right)(b+c)^{k-1} \\
\widetilde{V}_{k}^{S R} & \leq 2 d_{*}\left(\frac{L(0)}{1-c}+\widetilde{\mathcal{Q}}_{1}^{+}+\frac{C}{b}\right)(b+c)^{k-1} .
\end{aligned}
$$

Proof. First we prove (11.28) by applying (11.11) to (11.20). In the resulting formula we collect all terms containing $L(0)$; the coefficient of the term with $\widetilde{\mathcal{Q}}_{k-1}^{+}$is bounded by $b$ while all the other terms $c^{h} \widetilde{\mathcal{Q}}_{k-2-h}^{+}$, for $0 \leq h \leq k-3$ have just $b$ as coefficient. Then,

$$
\widetilde{\mathcal{Q}}_{k}^{+} \leq b \sum_{h=0}^{k-2} c^{h} \widetilde{\mathcal{Q}}_{k-1-h}^{+}+C c^{k-2} .
$$

Denote $W_{k} \doteq \widetilde{\mathcal{Q}}_{k}^{+}+c \widetilde{\mathcal{Q}}_{k-1}^{+}+\cdots+c^{k-1} \widetilde{\mathcal{Q}}_{1}^{+}$, so that $\widetilde{\mathcal{Q}}_{k}^{+}=W_{k}-c W_{k-1}$. By (11.30) we deduce

$$
W_{k} \leq(b+c) W_{k-1}+C c^{k-2}
$$


and then, by recursion,

$$
W_{k} \leq(b+c)^{k-1} W_{1}+C c^{k-2} \sum_{h=0}^{k-2}\left(\frac{b+c}{c}\right)^{h} \leq(b+c)^{k-1} W_{1}+\frac{C}{b}(b+c)^{k-1} .
$$

This proves (11.28), because $\widetilde{\mathcal{Q}}_{k}^{+} \leq W_{k}$ and $\widetilde{\mathcal{Q}}_{1}^{+}=W_{1}$. At last, (11.29) follows by (11.11) and (11.32) with $k-1$ replacing $k$.

As a consequence of (11.28), if

$$
b+c<1
$$

holds, then $\widetilde{\mathcal{Q}}_{k}^{+} \rightarrow 0$ and $\widetilde{V}_{k}^{S R} \rightarrow 0$ as $k \rightarrow \infty$. Condition (2.6) is just required to prove (11.33).

Proposition 11.8. Assume (2.6) and that $L_{2} L_{\text {tot }}(0)$ is sufficiently small. Then condition (11.33) holds.

Proof. First, observe that, by (8.16), condition (2.6) is equivalent to

$$
2 d_{*} K_{2} L_{2}=2 e^{\frac{1}{2} \frac{L_{2}}{1-[\bar{a}]_{*}}} K_{2} L_{2}<(1-c)^{2} .
$$

On the one hand, condition (11.34) implies the inequality $K_{2} L_{2}<1-c$ that we previously obtained as a consequence of (8.1) and (8.16). Indeed, in the proof of Proposition 8.1. see (8.11), we essentially required the condition

$$
K L(0)+K_{2} L_{2} \leq 1-c .
$$

The condition (11.35) implies that

$$
\begin{aligned}
\mathcal{Q}(0) & \leq K L(0)^{2}+K_{2} L_{2}\left(L(0)+L^{+}(0)\right) \\
& \leq\left(K L(0)+K_{2} L_{2}\right)\left(L(0)+L^{+}(0)\right) \\
& \leq(1-c) L_{\text {tot }}(0)
\end{aligned}
$$

and then

$$
\kappa_{1} \leq K(L(0)+\mathcal{Q}(0))+K_{2} L_{2} \leq K(2-c) L_{\text {tot }}(0)+K_{2} L_{2},
$$

because we have $\widetilde{L} \leq F(0)$ by Corollary 8.3 ,

On the other hand, consider (11.27); the estimate (11.37) and (8.16) imply

$$
b_{2} \leq d_{*} K_{0} \frac{(2-c)^{2}}{1-c}\left(L_{2}+2[\bar{a}]_{*}\right) \cdot L_{\mathrm{tot}}(0)+\frac{2 d_{*}}{1-c} K_{2} L_{2} \doteq b_{2,1}+b_{2,2} .
$$

Now, condition (11.33) also reads $b_{1}+b_{2}<1-c$. By (11.34), we have $b_{2,2}<1-c$. By (6.4) and (11.37), we claim that

$$
b_{1}+b_{2,1}=\mathcal{O}(1) L_{2} L_{\text {tot }}(0) .
$$

Once we prove this claim, the condition (11.33) shall hold if $L_{2} L_{\text {tot }}(0)$ is sufficiently small.

To prove the claim, notice that (11.36) and the expression of the many constants implies that

$$
\begin{aligned}
F(0) & \leq(2-c) L_{\mathrm{tot}}(0) \\
\kappa_{1} & \leq(2-c) L_{2}\left(\frac{2-c}{4[\bar{a}]_{*}} L_{\mathrm{tot}}(0)+\frac{1}{2\left(1-[\bar{a}]_{*}\right)}\right) .
\end{aligned}
$$


We use $L_{2}+2[\bar{a}]_{*} \leq 2 L_{2}$, see (6.4), and make use of the two estimates above to obtain

$$
b_{1}+b_{2,1} \leq \frac{d_{*}(2-c)^{2}}{[\bar{a}]_{*}(1-c)}\left\{1+\frac{1-c}{8 c}\left(\frac{2-c}{2[\bar{a}]_{*}} L_{\mathrm{tot}}(0)+\frac{1}{1-[\bar{a}]_{*}}\right)\right\} L_{2} L_{\mathrm{tot}}(0),
$$

whence (11.38).

REMARK 11.9. We can make precise how small must be the product $\mathrm{WTV}(\bar{a}) \cdot \operatorname{TV}(\bar{v}, \bar{u})$ in order that Theorem 2.1 holds. A first condition comes from the proof of Proposition 11.8. from (11.39) we must require

$$
\begin{aligned}
\frac{d_{*}(2-c)}{2[\bar{a}]_{*}}\left\{1+\frac{1-c}{8 c}\left(\frac{2-c}{2[\bar{a}]_{*}} L_{\text {tot }}(0)+\frac{1}{1-[\bar{a}]_{*}}\right)\right\} L_{2} L_{\text {tot }}(0) & \\
& <\frac{1}{2} \frac{(1-c)^{2}}{2-c}-\langle\bar{a}\rangle \mathrm{e}^{\langle\bar{a}\rangle} .
\end{aligned}
$$

A second condition comes from (8.17), namely

$$
L_{\text {tot }}(0)<\frac{2\left(1-[\bar{a}]_{*}\right)(1-c)-(2-c) L_{2}}{K_{0}(2-c)^{2}\left(1-[\bar{a}]_{*}\right)\left(L_{2}+2[\bar{a}]_{*}\right)} .
$$

By (6.4) we have $L_{2}+2[\bar{a}]_{*} \leq 2 L_{2}$ and then

$$
\frac{2\left(1-[\bar{a}]_{*}\right)(1-c)-(2-c) L_{2}}{K_{0}(2-c)^{2}\left(1-[\bar{a}]_{*}\right)\left(L_{2}+2[\bar{a}]_{*}\right)} \geq 2[\bar{a}]_{*} \frac{2\left(1-[\bar{a}]_{*}\right)(1-c)-(2-c) L_{2}}{(2-c)^{2}\left(1-[\bar{a}]_{*}\right) L_{2}} .
$$

Therefore, if

$$
L_{2} L_{\text {tot }}(0) \leq 2[\bar{a}]_{*} \frac{2\left(1-[\bar{a}]_{*}\right)(1-c)-(2-c) L_{2}}{(2-c)^{2}\left(1-[\bar{a}]_{*}\right)},
$$

in particular if $L_{2} L_{\text {tot }}(0)$ is small, then (11.41) holds. Conditions (11.40) and (11.42) together make precise the smallness of $\mathrm{WTV}(\bar{a}) \cdot \mathrm{TV}(\bar{v}, \bar{u})$.

Finally we show how to choose a constant $M$, as a function of the initial data, in order that (5.22) holds. We define, using the notation introduced in (8.19),

$$
M=2\left|\left(\bar{v}_{+\infty}, \bar{u}_{+\infty}\right)-\left(\bar{v}_{-\infty}, \bar{u}_{-\infty}\right)\right|+\frac{24}{1-[\bar{a}]_{*}} \operatorname{TV}(\bar{v}, \bar{u}) .
$$

The quantity $c=c\left(M, \bar{a}_{*}, \bar{a}^{*}\right)$ is fixed consequently. In turn, this choice fixes the constants $K$ and $K_{2}$ in (8.16) and then the functional $F$ in (6.3). Denote

$$
\bar{r}_{-\infty}=r\left(\bar{v}_{-\infty}, \bar{u}_{-\infty}\right), \quad \bar{s}_{-\infty}=s\left(\bar{v}_{-\infty}, \bar{u}_{-\infty}\right) .
$$

At last, let $S q\left(\left(\bar{r}_{-\infty}, \bar{s}_{-\infty}\right), M\right)$ denote the square in the $(r, s)$ plane whose sides, of length $M$, are parallel to the $r$ or $s$ axes and whose center is the point $\left(\bar{r}_{-\infty}, \bar{s}_{-\infty}\right)$.

Proposition 11.10. Let $M$ be as in (11.43). Then, every wave interaction involves values of $r$ and $s$ lying within the square $S q\left(\left(\bar{r}_{-\infty}, \bar{s}_{-\infty}\right), M\right)$ in the $(r, s)$ plane.

Proof. We obviously have

$$
\left|(\bar{v}(x), \bar{u}(x))-\left(\bar{v}_{-\infty}, \bar{u}_{-\infty}\right)\right| \leq \operatorname{TV}(\bar{v}, \bar{u}) \leq \frac{24}{1-[\bar{a}]_{*}} \mathrm{TV}(\bar{v}, \bar{u}) .
$$

This means both that TV $(\bar{v}, \bar{u}) \leq M$ and that the initial data are confined in the square

$$
S q\left(\left(\bar{r}_{-\infty}, \bar{s}_{-\infty}\right), M\right) .
$$


By (4.4) we deduce

$$
\left|\varepsilon_{1}\right|+\left|\varepsilon_{3}\right| \leq \frac{2}{1-[\bar{a}]_{*}}\left(\left|r_{R}-r_{L}\right|+\left|s_{R}-s_{L}\right|\right)
$$

Therefore the strength of every wave at time $0+$ is less than $\frac{4}{1-[\bar{a}]_{*}} \mathrm{TV}(\bar{v}, \bar{u}) \leq M$. By (11.36) and (11.45) we deduce

$$
F(0+) \leq L(0+)+(1-c) L_{\mathrm{tot}}(0+) \leq \frac{8}{1-[\bar{a}]_{*}} \mathrm{TV}(\bar{v}, \bar{u}) .
$$

By (8.20) and (11.46) it follows that

$$
\begin{aligned}
& \left|\left(v\left(x, t_{1}-\right), u\left(x, t_{1}-\right)\right)-\left(\bar{v}_{-\infty}, \bar{u}_{-\infty}\right)\right| \\
& \quad \leq \operatorname{TV}\left(v\left(\cdot, t_{1}-\right), u\left(\cdot, t_{1}-\right)\right) \\
& \quad \leq 2\left|\left(\bar{v}_{+\infty}, \bar{u}_{+\infty}\right)-\left(\bar{v}_{-\infty}, \bar{u}_{-\infty}\right)\right|+3 F(0+) \\
& \quad \leq 2\left|\left(\bar{v}_{+\infty}, \bar{u}_{+\infty}\right)-\left(\bar{v}_{-\infty}, \bar{u}_{-\infty}\right)\right|+\frac{24}{1-[\bar{a}]_{*}} \operatorname{TV}(\bar{v}, \bar{u}) .
\end{aligned}
$$

By (11.43) we conclude that TV $\left(v\left(x, t_{1}-\right), u\left(x, t_{1}-\right)\right) \leq M$ and, as a consequence, that $\left(v\left(x, t_{1}-\right), u\left(x, t_{1}-\right)\right)$ belongs to $S q\left(\left(\bar{r}_{-\infty}, \bar{s}_{-\infty}\right), M\right)$. We now iterate the procedure. Suppose that

$$
\left|\left(v\left(x, t_{n}-\right), u\left(x, t_{n}-\right)\right)-\left(\bar{v}_{-\infty}, \bar{u}_{-\infty}\right)\right| \leq M
$$

By the same argument as above we conclude that the strength of any interacting wave at $t=t_{n}+$ is less than $M$, and so on. This concludes the proof.

Estimates of physical waves are obtained by (10.6) and (11.29). Now, we shall briefly carry out the estimates of nonphysical waves, relying on [7, [9]. Note that only the simplified Riemann solver generates a nonphysical wave and nonphysical waves do not interact with each other. The strength of a nonphysical wave is defined as in [7.

Proposition 11.11. Let $\varepsilon$ denote the strength of a nonphysical wave. Then

$$
\begin{aligned}
&|\varepsilon| \leq C_{1} \rho, \\
& \sum_{\substack{\varepsilon \in \mathcal{N} \mathcal{P} \\
k_{\varepsilon} \geq k}}|\varepsilon| \leq C_{2} \sup _{t>0}\left\{V_{k}^{P}(t)+V_{k}^{S R}(t)\right\}
\end{aligned}
$$

for some constants $C_{1}$ and $C_{2}$. Moreover, for any given $h>0$, there exists a threshold $\rho_{h}>0$ so that the approximate solution constructed by the front-tracking scheme satisfies

$$
\sum_{\varepsilon \in \mathcal{N} \mathcal{P}}|\varepsilon| \leq h
$$

Proof. The estimate (11.47) comes from the local interaction estimates. Since the amount of nonphysical waves generated at $I_{k}$ is $\mathcal{O}(1) \cdot\left(V_{k}^{P}(t)+V_{k}^{S R}(t)\right)$, we obtain (11.48). 
Let $N_{0}$ be the number of shock waves at $t=0$. Then there exists a polynomial $P$ such that

$$
\begin{aligned}
\sum_{\varepsilon \in \mathcal{N} \mathcal{P}}|\varepsilon| & =\sum_{\substack{\varepsilon \in \mathcal{N} \mathcal{P} \\
k_{\varepsilon} \leq k-1}}|\varepsilon|+\sum_{\substack{\varepsilon \in \mathcal{N} \mathcal{P} \\
k_{\varepsilon} \geq k}}|\varepsilon| \\
& =\mathcal{O}(1) P\left(N_{0}, h^{-1}\right) \rho+\mathcal{O}(1) \sup _{t>0}\left(V_{k}^{P}(t)+V_{k}^{S R}(t)\right) \\
& =\mathcal{O}(1) P\left(N_{0}, h^{-1}\right) \rho+\mathcal{O}(1) c^{k-1}+\mathcal{O}(1)(b+c)^{k-1}
\end{aligned}
$$

because of (10.6) and (11.29). By (11.33) we can choose $k$ such that $\mathcal{O}(1) c^{k-1}+\mathcal{O}(1)(b+$ $c)^{k-1} \leq \frac{h}{2}$ and then $\rho$ so that (11.49) holds.

Now, we show how to choose the parameters introduced in Section 6 .

Proposition 11.10 implies that the sequences $\left\{r^{\nu}\right\}$ and $\left\{s^{\nu}\right\}$ are bounded; then also $\left\{v^{\nu}\right\}$ is uniformly bounded, both from above and away from 0 , because $v=a(\lambda) \exp \left(\frac{r-s}{2 a_{*}}\right)$ by (3.2). As a consequence, both characteristic speeds $e_{1}$ and $e_{3}$ are bounded and we can choose a suitable speed $\hat{s}$ for the nonphysical waves. Next, consider any sequence $\eta=\eta_{\nu}>0$ that vanishes as $\nu \rightarrow \infty$. Proposition 11.11 provides us with a sequence $\rho=\rho_{1 / \nu}$ that makes the total strength of nonphysical waves less than $1 / \nu$.

The proof of Theorem 2.1 is then completed by following the standard arguments in [7, 9].

Appendix A. On the metric defined by the Riemann invariants. In this appendix we provide some technical results on bounds of states by involving the metric introduced first in (3.3).

Let $w_{j}(U), 1 \leq j \leq N$, be independent Riemann invariants defined in the state space $\Omega \subset \mathbb{R}^{N}$. Observe, however, that we merely need that the map $w: \Omega \rightarrow \mathbb{R}^{N}$ is injective. A metric in $\Omega$ is defined by

$$
\left|U_{B}-U_{A}\right|=\max _{1 \leq j \leq N}\left|w_{j}\left(U_{B}\right)-w_{j}\left(U_{A}\right)\right|
$$

As above, with a slight abuse of notation we use the symbol $|\cdot|$ to denote both the metric (in $\mathbb{R}^{N}$ ) and the absolute value (in $\mathbb{R}$ ). Let $\Delta: a=x_{0} \leq x_{1} \leq \cdots \leq x_{n}=b$ be an arbitrary partition of an interval $[a, b]$. For $U(x): \mathbb{R} \rightarrow \Omega$, its total variation is defined by

$$
\mathrm{TV}_{[a, b]} U=\sup _{\Delta} \sum_{1 \leq l \leq n}\left|U\left(x_{l}\right)-U\left(x_{l-1}\right)\right|
$$

Proposition A.1. Consider a function $U: \mathbb{R} \rightarrow \Omega \subset \mathbb{R}^{N}$ and real numbers $a<b$. Then

$$
|U(b)-U(a)| \leq \operatorname{TV}_{[a, b]} U
$$

Proof. Let $\Delta: a=x_{0} \leq x_{1} \leq \cdots \leq x_{n}=b$ be an arbitrary partition of the interval $[a, b]$. By definition we have, for some $j_{0}$, 


$$
\begin{aligned}
|U(b)-U(a)| & =\left|w_{j_{0}}(U(b))-w_{j_{0}}(U(a))\right| \leq \sum_{1 \leq l \leq n}\left|w_{j_{0}}\left(U\left(x_{l}\right)\right)-w_{j_{0}}\left(U\left(x_{l-1}\right)\right)\right| \\
& \leq \sum_{1 \leq l \leq n}\left|U\left(x_{l}\right)-U\left(x_{l-1}\right)\right| \leq \mathrm{TV}_{[a, b]} U .
\end{aligned}
$$

Now, we study the positive and negative variations. We use the notation

$$
[F(x)]_{+}=\max \{F(x), 0\}, \quad[F(x)]_{-}=\max \{-F(x), 0\} .
$$

Lemma A.2. Consider a function $U: \mathbb{R} \rightarrow \Omega \subset \mathbb{R}^{N}$ and a partition $\Delta: a=x_{0} \leq x_{1} \leq$ $\cdots \leq x_{n}=b$ of the interval $[a, b]$. For every $1 \leq j \leq N$ we have

$$
\begin{aligned}
\sum_{1 \leq l \leq n}\left[w_{j}\left(U\left(x_{l}\right)\right)-w_{j}(\right. & \left.\left.U\left(x_{l-1}\right)\right)\right]_{+} \\
& \leq|U(b)-U(a)|+\sum_{1 \leq l \leq n}\left[w_{j}\left(U\left(x_{l}\right)\right)-w_{j}\left(U\left(x_{l-1}\right)\right)\right]_{-} .
\end{aligned}
$$

Proof. Note that for all $1 \leq j \leq N$ we have

$$
\begin{aligned}
& w_{j}(U(b))-w_{j}(U(a)) \\
& \quad=\sum_{1 \leq l \leq N}\left[w_{j}\left(U\left(x_{l}\right)\right)-w_{j}\left(U\left(x_{l-1}\right)\right)\right]_{+}-\sum_{1 \leq l \leq N}\left[w_{j}\left(U\left(x_{l}\right)\right)-w_{j}\left(U\left(x_{l-1}\right)\right)\right]_{-} .
\end{aligned}
$$

As a consequence,

$$
\begin{aligned}
& \sum_{1 \leq l \leq N}\left[w_{j}\left(U\left(x_{l}\right)\right)-w_{j}\left(U\left(x_{l-1}\right)\right)\right]_{+} \\
& \quad=w_{j}(U(b))-w_{j}(U(a))+\sum_{1 \leq l \leq N}\left[w_{j}\left(U\left(x_{l}\right)\right)-w_{j}\left(U\left(x_{l-1}\right)\right)\right]_{-} \\
& \quad \leq\left|w_{j}(U(b))-w_{j}(U(a))\right|+\sum_{1 \leq l \leq N}\left[w_{j}\left(U\left(x_{l}\right)\right)-w_{j}\left(U\left(x_{l-1}\right)\right)\right]_{-} \\
& \quad \leq|U(b)-U(a)|+\sum_{1 \leq l \leq N}\left[w_{j}\left(U\left(x_{l}\right)\right)-w_{j}\left(U\left(x_{l-1}\right)\right)\right]_{-} .
\end{aligned}
$$

From now on we consider the case $N=2$. This is the case, for instance, of isothermal (isentropic) gas dynamics. However, our case also falls into this framework, as far as the component $\tilde{U}=(v, u)$ of $(v, u, \lambda)$ is concerned, if we choose

$$
w_{1}=r(\tilde{U}), \quad w_{2}=s(\tilde{U}) .
$$

In fact, the Riemann invariants $r$ and $s$, referred to $a(0)$, are constants across the jumps of $\lambda$.

Consider a sequence of states

$$
U_{-\infty}=U_{0}, U_{1}, U_{2}, \ldots, U_{n}=U_{\infty}, \quad \text { in } \Omega \subset \mathbb{R}^{2},
$$


connected by $j$-waves $(1 \leq j \leq 2)$ and define

$$
\begin{aligned}
& \mathcal{L}_{j}^{+}=\left\{l: w_{j}\left(U_{l}\right) \geq w_{j}\left(U_{l-1}\right), 1 \leq l \leq n\right\}, \\
& \mathcal{L}_{j}^{-}=\left\{l: w_{j}\left(U_{l}\right)<w_{j}\left(U_{l-1}\right), 1 \leq l \leq n\right\} .
\end{aligned}
$$

Lemma A.3. For any sequence of states as in (A.2) and every $j=1,2$, we have

$$
\sum_{l \in \mathcal{L}_{j}^{+}}\left[w_{j}\left(U_{l}\right)-w_{j}\left(U_{l-1}\right)\right]_{+} \leq\left|U_{\infty}-U_{-\infty}\right|+\sum_{l \in \mathcal{L}_{j}^{-}}\left[w_{j}\left(U_{l}\right)-w_{j}\left(U_{l-1}\right)\right]_{-} .
$$

Proof. If $U_{l-1}$ and $U_{l}$ are connected by a $j$-rarefaction curve, then it follows from Figure 1 that

$$
w_{j}\left(U_{l}\right)>w_{j}\left(U_{l-1}\right), \quad w_{j^{*}}\left(U_{l}\right)>w_{j^{*}}\left(U_{l-1}\right),
$$

where $j^{*}=2$ for $j=1$ and $j^{*}=1$ for $j=2$. Similarly, if $U_{l-1}$ and $U_{l}$ are connected by a $j$-shock curve, then

$$
w_{j}\left(U_{l}\right)<w_{j}\left(U_{l-1}\right), \quad w_{j^{*}}\left(U_{l}\right)<w_{j^{*}}\left(U_{l-1}\right) .
$$

Moreover, for both cases we have

$$
\left|w_{j^{*}}\left(U_{l}\right)-w_{j^{*}}\left(U_{l-1}\right)\right|<\left|w_{j}\left(U_{l}\right)-w_{j}\left(U_{l-1}\right)\right| .
$$

By Lemma A.2, together with the above observation, we obtain (A.3).

We can now prove our main result. First, for a sequence of states as in (A.2), define

$$
\mathcal{L}^{-}=\left\{l ; 1 \leq l \leq n, U_{l-1} \text { and } U_{l} \text { are connected by a shock curve }\right\} .
$$

Theorem A.4. Consider a sequence of states as in A.2 connected by $j$-waves $(j=1,2)$. Then

$$
\sum_{1 \leq l \leq n}\left|U_{l}-U_{l-1}\right| \leq 2\left|U_{\infty}-U_{-\infty}\right|+3 \sum_{l \in \mathcal{L}^{-}}\left|U_{l}-U_{l-1}\right|
$$

Proof. Let us define the index $j(l)$ so that

$$
\left|U_{l}-U_{l-1}\right|=\left|w_{j(l)}\left(U_{l}\right)-w_{j(l)}\left(U_{l-1}\right)\right| .
$$

We find by (A.4) that $j(l)$ is well defined. It follows by Lemma A.3 that

$$
\begin{aligned}
& \sum_{1 \leq l \leq n}\left|U_{l}-U_{l-1}\right|=\sum_{\substack{1 \leq l \leq n \\
j^{\prime}=1,2}}\left|w_{j(l)}\left(U_{l}\right)-w_{j(l)}\left(U_{l-1}\right)\right| \\
& \leq 2\left|U_{\infty}-U_{-\infty}\right|+\sum_{\substack{j^{\prime} \\
l \in \mathcal{L}^{\prime}}}^{+} \sum_{j^{\prime}=1,2}\left[w_{l \in \mathcal{L}_{j^{\prime}}^{-}}\left[w_{j^{\prime}}\left(U_{l}\right)-w_{j^{\prime}}\left(U_{l-1}\right)\right]_{+}+\sum_{j^{\prime}=1,2} \sum_{\substack{j(l)=j^{\prime} \\
l \in \mathcal{L}_{j^{\prime}}^{-}}}\left[w_{j^{\prime}}\left(U_{l-1}\right)\right]_{-}-w_{j^{\prime}}\left(U_{l-1}\right)\right]_{-} \\
& \quad+\sum_{j^{\prime}=1,2} \sum_{\substack{j(l)=j^{\prime} \\
l \in \mathcal{L}_{j^{\prime}}^{-}}}\left[w_{j^{\prime}}\left(U_{l}\right)-w_{j^{\prime}}\left(U_{l-1}\right)\right]_{-} .
\end{aligned}
$$

If $j^{\prime} \neq j(l)$, then $j^{\prime}=j(l)^{*}$. By using (A.4), we have, if $j^{\prime} \neq j(l)$,

$$
\left[w_{j^{\prime}}\left(U_{l}\right)-w_{j^{\prime}}\left(U_{l-1}\right)\right]_{-}<\left[w_{j(l)}\left(U_{l}\right)-w_{j(l)}\left(U_{l-1}\right)\right]_{-} .
$$


Thus

$$
\begin{aligned}
& \left.\sum_{j^{\prime}=1,2} \sum_{\substack{l \in \mathcal{L}_{j^{\prime}}^{-} \\
\left[w_{j^{\prime}}\right.}}\left(U_{l}\right)-w_{j^{\prime}}\left(U_{l-1}\right)\right]_{-} \leq 2 \sum_{\substack { j^{\prime}=1,2 \\
\begin{subarray}{c}{j(l)=j^{\prime} \\
l \in \mathcal{L}_{j^{\prime}}^{-}{ j ^ { \prime } = 1 , 2 \\
\begin{subarray} { c } { j ( l ) = j ^ { \prime } \\
l \in \mathcal { L } _ { j ^ { \prime } } ^ { - } } }\end{subarray}}\left[w_{j^{\prime}}\left(U_{l}\right)-w_{j^{\prime}}\left(U_{l-1}\right)\right]_{-} \\
& =2 \sum_{l \in \mathcal{L}^{-}}\left|U_{l}-U_{l-1}\right| \text {. }
\end{aligned}
$$

This proves the theorem.

\section{REFERENCES}

[1] Debora Amadori and Andrea Corli, A hyperbolic model of multi-phase flow, Hyperbolic Problems: Theory, Numerics, Applications. Proceedings of the $11^{\text {th }}$ Int. Conf. on Hyperbolic Problems (Sylvie Benzoni-Gavage and Denis Serre, eds.), Springer, 2008, pp. 407-414. MR 2549172

[2] _ On a model of multiphase flow, SIAM J. Math. Anal. 40 (2008), no. 1, 134-166. MR2403315

[3] _ Global existence of BV solutions and relaxation limit for a model of multiphase reactive flow, Nonlinear Anal. 72 (2010), no. 5, 2527-2541. MR2577817

[4] Debora Amadori and Graziano Guerra, Global BV solutions and relaxation limit for a system of conservation laws, Proc. Roy. Soc. Edinburgh Sect. A 131 (2001), no. 1, 1-26. MR.1820292 (2002a:35135)

[5] Fumioki Asakura, Wave-front tracking for the equations of isentropic gas dynamics, Quart. Appl. Math. 63 (2005), no. 1, 20-33. MR2126567(2005k:35256)

[6] François Bereux, Eric Bonnetier, and Philippe G. LeFloch, Gas dynamics system: two special cases, SIAM J. Math. Anal. 28 (1997), no. 3, 499-515. MR1443605 (98d:76143)

[7] Alberto Bressan, Hyperbolic systems of conservation laws. The one-dimensional Cauchy problem, Oxford University Press, 2000. MR.1816648 (2002d:35002)

[8] Andrea Corli and Haitao Fan, The Riemann problem for reversible reactive flows with metastability, SIAM J. Appl. Math. 65 (2004/05), no. 2, 426-457. MR2123064(2005j:35149)

[9] Constantine M. Dafermos, Hyperbolic conservation laws in continuum physics, third ed., SpringerVerlag, Berlin, 2010. MR2169977 (2006d:35159)

[10] Haitao Fan, On a model of the dynamics of liquid/vapor phase transitions, SIAM J. Appl. Math. 60 (2000), no. 4, 1270-1301. MR.1760035 (2001b:76092)

[11] L. Gosse, Existence of $L^{\infty}$ entropy solutions for a reacting Euler system, Port. Math. (N.S.) $\mathbf{5 8}$ (2001), no. 4, 473-484. MR1881874 (2003f:76082)

[12] Helge Holden, Nils Henrik Risebro, and Hilde Sande, The solution of the Cauchy problem with large data for a model of a mixture of gases, J. Hyperbolic Differ. Equ. 6 (2009), no. 1, 25-106. MR.2512503 (2010j:35316)

[13] _ Front tracking for a model of immiscible gas flow with large data, BIT 50 (2010), no. 2, 331-376. MR2640017

[14] Randall J. LeVeque, Helen C. Yee, Philip Roe, and Bram van Leer, Model systems for reacting flows, NASA-Ames University Consortium NCA2-185 (1988).

[15] Tai Ping Liu, Initial-boundary value problems for gas dynamics, Arch. Rational Mech. Anal. 64 (1977), no. 2, 137-168. MR0433017 (55:5996)

[16] Solutions in the large for the equations of nonisentropic gas dynamics, Indiana Univ. Math. J. 26 (1977), no. 1, 147-177. MR 0435618 (55:8576)

[17] Yunguang Lu, Hyperbolic conservation laws and the compensated compactness method, Chapman \& Hall/CRC, Boca Raton, FL, 2003. MR.1936672 (2004b:35223)

[18] Takaaki Nishida, Global solution for an initial boundary value problem of a quasilinear hyperbolic system, Proc. Japan Acad. 44 (1968), 642-646. MR0236526 (38:4821)

[19] Takaaki Nishida and Joel A. Smoller, Solutions in the large for some nonlinear hyperbolic conservation laws, Comm. Pure Appl. Math. 26 (1973), 183-200. MR0330789 (48:9126)

[20] Yue-Jun Peng, Solutions faibles globales pour un modèle d'écoulements diphasiques, Ann. Scuola Norm. Sup. Pisa Cl. Sci. (4) 21 (1994), no. 4, 523-540. MR.1318771 (96a:35108)

[21] Joel Smoller, Shock waves and reaction-diffusion equations, second ed., Springer-Verlag, New York, 1994. MR.1301779 (95g:35002) 
[22] B. Temple and R. Young, The large time stability of sound waves, Comm. Math. Phys. 179 (1996), no. 2, 417-466. MR:1400747 (97f:35132) 